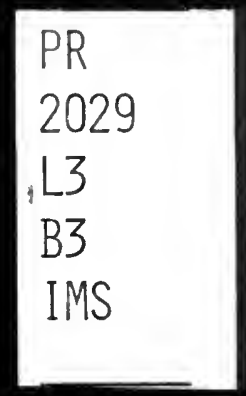




\section{Digitized by the Internet Archive in 2007 with funding from Microsoft Corporation}




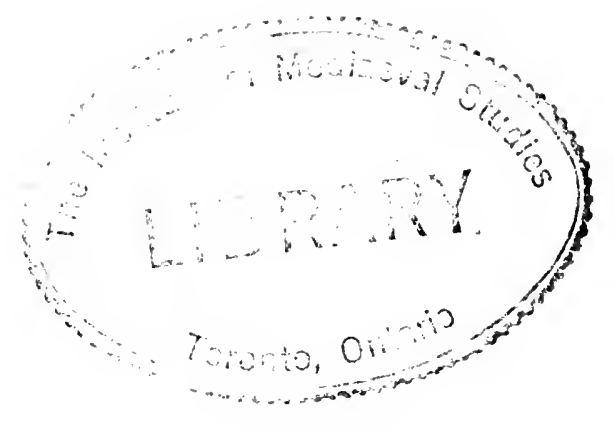







\title{
AN ANGLO-FRENCH LIFE OF SAINT OSITH
}

BT

\author{
A. T. BAKER
}

[FNOM THE MODERN LANGUAGE REVIEW, YoLS, VI AND VII.]

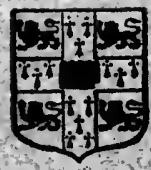

CAMBRIDGE

AT THE UNIVERSITY PRESS 


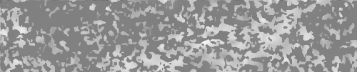

3
3

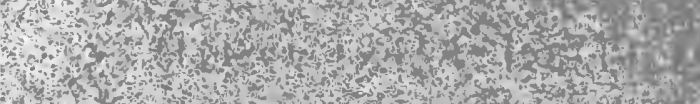
the

Q

ifition

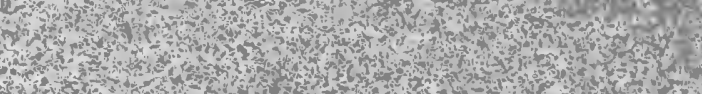

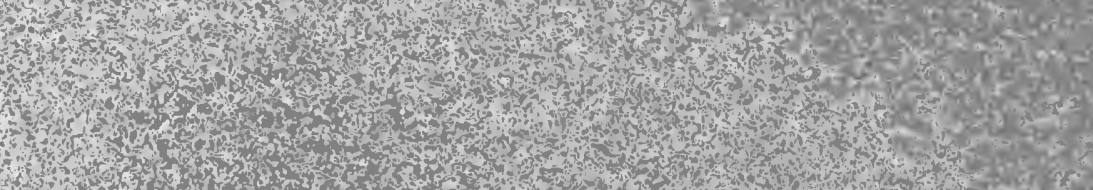

1.7.

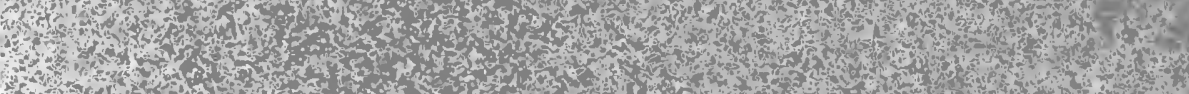

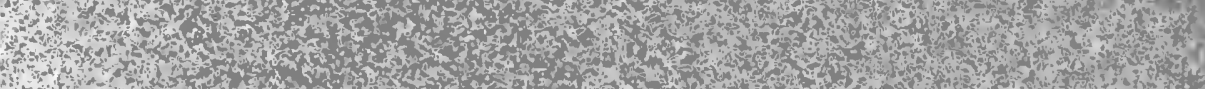

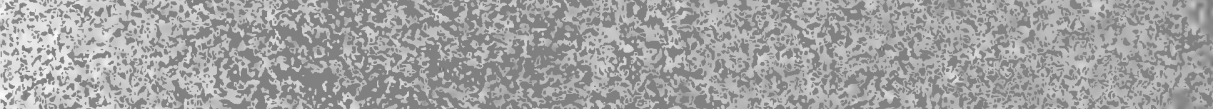

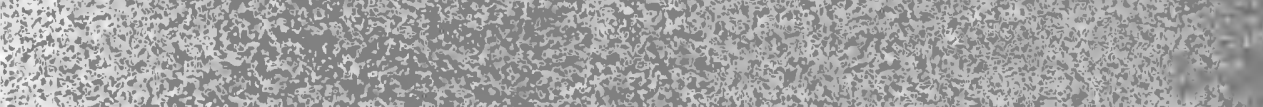

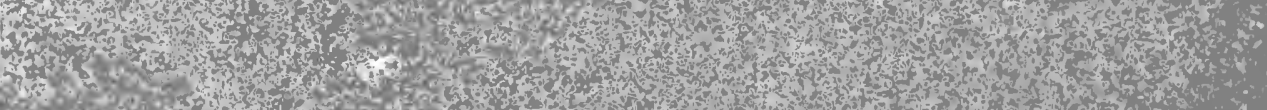

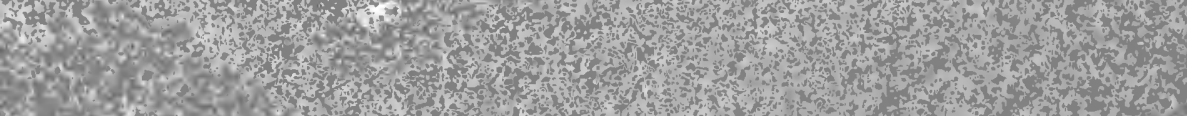

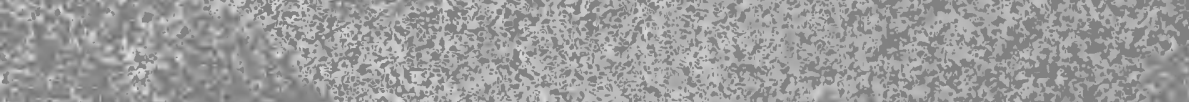

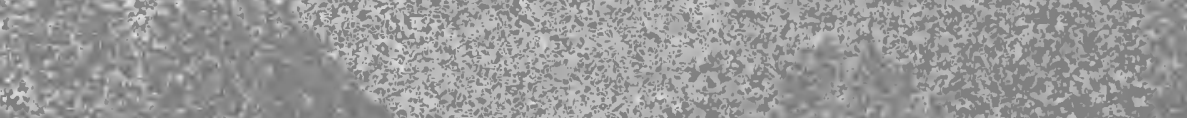

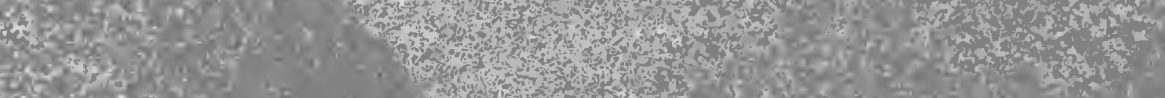


[Reprinted from the Modern Language Review.

Vol. VI. No. 4. October 1911.]

[All Rights reserved.]

\section{AN ANGLO-FRENCH LIFE OF ST OSITH}

THE manuscript from which this life is taken-Welbeck $\mathrm{ICI}$-is sufficiently well known to readers of the Modern Language Review to need no further description ${ }^{1}$. In the fourteenth century it belonged to a nunnery at Campsey, near Woodbridge in Suffolk ${ }^{2}$, where it was used for pious readings at mealtimes ${ }^{3}$. It contains the following thirteen lives: (1) St Elizabeth of Hungary, (2) St Panuce (Paphnucius, probably by Bozon), (3) St Paul the Hermit by Bozon, (4) St Thomas of Canterbury by Garnier de Pont-Sainte-Maxence, (5) St Mary Magdalene, (6) St Edward the Confessor, (7) St Edmund of Canterbury, (8) St Audry of Ely by a certain 'Marie,' (9) St Osith, (10) St Faith by Simon of Walsingham, (11) St Modwenna, (12) St Richard of Chichester by Peter of Peckham, (13) St Catherine of Alexandria by Sister Clemence of Barking 4 .

It will be noticed that no less than seven of the above lives are those of British saints ; many of them exist in no other manuscripts.

The legend of St Osith or rather Osgitha, is a hopeless tangle of anachronisms if we are content to follow the text printed in the Acta Sanctorum, III, Oct. 7, pp. 936-44, nor can the skill of the editor reduce its contradictory statements to order. Much of the conflicting data disappears, however, when we only take into account the simple

1 See Vols. IIr, p. 374, Iv, p. 491 . Romania, xxxII, p. 637.

2 Cf. my article, Romania, xxxvir, p. 418.

3 The MS. bears the remark : 'Ce livere [est] deviseie a la priorie de Kampseie de lire a mengier.'

4 No. 1 has been published by Prof. L. Karl in Zeitschr. fiur vom. Phil., xxxIv, 295 ; Nos. 2, 3 and 12 by the present editor in Romania, xxxvir, 418; Modern Language Review, Iv, and Revue des langues romanes, July 1910 respectively. No. 4 has been published by Hippeau (Paris, 1859) and Bekker (Berlin, 1838) but not on this MS.; Prof. Oelsner has an edition in hand on all the MSS. No. 5 has been published by Reinsch in Archiv f. $d$. Stud. $d$. neu. Sprach., LxIv, 15-94, and Prof. Karl has given the variants of the Welbeck MS. in Zeitschr. fulr rom. Phil., xxxIv, 362; No. 6: see the article of P. Meyer in Romania, xx, p. 43, where 186 lines are printed; No. 13 by Jarnik: Dvě Verse starfrancouzské Legendy, Prague, 1894. I have nearly ready for the press an edition of St Modwenna and in an advanced state editions of Nos. 7 and 10. I have photographs of all the remaining lives and am preparing editions of them. 
recital contained in the oldest of the MSS. extant, viz., Bodley 285. So straightforward does this version appear that $I$ have decided to print it as footnotes to the French text at such places as its narrative fits the French poem. Readers will then see how the legend has grown and be able to decide what may be regarded as interpolations in our text.

The story as contained in MS. Bodley opens with the list of the pious descendants of Penda of Mercia, who, though remaining till the end of his life a heathen, permitted his relatives to embrace Christianity: then comes the betrothal of Osgitha to Siherus, king of the East Saxons, the reference to the various devices by which she prevented her husband from consummating the marriage, the announcement of the appearance of the white stag, the chase, Siher's return after many days to find that Osgitha has profited by his absence to receive the veil from Ecca and Bedewin; Siher's grief at the decision of his wife; his gift of the manor of Chich as a dowry and the precise date 653. The next items are the martyrdom of Osgitha at the hands of pirates, the incident of her carrying her head into the Church of St Peter and St Paul, the smearing of the church door with her bloody hands, her burial at the entrance of the choir. After some miracles have been worked at her tomb, her body is first translated to a place in the middle of the choir and then, under Bishop Maurice of London (1087-1107), the body was again removed and placed behind the high altar. The French poet takes no account of the next item-the glass ampulla and the miracles worked by the balsam it contained ${ }^{1}$, but a reference is made in the text of the Acta $(\S 11)$ and in the MS. Cotton, Tiberius, folio 248 to the healing of a deaf-mute. Finally our MS. mentions that Richard (1108-28), successor to Maurice in the see of London, honoured the saint and settled regular canons at Chich in 1120; it suggests that large numbers of miracles were worked by the virtues of the holy Osgitha but mentions one only which took place in the times of Bishop Richard. This is the story of the theft of the piece of marble by German sailors who called at the mouth of the Colne during their voyage from Yarmouth to Antwerp (11. 857-1064).

It has been objected that the legend of St Osgitha as above related, puts Siher ten years and the bishops Ecca and Bedewin twenty years too early, but Siher is mentioned in Bede $(111,30)$ for the year 665 as returning to heathenish practices during a plague in his dominions and as being re-converted by Bishop Jaruman and there seems no reason why he should not have been married to Osgitha twelve years previously;

1 It should be noted that MS. Lansdown also omits this, see footnote to lines $825-56$. 
again the appellation of Ecca and Bedewin as 'pastores et pontifices' is not conclusive evidence that they were bishops at the time Osgitha took the veil, as the second term may be merely anticipatory-the French text says of them:

Prestres esteient ordinez

Et seinz evesques puis sacrez (417-18).

The first addition to the legend we may take to be posterior to Richard $^{1}$ bishop of London who died in 1198. There is no evidence for it outside the French text which tells that this bishop coveted the wealth of the canons of Chich-their importance may be adduced from the fact that their first prior, William de Corbeuil, became archbishop of Canterbury in 1123; and their wealth from the imposing list of benefactors and manors and that at the time of the suppression the priory was valued at over seven hundred pounds per annum-and sent his seneschal to forbid them to enjoy the emoluments of their office. This is a curious side-light on the character of this bishop; for from the Vita magni sancti Hugonis (Hugh of Lincoln). we learn that in November 1197 there came a demand from King Richard for 300 knights, or money sufficient to hire as many mercenaries, to serve against Philip of France. The Archbishop (Hubert of Canterbury) convened a council of bishops and barons at Oxford, and some of the bishops were evidently indignant at the command for we have the Bishop of Lincoln protesting: ' I know that the Church of Lincoln is bound to provide military service for our Lord the King, but only in this country ; outside England no service is due.' The Bishop of London, on the contrary, speaking as Dean of the province, declared his willingness to comply with the King's demand, wishing evidently to stand well with the King and recoup himself from the possessions of the canons of Chich. For his punishment I know no authority but the French text; in William of Malmesbury, however, we find that Richard-the founder of Chich-suffered from paralysis and intended to retire to Chich; the two Richards must have been confused. The second addition we must consider to be the connection of Osgitha with Modwenna. Leland in the Itinerary, viII, ii, fo. 92, gives the heads of the life of Osith ascribed to a certain Albericus Verus (see above, footnote to Richard) canon of Chich and dated 1250. To him is perhaps due the

1 Richard Fitz-Neal, chancellor and author of the Dialogue on the Exchequer, cf. Schofield, Anglo-Latin Literature in English Literature from the Conquest to Chaucer.

The reference in Dugdale (Stevens' translation, p. 140) is as follows: 'St Osith's Priory at Chich in Essex, founded by Richard Belmeis, bp. of London, with a design to have resigned his bishoprick and become a Canon Regnlar himself but that he was prevented by death. Albericus Verus figures here as one of the benefactors.' 
connection of Osgitha with Modwenna. One must remember that there are probably three different holy women of the name of Modwenna (or Monenna) whose deeds go to build up the story of that saint. The connecting link between Modwenna and the subject of our manuscript must be looked for in the similarity between the list of the pious ancestresses of Osgitha and the various Mercian princesses who lived in religion and were connected with Modwenna; the Edith (daughter of Ethelwulf) of Streaneshalh (Whitby) must have suggested the Edith (daughter of Egbert) of Streveshal in the forest of Arderne-Warwickshire and companion of Modwenna-the names are constantly confused in the MSS. ${ }^{1}$ In the lives of Modwenna, both in that of Conchubranus and in the later one of Geoffrey of Burton, appears a maiden Osid or Osith but no mention is made in either of her forbears. It is not clear from which of these the writer of the Latin text published in the Acta took his story of the three-day immersion of Osith in the river Anchora but the French poet probably knew the version of Conchubranus, and not that of Geoffrey as Hardy (Descriptive Catalogue, I, 2, No. 1097) suggests. In Conchubranus alone (II, 8) we find a mention of Osith accompanying Modwenna to Rome (cf. line 211) and in it the bridge is said to be 'unum lignum' (II, 9) like the French 'd'un sul tref' (line 275). It will be noted that the adapter of the Bodley text says he will tell only one miracle-that of the German sailors-he may probably have known, though he omits, that of the paralytic woman which is told in an abbreviated form in the Acta and the Cotton MS. ${ }^{2}$ Her lapse from the paths of righteousness, punishment and second healing are similar to the previous story in the same text, where a girl whose arms are paralysed had been cured, but breaking her vow of continence by marriage, the same penalty is imposed and on further repentance she is again healed (AA. SS. §11). As to the other MSS. mentioned by Hardy, MS. Tanner 15, pp. 445-8 is identical with the text of the Acta, MS. Lansdown 436 ff. 29, 30 is identical with Bodley 285 except for an occasional change in spelling or word order and a number of omissions

1 Cf. the excellent edition by Esposito in Proceedings of Royal Irish Academy, Vol. xxvII, and the MSS. Mostyn and Royal, $15 \mathrm{~B}$. Iv, which will be printed in my edition of Modwenua.

AA. SS. \$ 11. 'Mulierem contractam Virgo sancta erexit.

- Puella quædan officio brachii adeo privata, ut lateri ejus inseparabiliter inhæreret, cum meritis S. Osithæ perfectæ sanata esset, votun continentiø nuncupavit; sed postea cum quodam juvene matrimonium contraxit. Ei S. Ositha apparens, cur temerario ausu vota, quæ distinxerunt labia sua, rupisset, spurcissimum conjuginm improperando, redarguit, et compedum vinculo invisibili pedes ejus arctius constrinzit, ut pedem pedi subjectum divellere non.valens officio pedum prorsus destituta ultionis divinæ flagellum sentiret. At tandem tamen meritis sanctæ virginis sospitate reddita, correctius deinde vivere studuit.' 
which we note by $(\quad$ ) in the text here printed, while Bodley 240 is a series of Lectiones'.

Our author (or authors, cf. below) does not appear to have used any of the MSS. that have been enumerated; thus he mentions Osgitha's birthplace as Querendon (line 184) as does Leland (quoted by Dugdale); in the chase King Siher pursues the stag as far as Dunwich (line 616) which seems to be an addition of his own; the names of the pirates responsible for Osgitha's execution are Ynguar and Ubba (line 770) known only from the Anglo-Saxon Chronicle for the year 870, and they are mentioned as the slayers of Eadmund both in the poem (line 776) and in the same year in the Chronicle. According to the Chronicle these Danes came down from Mercia and settled at Thetford and pushed their plunderings thence, while in the French poem they landed near Chich (line 792). Then, too, as has been pointed out, the whole story of Richard's persecution of the canons of Chich and his punishment rests on no known source. That the sources for a poem should only be preserved in outline is unfortunately very common. It happens only rarely that a French poem and its Latin source form a close parallel as is the case with Peter of Peckham's life of St Richard of Chichester and with St Modwenna; this is natural, however, when we consider that the mass of material was so great that 'abbreviationes' were necessary and that these, put into the order of the liturgical year, form the legendaries which are in so many cases only preserved ${ }^{2}$.

The portion of the Welbeck MS. which contains the life of St Osith is in a good late thirteenth century hand and very legible; the MS. we may suppose to have been put together early in the fourteenth century - there are three different hands and perhaps more ${ }^{3}$; we may assume that our portion dates somewhere about 1260-70. On reading the poem we are struck by a discrepancy in the language and scansion. The early parts dealing with Osith's youth, i.e., the first 180 lines or so-the marriage, the taking of the veil; the chase; the martyrdom; the incident of the sailors; the paralytic woman-as we shall state in greater detail later, do not appear attributable to a date later than the end of the twelfth century. The last incident concerning Bishop Richard must be later than his death in 11.98 but probably very little later; while the Modwenna story may belong to the second half of the

2 I have to thank Mr Winstedt of the Bodleian Library for this information.

2 See Paul Meyer's articles in Histoire litteraire de la France, xxxIII, Notices et Extraits, xxxvi, etc., etc., also the English Legendary in E.E.T.8., Vol. 87.

3 Prof. Karl has promised a fuller description of the Ms. in a forthcoming number of the Revue des langues romanes. 
thirteenth century. In the paragraphs dealing with the language and versification we call this episode (B) and the former (A).

As has been often stated, Anglo-French is not a distinct dialect showing normal and regular development, and phenomena that would be adequate for approximate dating in other dialects are useless here unless supported by other evidence. It may be well however to enumerate certain linguistic traits and to compare them with other AngloFrench works; for this purpose we take the Vie de saint Gilles of Guillaume de Berneville, the works of Simund de Freine and those of Chardry, dated by their editors at about 1170, the end of the twelfth and the beginning of the thirteenth century respectively.

1. Cases of the confusion of $e<$ Latin $a$ with $i e<a$ preceded by palatal or $\breve{e}$ occur with ever increasing frequency till by the beginning of the thirteenth century rimes where the two sounds are kept distinct are due only to chance. Our text only offers cases of this confusion in the parts called (A) and (B), cf. $\S 17$. This must be due to some special reason, which I have been unable to determine and which I think could only be determined if we had sufficient manuscript material for a sound critical text. Even if we could consider the whole poem, except the episode (B), now published as of one writer and one period, the fact that only three instances of this confusion occurred would still be remarkable. This phenomenon cannot help then for the dating of the poem; it might point to the anthor being a Frenchman of France if other facts bore this out, but they do not seem to do so.

2. From the foregoing we shall understand that there can be no rimes such as $c e l<$ colum : $e l<$ alum for aliud; this is also true for St Gilles (which, however, confuses $i e ́$ with $e$ ) whereas Simund and Chardry know this confusion.

3. Confusion of infinitives in -er <-are and those in -er (for eir) $<$ ére is unknown to St Gilles and our poem, whereas cases of this are in Simund and Chardry; cf. Rom. Forschungen, I, p. 210.

4. Confusion of infinitives in -ier with those in $-e r$ (for eir) < ére does not occur in St Gilles and in our poem only in (B), cf. § 17, but there are certain cases in Simund and in Chardry.

5. -ai and -ei final; these vowels are kept distinct in St Gilles and in our poem, but in Simund and Chardry there are cases of confusion. This confusion seems to date from the last decade of the twelfth century.

6. -eie and -ée final. This rime only occurs with the word veie < via and a few past participles of the first conjugation, cf. 1l. 811, 1185, 1207, 
1333. This is fully discussed in $\$ 15$; although the spelling -ei for $-e$ occurs in many texts $I$ have not met it in rime with -é except in Frère Angier (1212-14) and Chardry, who has monée < moneta in rime with dunée and aportée [Sept Dormans, 1205, 1291].

7. -ais and -eis. Our poem has no instances of rimes in -ais and only one in -eis, viz. reis : Engleis 131, cf. $§ 15$, nor does it mix -ais with -es; it has pure rimes in open $e, \mathrm{cf} . \S 3$. This must be due to chance as cases of this confusion are found in Gaimar, Adgar as well as in St Gilles and Simund. In spite of Koch's assertion that Chardry keeps the sounds separate, we have curteise : pleise and treis : mauveis in the Petit Plet.

8. -ait and -eit. These sounds are kept distinct in St Gilles and in our poem, cf. $\$ \S 13,15$; some hesitation in Simund and Chardry.

9. -air and -eir; e.g. detraire : creire. These are distinct in St Gilles ; our poem contains one case viz., feire : trere 1195, cf. §15. Confusion is frequent in Simund and Chardry.

10. -aire and -ère; e.g. faire : terre; confusion in St Gilles and in Simund and Chardry; our poem, probably only by chance, has these rimes distinct, ef. $\S 3$.

11. $-u i$ and $u$. This change of $-u i$ to $u$ does not take place exclusively on English ground; before $s$ it is common in Norman. In St Gilles the sounds are kept distinct; in our poem confusion is only found before $s$ and $t$. In Simund the change occurs in addition before $r$, e.g., dedure : dure [S. G. 123], while in Chardry it is found even when the vowel is final, e.g., ennu : vertu [Jos., 2925].

12. -eus <-alis or -illos; these endings are distinct in St Gilles but confused in Simund and in Chardry; there are no instances in rime in our poem.

13. - an and -en + consonant. No confusion in St Gilles, our poem nor in Simund, whereas Chardry has some cases of confusion, viz., obeisanz : cumandemenz [Jos., 555].

14. -ain and -ein. These sounds are not found riming together in St Gilles, but our poem has main : sein < sinus 667 . In- Simund and Chardry confusion is common, cf. $\S 14$.

15. -ien. The rimes on this vowel are pure in St Gilles, in our poem and in Simund. There is of course the regular variation with nient, ef. $§ 18$.

16. $s$ and $z$. These consonants are kept distinct in rime in St Gilles, in our poem and in Chardry; there is one case of confusion in Simund, cf. $§ 32$. 
17. -eit added to verbs of the first conjugation unknown to St Gilles and to Simund is found in our poem only in (A) and (B). In Chardry this mistake is regular, cf. $\S 55$, iv.

18. The feminine $e$ is rarely mute in the body of the poem, very frequently in (B) and less frequently in (A), cf. $§ 5$.

From this rapid survey it appears that the bulk of our poem must be placed near the end of the twelfth century and that it is probably older than the works of Simund de Freine and of Chardry. The other portions may, as I have already suggested, be placed in the early part and in the second half of the thirteenth century respectively. The reasons for the divisions (A) and (B) will be made plainer in the paragraphs dealing with versification.

Ici comence la vie seinte Osith virge e martire.

(5eo nus mustre seinte escripture $\checkmark$ Bon fu ki met en Deu sa cure, Et aime e creient son creatur

4 Plus ke ne fet autre seigniur; Ki l'aime e creient e bien le sert, Ne ci ne ailliurs ja ne pert; Ki guerpist terre pur son non

8 Ciel li donne de gueredon; Ne change cil pas follement Ke terre lesse e le ciel prend; Ne follement ne change mie

$\mathrm{v}^{\mathrm{S}} \mathrm{b}$ Ke lesse mort e receit vie; Cil change bien, eil change a dreit $\mathrm{Ki}$ mort lesse e vie receit; Kar certes del mund la richesse

16 N'est fors dolur e granz tristesce; Honur del mund est trespassable, Et a nus tuz mut poi estable. $\mathrm{Ki}$ ne volt creire ne saveir,

20 Bien l'os dire, fols est pur veir. Veum par ceus ke sunt aléNos ancestres e trespassé; $U$ sont nos aels e nos peres

$24 \mathrm{U}$ [sont] nos uncles e nos meres, $\mathrm{Ke}$ tant furent riches e beaus, Assez orent dras e chiviaus?
Tuz sont alez, sachez en fin, $28 \mathrm{Si}$ vous atendunt en chemin;

Quel jur son cire ert aprestez; Pur ceo vus di, si l'entendez, $32 \mathrm{Ki}$ aime Deu bour fu nez. Kar tant cum il Deu amera, $E$ son servise meintendra, Pur veir vous di, n'estut doter 36 Quel jur deive del siecle aler. A Deu tant bonz fu finement Ke de seinz Deu example prent, $\mathrm{Ki}$ guerpirent terre e honur

40 Et tut le mund pur Deu amur, Et soffrirent hunte e esclandre, Pur Del firent lur sanc espandre; En hone entente e (en) bon espeir 44 Mort donerent pur vie aveir; De tels a grant plente trovum

195 En seinz escriz ke nus lisum; Et nient de madles solement,

48 Mes de femmes tuit ensement. Seintes (e) veraies Deu anceles, Et d'alquantes tendres puceles, Ke tut le mund unt refusé

52 Pur prendre sei a Dampnedé(u), Et suffrirent pur son non Ou veraie conpunction. De une tele volum parler

\section{Cf. § 44 iii. \\ 6 Cf. $\S$ on Hiatus. \\ 21 MS. čeus par ke...}

32 MS. bonz: the emendation seems certain, for the expression bor (beor, buer, etc.) est nez is excoedingly common, of. line 1424, but it does not render the line correct in scansion. Cf. \$ 64. It is interesting to note that the bor $i$ alasses of Alexis, 90 e figures in one MS. also as bon....

37 The reading bonz should perhaps be again emended, the sense would be improved, though the tense is awkward.

$38 \mathrm{ke}=q u i$, cf. $\$ 49$.

48 ui for a (0), cf. $\$ 10$.

50 MS. de qüz.

53 The line might be corrected by inserting mut before suffirent; the verb requires an object, cf. vv. 41, 42 above. 
$56 \mathrm{Ke}$ durement fet a loer,

Ke Deu ama e Deu servi,

Et tut le mund pur li guerpi ;

Et pur son verai creatur

60 Guerpi son terrien seigniur.

Li reis estoit fort e puissant

Pur Deu l'ad tut refusant,

Pur li suffri peine e dolur,

64 Et martyre a chef de tur.

La virge dunt [vus] voil parler

Sovent avez oy nomer,

$\mathrm{Sa}$ vie n'est guerres leue,

$68 \mathrm{Ne}$ cum dreit fust par tut seue ;

Ele est par non Osyd nomée,

De Engleterre nurrie e née;

A Deu voua son pucelage,

$72 \mathrm{E}$ bien le tint tut son [e]age ;

$\mathrm{Sa}$ vie est bele e gloriuse,

Seinte e duce e preciuse ;

En cest romanz purrum oir

$76 \mathrm{~A}$ prendre bien e retenir De seint Osith e de sa vie, Cum Deu la choisi a amie;

${ }_{r 0 \mathrm{~b}}^{185}$ Cum Deu l'aveit amé et chere-

80 Mustré l'ad en meinte manere;

Par miracles e par vertu

Ke en plusiurs lius sunt avenu,

Et bien veu par Engleterre,

84 Et en la peis e en la guerre;

Ke Deus i ad fet apareir

Et nuit e jur, (e) matin e seir ;

Entendez i communaument,

$88 \mathrm{Kar}$ ge le vus di seurement,

Meuz vaut oir ici entour

Ke de la geste paenur,

De Guercedin e de Saisons,

92 Deu enemis e felons,

E d'autre teus pur verité,

U l'em vus ment a grant plenté ;

$\mathrm{Ky}$ aime e ot la vanité,

96 Dou li en set mut [de] malgré.
Des seinz Deu la veraie estoire Devum aver bien en memoire; Kar quant nus la folur oum,

100 Essample sovent en pernum; Ky sen escute e sen entent Il en amende mut sovent.

De seinte Osith or(e) vous dirum,

$104 \mathrm{Si}$ cum en l'escrit le trovum;

Certes mut fet a amer,

Et a criendre e a duter;

Mut par est bien de Deu lasus,

108 Bien le direz kant orez plus;

Mut est dutuse a curucer,

Kar tantost pense sei venger;

N'i ad mester coruz ne plait,

${ }_{v^{\circ} \text { a }}^{185}$ Tost se venge ki se mesfait,

Cum vus avant orrez assez;

En cest romanz si l'entendez,

Ne serrez de fables peu,

116 Mes de miracles e (de)vertu, Ke Deus en Engleterre fist Pur seinte Osith k'a li se prist; $\mathrm{Si}$ en crei ke volenters l'orast

$120 \mathrm{Ke}$ seinte Osith gre le sav(er)ast; Kar ki voudra bien peut aider Vers Dampnedeu e avancier. Ore entendez bien dunc sa vie

$124 \mathrm{Ke}$ en puissez aver aie, Sucurs e son amendement Vers Dampnedeu omnipotent. La virge Deu tant bonurée

$128 \mathrm{Ke}$ seinte Osith est apellée, Gentil esteit de parenté, Fillie ert au rei mut renomé, En Engleterre esteit cil reis,

132 Fredeyold l'apelent Engleis. Seinte Osith ot cil rei a pere, Withborc reine fu sa mere

Ke esteit fillie Pende le rey,

136 De grant puissance e de nobley, Et mut out en subjection;

64 Cf. \& on Hiatus.

62 Cf. \& 56 corr. li va refusant.

65 The spelling virgine (cf. 1. 382) could be adopted instead of amending vus.

74 Cf. \& on Hiatus.

89 It is common in Agn. didactic poems to find epic and lyric poetry decried; cf. my note in Vie de Saint Richard, l. $c$.

91 The king of the Saxons in the Chanson des Saisnes of Jeau Bodel; a large number of variants of the name exist, but this seems to be a new one.

92 Some word seems to be missing from the text, perhaps mals.

97 Cf. $\$ 20$.

105 A small space is left in the MS.; perhaps ele should have been inserted. Reasons why St Osith should be feared are furmished by the miracles.
106 Cf. $\S$ on Hiatus.
119 Cf. $\$ 53$.
120 Cf. $\$ 59$.
121-2 Cf. $\$ 55$.

vv. 126-50 Beata virgo et martir Osgitha ex nobilissima Anglorum stirpe extitit orta. Erat filia Fredeswaldi regis (atque Wilburge regine, filio Pende regis de quo Beda in tercio ecclesiastice Anglorum istorie libro mencionem facit). Qui Penda licet in paganissimo usque ad finem sue vite permansisset flium tamen christianissimum nomine Peadam et filias neptesque et proneptes sanctissimas habuit, (quarum corpora per Angliam diversis in locis quiescunt). [Bodley, $285 \mathrm{fo}^{\circ}, 121 \mathrm{r}^{\circ} \mathrm{b}$.] Omissions in MS. Lansdown shown by ( ). 
Seint Bede en fait grant mention

En cele estoire des Engleis,

$140 \mathrm{E}$ dist tut fust cil paens reis;

Il out enfanz de bone fey,

Verais en crestiene ley;

Un fiz aveit mut renomé

144 Fillies e neces a (grant) plenté

$\underset{\mathbf{v}^{0} \mathrm{~b}}{185} \mathrm{Ki}$ se pristrent de Deu de tut, Amerent e servirent mut.

Le reis Pende dunt ay parlé

148 Les chers enfanz out engendré

Ke Dampnedeu a sei choisi;

Lur non avez assez oy:

L'une est nomé[e] Keneburc,

152 Et l'autre ad non Eadurc, Seinte Osith fu del parenté, (Kar) lur niece fu pur verité; Example prist de lur chasteté,

156 Pur Dampnedeu servir a gré.

Entains ell sa primele enfance

En fiz Deu aveit sa fiance;

Entente e tute s'amur

160 Aveit on Deu son creatur;

Richesce aveit a grant noblei

Si cume fillie a riche rei;

Plente de vivre e de manger,

164 Et quanque l'em en mund ad cher;

Mes trestut ce petit proisa,

Pur Deu despit e tut lessa,

La richesce ne tut le bien,

$168 \mathrm{~K}$ 'en mund veeit ne preise rien;

Bien le sachez ke la pucele

De face esteit e clere e bele,

De cors bien fete c accmée,

172 Mes plus dedenz fu csmerée;

Riches e povres refusa,

Son pucelage a Deu voua,

Et cil requist come scigniur

$176 \mathrm{Ke}$ violée ne seit nul jur;

Et Dampnedeu bien l'en oy,

${ }_{\mathrm{r}_{\mathrm{a}}}^{136}$ Par sa ducur e sa merci

Ne perdi sa virginité(e),

180 Pur nul home de mere né(e);

Unc (nul) hom de ce ne la conut, Virge nasqui, virge morut.

La vile $u$ seinte Osith fu née

184 Querendone est apellée;
Assez sevent li païsant

K'en la contrée sunt manant, Plusurs [del] luitain autresi,

188 Le liu $u$ seinte Osith nasqui; Enseignie i ad assez apert, Ke puet chescum fere cert, Unkes puis jekes a cest jur

$192 \mathrm{Au}$ lin n'out herbe ne verdur : Par son nestre est le liu sacré, (Et) de tut humein us sequestré; Dementers k'en ceste vie fu,

196 Deu fist pur lui mut grant vertu, Et pus k'est martir mut a fest, Dunt partie orrez si vus plest, Pur vus joir ge [vus] dirray,

200 Une partie ke apris ay;

Si cum avant [ge] vus disai[e]

Kant des parenz Osith tuchai[e];

Seinte Osith out grant parenté

$204 \mathrm{De}$ grant richesce e (de grant) seinteté,

Aels e uncles de graut poesté, Auntes e neces de grant chasteté; $\mathrm{Li}$ plus[or] d'eus sunt assemblé,

$208 \mathrm{Au}$ pere Osith sunt conseillié Ke a tel mestre seit Osith baillié[e] $\mathrm{K}^{\prime}$ en nule manere seit afolé[e].

${ }_{r \circ}^{196}$ A Modwin baillier a plus plout,

212 A cest consent le conseil tinout, Kant la pucele parler saveit, A Modwen l'abesse haillie esteit;

A merveille ama e chere tint,

216 A Rome fu, ou li revint. Ceste Modwene dunt ay dist, Juste Ardene deus musters fist; L'un a Polesvurche ce dient la gent,

220 L'autre en Stranesrale vereiment. Modwen en l'un sujurna, A Edith l'autre otreia;

Al rei Edfrid esteit seur,

224 Modwen ver li out grant amur.

Un jur issi aveneit

K'en oreisuns Modwen loinz aleit; A Edith Osith enveia

$228 \mathrm{Ke}$ bien la preist kar mut l'ama; Sa compaigne out long tens estée Mut fu de lui joiuse e lée;

155 See $\$ 70$.

157 Cf. $§ 59$.

vv. 151-6 (Nam sancta Keneburga et sancta Edburga ejus filie sancta vero Mildridtha neptis et sancta Werburga sanctajue Elstreda et sancta Elgida proneptes ejus fuisse testantur cronica.) [Bodley, ibid.] 172 Cf. $\$ 65$.

$184 \mathrm{Cf}$. on Versification. For iks see Introduction. Quarrendon is a village $1 \frac{1}{2}$ miles N.W. of Aylesbury. There exist the remains of an early church.

185 Cf. \$ 31, and English tyrant, parchment and their French equivalents. Cf. 1. 589 and $\$ 31$.

190 Cf. $\$ \S 59,65$.

211 For the Modwenna episode, cf. Introduction.

218 MS. teus.

225

Cf. $\$ 65$.

221 Cf. $\$ 65$.
205 Cf. 870.

212 Cf. \& 68.

223 Corr. serur, cf. $\$ 43$, iv. 
Aukes [de] tens ert trespassé,

232 Modwene a muster est repairé, Un livre out trové Edithz, Plein de proverbes e (de) bonz diz; Examples i trova a grant plenté,

236 De vertuz e de seinteté;

$\mathrm{Ne}$ vot cel bien sule celer,

Mes ou Modwen comunier,

A volage ne vot baillier

240 Ki l'empeireit de leger.

Osith apella par grant ducur:

'Plereit, dist ele, bele seur,

'A nostre mere Modwen aler,

${ }^{186}$ 'A li cest. livre par moi porter;

245 'Dirrez ke granz biens i puet trover, 'Dunt se memes puet amender,

'Et par doctrines k'ele trovera

248 'Tuz les sens amender purra.'

Osith encline, lui otria,

Dist ke volenters i irra;

Le livre prent, rien ne resta,

252 Mes al aler se [a]presta.

Ha Deu ke Edith ne seust l'aventure

K'avendreit a Osith en ceste ure,

Ne la lerreit aler (si cum) je crei

256 Pur tut l'or Mide le rei.

Si Edith seust ke li avendreit,

Cest(e) eire emprendre ne li suffreit

Pur tut l'or ke seit en Espaine,

$260 \mathrm{Ne}$ pur [tut] l'onur de Lovaine.

Si Edith seust ke Osith est a venir,

Cest message ne li freit furnir,

Pur ce k'ot Salamon le sage

$264 U$ Alisandre en tut son age.

Mes Deu li cela cest conseil,

Kar de Osith vot fere grant merveil.

Ivern ert e mut out pleu,

268 Pur ce la pucele n'est (re)mansu; A l'aube del jur (en) la matinée,

Errant se est acheminée;

Le livre prist e s'en ala

272 A Modwen u Edith l'enveia.

En cest chemin une ewe curreit,

$\mathrm{U}$ la damisele passer deveit;

Le punt fu lung e d'un sul tref,

'276 Passer comensa pouruse e suef

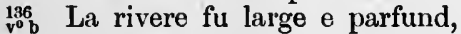

Et ele ert ja en mi le punt;

Le vent fu fort e mut bruant,

280 Les pans de son mantel despant, Mes par les taches al col remist, Et par les gerrons a sei (le) prist
Son mantel ke le livre obli,

$284 \mathrm{Ke}$ de ses meins en l'ewe chai;

De cele perte fu esbai,

Al prendre s'abessa si le suivi ;

(Bien) cuida son livre aver receu,

288 Mes amdeuz l'ewe ad reteneu.

Loinz de cel liu les desaka,

(Et) en une krenke les jeta,

[Et] bien treis arpenz loin del pont,

292 Les graventa en un parfunt,

La jurent amdeuz treis jurs

Et treis nuiz sanz [aucun] succurs;

La jurent treis jurs e treis nuiz

$296 \mathrm{Si}$ enfundre en un puiz;

Treis jurs e nuiz Osith i jut

Iloec neé en cel duit.

Issi Osith gesir larrum,

300 Et de sa dame nus cunterum.

Del quart jur s'esclarzi la matinée,

Edith s'est mut esmerveilliée

Ke feist Osith tant targer ;

304 Dist k'ele irra l'encheson saver.

En son quer suffri grant batestal,

Bien leva devant le chant de gal,

Errant se mist a cheminal;

308 Modwen l'encuntre par esperital;

Si cum Edith aveit veu

${ }_{r^{0} \mathrm{a}}^{187}$ Modwen silence ad derumpu;

Bien par son semblant ad aparcu

312 K'ele fu dolente et irascu.

'Edith, bele seur, k'estes dolente?'

'Dame: (dit Edith) ore oez qui me turmente,

'Damoisele Osith vus enveiay,

316 'Un livre portant ke mut amay,

'Hui est le quart jur trespassant

'Puis n'en oi mes ne cuntrement;

'Dame, k'est ele demoré tant?'

320 Dist Modwen: de ço' 'ne soi rien avant.'

Si l'une e l'autre fu esbaye,

Ceo demander serreit folie,

Pasturs furent leus delez,

324 Lur bestes pessanz par ces pres;

Les dames a eus sunt alez

Apres saluz unt demandez:

'Amis ke Deu vus doint sauveté,

328 'Ceo dunt vus prium diez verité,

'Veistes une damoisele al punt aler,

-A la matinée li tierz jur fu er,

'Afublé out un neir mantel?'

332 A ceo dient li pasturel :
239 volage, cf. $\$ 59$.

$253 \S 53$ vii.

283 MS. ken.

296 Cf. $\$ 59$.
245 Omit ke, cf. $\$ 53$ I. iii.

281 Cf. \$ 59.

293 Cf. \$ 65 .
261-2 Cf. $\$ 59$.

290 krenke, cf. \$ 59.

306 Cf. $\$ 48 a$

appears to be very rare. 
'Une tele pucele cum vus querez,

'Hui est quart jur veimes assez,

'Amunt sur la chause passer,

336 'Jeske al punt veimes aler, - Mes kar a nos bestes entendium 'Plus n'en seumes en fei vus dium.' Lors ke neé[e] fust suscherent

340 Les dames e al punt repeirerent; Amedeuz se mistrent en oreison, Ou plurs ou grant devotion, ${ }_{r^{\circ} \mathrm{b}}^{197} \mathrm{Ke}$ Deu lur deignast le cors mustrer,

$344 \mathrm{Ke}$ suveus le puissent enterrer, Primes sa preere Modwen fina, A pont Osith deuz feiz clama: 'Damoisele Osith venez hors

348 ' Ke veer puissum vostre cors; 'A ceo vus doint force e vigur ' Ke Lazre suscita al tierz jur.' A peine ont Modwen sa voiz fini

$352 \mathrm{Ke}$ de l'ewe Osith ne issi, Neste e secke son livre ausi, Et dist: 'dame veez moj ei.' Si cum del tut fu virgine pure,

356 Son livre e li sunt sanz muilliure. $\mathrm{Ha}$ Deu ky [dune] pust esgárder, Quel joie les dames vunt demener. Tut le mund dust [il] refuser,

$360 \mathrm{Ki}$ ces treis pust bien aviser. Modwen Osith ad a sei prise, Tant cum vesqui li est remise, Tant cum Modwen fu en vie,

364 De li Osith ne fu partie. Ke ja ne seit eelée cest miracle, Cum Osith en l'ewe out habitacle; L'eune ou Osith fu neé[e]

368 Tut dis puis en ad renomée; $\mathrm{Ke}$ ja mes en secle ne seit celée, Le liu Ninnepol est apellée; Ke dune fust numein ne di pas,

$372 \mathrm{Kar}$ l'en le tendreit a folie e gas Si dune fust nonein velée Ne fust apres reine espusée. Assez a[i] dist de cest veage, ${ }_{v^{\circ}}^{197} \operatorname{Or}(e)$ vus dirrai del mariage;

377 Kant Modwen fu en cel mené,
Frethuuald sa fillie ad repellé.

Le sunt e joius (tuit) li parent

380 De sa porture e enseinement;

Tuit fu son purpos e desir Virgine vivre e [virgine] morir. (En quel ehange serra martir) De cest purpos n'est ensensé

384 Son pere, pur ceo s'est purpensé D'Osith, k'il la volt marier' A poestif homme le rey Syer. Seinte Osith en est anguisuse,

388 Pensive en quer e doleruse.

Mes al pere de ceo n'est rien, Que que li seit eu mal eu bien, N'i ad rien d'escusation,

392 Faire l'estuet eu voillie eu nun.

Li rei fet mander ses amis,

Baruns e cuntes del pais, $\mathrm{E}$ ehivalers o autre gent,

396 Pur lur conseil communalment. Al rei Syer ad fet douer Osith sa filie e espuser, Solune la gard ke fu asis

400 En icel tens en lur pais. Syer le rey sa femme ameine, Ele out au quer dolur e peine, E Deu requert omnipotent

$404 \mathrm{Ou}$ lermes mout espessement, $\mathrm{Ke}$ ja ne li seit violé Ceo que peça li out voé, Sun pucelage li purgart

$\underset{v^{\circ} b}{187}$ Ke ne perde ne tost ne tart; Mes si cum s'est a lui donée, Bien li puisse estre gardée. Le jur ke seinte Osith alat.

412 Kant li rey Syer l'enmenat, Witburg sa mere al departir Deuz homes fist ou li venir, Ke Dampnedeu aveient cher,

416 Ententif mut a sun mester; Prestres esteient ordinez, Et seinz evesques puis sacrez; Bedewin out li un a nun,

420 Ecca li autre cumpaignun. La reine ad a ceus liverée,

339 Cf. $\$ 59$, cf. Godefroy vii under suschier=penser, most of the examples are taken from Anglo-Norman sources.

341 Cf. $\$ 64$. $\quad 343,344 K e$ may be omitted. Cf. $\$ 53$.

351 Cf. $\$ 59 . \quad 367$ ou, MS. eu, ef. $11.390,392$.

350 Cf. $\$ 66$.

377-8 Cf. $\$ 44$ B. iii.

(n) sense of l'en récompenser. The reading marier seems to make better sense.
386 Cf. $\$ 60$.
390-2 Cf. 824.

vv. 384 seq. (Talibus igitur beata virgo et martir Osgitha orta parentibus) oum [MS. Lans. reads: cum igitur beata Osgitha..] jam adulte esset etatis invita nt postea patuit tradita est in conjugium Sihero regi prientalium Anglorum. [Bodley, ibid.]

vv. 401-84 Qui cum post celebratas nupeias ejus vellet uti connubio et illa ejus lasciviam diversis occasionibus diu impedisset, tandem universis pene consumptis cum quadam die ejus virginitati graviter insiatens illam vi opprimere conaretur. [Bodley, ibid.]

417 Corr. proveire...ordiné seint evesque...sacré. Cf. $\$ 43$ iv and 44 ii. 
Osith sa fillie e comandée,

Pur li garder en dreite fey,

424 En dreit amur e en sa ley.

Li reis Syer ses noces tint

Si cum a rei plus bel cuvint;

E les plus bauz de sun regné

428 Le jur $\mathrm{i}$ avei(en)t ensemblé, Mut se peinat de tuz heiter.

La nuit quant vint puis a cocher,

Sa femme ad fet tost demander

$432 \mathrm{Ke}$ tant al quer puet desirer.

Kant seinte Osith out la novele

A Dieu ceo dist: "la vostre ancele

'Pur vostre nun or defendez,

436 'Le vostre poer i mestez,

' $\mathrm{Si}$ i mestrai trestut le mien

'Ke ne seie pur nule rien

'Hunye a nuit ne violée;

440 'E quant me sui a vous donée,

${ }_{\mathbf{r}^{\circ}}^{138}$ 'Defendez moy cum vostre amye

' $\mathrm{Ke}$ ne seie a nuit honye.'

A tant si est avant venue,

$444 \mathrm{Si}$ tost cum li rois l'ad veue

Mut la coveite mut la desire

Et seinte Osith li dist, 'beau sire,

'Pur Deu merci, kar m'entendez,

448 '(Et) aukes de respit me donez;

'De ceste assemblé[e] entre nus

'Dunt vus estes tant desirus,

'Respit vus requer sire rey,

452 'Si ja voilliez joir de mey.'

Cil la ne vout pur rien oir

Mes tut dis tire a son desir.

Cele pur rien ke sace dire,

456 Par boneirté plus ke pur ire, $\mathrm{Ne}$ vout faire ne consentir

A son talent n'a son pleisir.

Mut requert termes e respiz

460 Mes il le fait mut a enviz;

Et nequedent tant l'ad luctée

Requis[e] mut e travailliée;

Par la vertu del seint esperit

$464 \mathrm{Ke}$ l'oure est mis en respit;

Deus l'ad gardé omnipotent,

Onc ne l'aprocha charnel(e)ment,

$E$ gueres plus ne demora

$468 \mathrm{~K}$ 'il autre feiz la resona

Ke a li se vout assembler,
Respit ne [li] vout plus doner.

Mes seinte Osith cria merci

472 A Dampnedeu tut autresi Cum aveit ele fet devant

${ }_{\mathbf{r}^{\circ} \mathrm{b}}^{198} \mathrm{Ke}$ par son nun l'ert defendant. $\mathrm{Li}$ reis comence a losenger,

$476 \mathrm{E}$ tel oure est, a coroucer; Mes tant a purchasé e quis $\mathrm{E}$ tant feit entre giu e ris, De jur en jur est purloinié

480 Charuelement ne ad aproché. Soventefeiz l'ad envaie, Mes Dampnedeu garda s'amie, K'ou son barun maneit issi

484 Virgine treis anz e demi. Un jur apres issi avint $\mathrm{Li}$ reis Syer sa feste tint K'il fist de sa nativité;

488 Grant poeple i out assemblé Contes, barons e chivalers, Ses frankeleins e ses terrers. Le jur quant il aveit mangé,

492 Et sunt partut joius e lé, Et il meimes bien enbeverez En la chambre est tut dreit alez, Et s'i cuche [de]sur sun lit;

496 Kant reposé s'est un petit Sa femme fet a ly venir, Si la comence en lit gisir, Bien entrussement li dist,

500 Mes ne li durra [il] respit. Kant seinte Osith icest entent, Plure des oilz mut tendrement Et vers le ciel la sus garda,

504 Et Dampnedeu mut reclama, Ke par la sue grant vertu, G[a]rant li seit en bon escu.

${ }_{v^{\circ}}^{138} \mathrm{Li}$ reis se peine mut forment

508 De son bon fere e sun talent, $E$ ele a trestut sun poer Se tint cuntre l'ardent voler, Mes come de plus se defent

512 Cil se deve e plus esprent, De son bon fere met pur veir Tute sa force e son poeir, $\mathrm{E}$ jure asez e dist le bien

516 Son cuntredit ne li vaut rien. En ceste anguisse $u$ ele esteit

445 Considering the great regularity of the lines of this episode, we may be justified in supposing that the repetition of mut is due to the scribe; the scansion would be rendered correct by reading $e$ for mut.

459-60 Cf. $\$ 32$

461 MS. luciée.

490 frankeleins, terrers, cf. $\$ 59$.

460 MS. envis.

464 Cf. $\$ 59$.

499 The full form, according to Körting s.v. extrorsus, is estrosséement, which would correct the line, but probably the line should be emended li a dit or dist, cf. $\$ 32$, and then the present form would stand, cf. \$ 52 .

508-13 Cf. \$ 59 . 
E la dolur k'al quer aveit

Seinte Osith ententivement

520 Reclama Deu omnipotent,

E dist: 'Sire Deu, vostre aye,

'Ceste ancele, n'obliez mye,

'Tantes feiz m'avez sucuru,

524 'E mun cors, sire, defendu;

'A ceste feiz, (sire) me sucurez

'Des maus a cestui (me) delivrez,

'Si vus promet pur verité.

528 'Sanz enfreindre la veray voé,

' $\mathrm{Si}$ vus a ceste feiz aydez

'Pur nei succure e travailliez;

'Puis eeste hure mettrai pur veir

532 'Tute una force e mun poeir

'Ke mes travail n'avrez en pose

'De mey ayder pur cele chose.'

Quant seinte Osith aveit dit tant,

536 Este vus une noyse mut grant,

K'ell cele salc est ja levée;

La gent laenz se desree

E haut e bas comunaument

198 Crient e huchent durement,

E vers la chambre vunt errant,

Li un ariere li autre avant.

'A Deu,' funt il, 'li reis u est?

544 'Allas, ke il ne set icest

' $\mathrm{K}$ 'hui en eest jur est avenu,

'Mut se tenist a deceu,

'Si il seust ceste aventure

548 'Kant venu n'i est a dreite hure.'

Ly reis eseute e entent

Lai grant noyse ke funt sa gent,

Coment le vunt tut demandant

$552 \mathrm{E}$ il s'en turne maintenant,

E ver's l'uis de la sale vait,

Pur saver moun quel noise i ait;

E puis k'il lur ad demandé,

556 Delivrement li unt cunté

Cum faitement un poy avant

Un cerfs tut blane $i$ vint eurant,

Ke de la mer tut dreit veneit,

560 Et si curut a grant espleit,

La hors $u$ bien esteit veus,
Par les braches e les seus,

$U$ tut esturent a lur past,

$564 \mathrm{Si}$ cum les chiens rien ne dotast;

E li brachet trestuz cuplé

E li seuz entremedlé,

Li autre chien petit e grant,

568 A fort espleit le vunt siwant.

Ly ke pout unk(es) eheval aver,

Apres s'en est alé pur ver,

E que cheval n'en aveit prest,

572 Tut a pie alé 's'en est.

${ }_{r^{\circ}}^{199}$ Quant li reis ad iceo oy

'Ostez,' ceo dist: 'trop ai dormi :

' $\mathrm{Ca}$, mun cheval delivrement.'

$576 \mathrm{E}$ 'il ne s'est targié nient, Ainz comande trestuz munter,

E sei meimes fet aprester,

Puis est munté [tut] maintenant

$580 \mathrm{E}$ vait le cerf a plein siwant.

Li cerfs s'en vait ou grant bandon,

Li reis apres e cist baron;

Current li chien a grant espleit,

$584 \mathrm{E}$ li cerf est venu tut dreit

Desk'a un bras parfund de mer,

$\mathrm{U}$ ne soleit nuls hom passer,

Kar l'ewe esteit redde e parfunde

$588 \mathrm{Li}$ cerf s'est mis tut dreit en l'unde.

Cel bras de mer li païsant

Castlewade vunt apellant;

Li cerf en l'ewe noe amunt

592 Les chiens anguissus sunt, Crient e funt noise mut grant.

A nou le vunt a plein siwant,

$\mathrm{Li}$ reis en est mut anguissus,

596 Del cerf prendre mut desirus,

Kar unkes ne vit il nul jur

Cerf ne bisse de sa blanchur.

Il vait puignant par eel sablon,

600 Hurte chival de l'esperon,

Coment chevache ne li ehiiet,

En bras de mer parfund se met,

Red e parfund esteit le guez,

604 Devant ceo n'ert unc mes passez

Par home a chival ne a pié,

\section{Cf. $\$ 5$.}

536 Read es vus and compare 1. 905.

542 Cf. $\$ 66$.

556 Cf. $85 \mathrm{II}$.

531 Cf. $\$ 53$ III ii.

537 Cf. $\$ 15$.

554 Cf. $\$ 59$.

vv. 558-626 Subito quidam cubiculum intrasse fertnr qui regi nuntiaret cervum nive candidiorem pro foribus aule undique libere $\left[121 \mathrm{v}^{\mathrm{o}} \mathrm{a}\right]$ discurrere quasi regi et suis omnibus insultaret. Quod rex audiens ceptam voluntatem mox postposuit et cervum cum suis venatoribus et militibus perniciter insequitur.
572 Cf. $\$ 65$.
574 Ostez. Cf. $\$ 59$.
589 Cf. line 185.

592 It is improbable that this line is as it left the poet. The scribe may simply have omitted a member of the phrase as e.g. de siwre (cf. l. 595), or, what I consider more likely, have changed an older reading containing the word setis (e.g. li seus mult...) which had become obsolete (cf. line 566-where the meaning is plain from the context-and the remark of G. Paris in Introd. to Vie de saint Gilles, p. xviii).

601 Cf. $\$ 4 \Lambda$. 
${ }_{\mathbf{r}^{\circ}}^{196}$ Trestut primes l'ad asaié.

L'ure quant sunt li chien passé

608 Estoit li reis parfund en gué;

Mes li cheval iert bon e fort,

Tut sulement se met a port.

Ly cerf a plein en chemin entre

$612 \mathrm{E}$ vunt li chien curant sur ventre,

E li reis vient apres puigniant,

Ses chiens a plein esbaudissant.

Mes li cerf n'est unkes restuz,

616 Dekes Donewiz est dreit veuuz,

Iluec s'est mis en cele mer.

$\mathrm{Li}$ reis se peine del haster,

Mes n'i put unc si tost venir,

620 Pur rien ne put le cerf choisir.

Ne set coment s'en est partiz

Kar de ses oilz est evaniz.

Les chiens esteient tut asoté,

-624 E sus e jus unt regardé.

Ore penst d'autre cerf li reis,

Kar ne verra cesti del meis.

Tant cum li reis vait demorant,

$628 \mathrm{E}$ ou ses chiens le cerf siwant,

Seinte Osith n'ad pas oblié

En quel pour aveit esté.

Ainz dist ke mes ne targera

632 De ceo k'out empensé peça;

Coment ke li plai pust se prendre,

A Dampnedeu se voudra rendre;

Puis en apres la Deu ancele

636 Tuit en secrei a sei apelle

Seint Ecca e seint Bedewin,

E si lur dist le chief enclin:

159 'Seigniurs, ore m'escutez ici,

640 'Pur amur Deu merci vus cri,

'Pensez de mei conseil doner

' En mund ne voudrai plus ester;

'Ne mes suffrir le grant ennui

644 'En cel habit $u$ ore sui;

'Jeo voil ke le veil me donez,

'Pur nule rien me ne targez;

'Jeo l'ay tut prest ici ou mei,

648 'Prendre le voil en bone fei.'

Cil responent: 'Dame merci
'Nus ne l'osum pas fere issi.

' $\mathrm{Kar}$ vus estes joint a seigniur

652 'Coment k'entre nus seit l'amur,

- Par nostre asenz [nus] n'osum pas

' Muer a vus l'abit des dras;

'Mes si vus plest dame suffrez

656 'A ceo purrez venir assez;

'N'osum tel(e) chose uncore enprendre

'Deske en avant bien est d'atendre.'

$\mathrm{E}$ seinte Osith requert assez.

660 Pur Deu amur les ordenez;

K'il facent ceo ke lur ad dist

Mes il li unt tut escundist;

$\mathrm{E}$ quant la dame bien entent

$664 \mathrm{Ke}$ ne volent faire nient;

'Seigniurs,' ceo dit, 'quant est issi

'Deu penst de mei par sa merci.

Plus en apres leva sa main,

$668 \mathrm{E}$ treit le veil hors de son sein,

E sur l'auter offrir le vait,

Puis de sa main vers li le trait,

E sur son chief le veil ad mis,

$\underset{v^{0} \mathrm{~b}}{139}$ E ataché e bien asis;

$\mathrm{E}$ dit: 'Dampnedeu tut puissant,

' Cors e alme ci vus comant,

'Si me gardez pur vostre nun,

676 'Car ne ay d'autre guarisun.'

Seinte Osith ad cest oure en pris

Ke sur son chief le veil ad mis.

Le rei revient ja de chascer

$680 \mathrm{U}$ gueres ne put espleiter,

Corus e plein de maltalent;

A l'us de la sale descent,

Car custume est, bien le savez,

$684 \mathrm{Ke}$ riche home coruce asez,

Kant sa beste aver a failli,

$\mathrm{E}$ il refist tut autresi.

En la chambre est droit alé,

688 La reine a tost demandé,

$\mathrm{E}$ ele vient en son neir veil, Al rei en fremist chacun peil.

L'alme del cors pur poi s'en ist,

692 'Tant s'esniai[e], tant enpouerist,

\section{Omit tut or cf. $\$ 65$.}

633 MS. pus se prenge; pust for peust cf. § 5 II.

vv. 626-78 Quod cernens beatissima virgo ac divina dispensacione hoc actum intelligens, statim velut ovis erepta de ore leonis, sanctissimos pastores et pontifices Eccam [MS. Neccam] et Bedewinum accersivit, propositum eis sue mentis humiliter indicavit, deprecans ut eam sacro velamine cooperirent, si forte virginitatem suam sic protegere posset. Qui votis ejus humiliter et ipsi faventes eam sicut rogaver[at] Christo mox consecraverunt ac sacro velamine cooperiverunt.

665 Cf. $\$ 59$. 687 Cf. Hiatus.

vv. 678-718 Quod cum rex de venacione rediens comperisset, ex intimo cordis affectu dans suspiria graviter doluit, (quia eam sicut proprium corpus dilexit).

691 Corr. n'en ist. Cf. $\$ 53$ Iv.

692 empaorir seems to be rare in verse; Godefroy has no quotations except in prose passages. Cf. $\S 31 \mathrm{f}$. 
E quant regard la cou(e)le bise,

A poy d'effrai le quer n'i brise.

Del cerf li membre k'il tant suivi,

696 Quide ke deable l'en a trai ;

(Mes) quant se parceit de la reine

De rampuner la ne fet traine

Mut la comence a laidenger

700 Des paroles e a tencer,

Coruce e deve a desmesire

Asez li dist, asez li jure,

Dist par serment e manascer,

704 Tut son engin n'avera mester;

${ }_{r_{\mathrm{a}}}^{140} \mathrm{Ne}$ purra pas si enginner.

Mes ele li dist pur nient le fet,

708 Pur nul homme n'ert [ja] defet,

Dekes a Deu s'est si rendue,

Mes ne put li estre tolue;

Ainz voudra meuz la mort suffirir

$712 \mathrm{Ke}$ cest abit james guerpir.

A desmesure grant dolur

En fet li rei e nuit e jur;

Lesse son beivre e son manger,

716 Cum fu jadis acustumer,

Ne put nuls hom a ly parler,

N'en la chambre gueres entrer.

Kant sa dolur a fet asez

720 Ke tut put estre alessez,

Purpense sey a chef de tur

Ke rien ne vaut sa dolur,

Kant veit ne puet estre nuuee,

724 A seinte Osith a gr[a]antée,

Ke remaine tut autresi

En cel albit k'ele ad choisi;

S'il veit ke el estre [ne] peust

728 Ja le congé pur lui n'eiist;

'Dame (ceo),' dist il, 'quant issi est

' $\mathrm{Ke}$ (jeo) ne puis vus tollir icest

'Ceo peise mei estre mun gré,

732 'Or seit a vostre volunté

'Mes quant l'avez issi empris

'Des or n'i ad ell, ceo m'est (a)vis;
'Mes a ceste religion

736 'Covent ke jeo meste mun don

'La vile Chich vus doins issi,

'Kenclovedene tut autresi,

${ }_{100}^{140}$ 'Tut ensement Hodefeld averez,

740 'De ceo faites vos volentez,

' Kar jeo vous bien frai estorer;

' $E$ estre ceo vus voil doner

'De chescun conte e de buron

744 'La secunde fille par non

'De tant cum ad en muu regné ;

'E puis a vostre volunté

'Enscmble ou vus serrunt veléez,

748 ' $\mathrm{E}$ en religiun donéez.'

Si cum il dist issi l'ad fet,

E seinte Osith mut tost s'en vet

Pur sa meisun faire aturner

752 U deit, pur Deu servir, rester.

Aprester fait delivrement

Des offices ceo ke apent;

Les dameiseles sunt inandéez

$756 \mathrm{E}$ de par Deu mut tost velée\%;

Ou seinte Osith remises sunt

E tut guerpi l'onur del mund,

A Deu servunt devotement,

$760 \mathrm{E}$ mut i vivent seintement.

Apres lung tens avint issi,

Cum vus avez asez oi,

Des ces paens Deu enemis,

$764 \mathrm{Ke}$ ja vindrent en cest pais,

De Danemarche lur dreit curs,

Gent hase de pute murs,

$\mathrm{Ki}$ sunt venu par dreit fuire,

768 Pur crestiens par tut destruire,

Li deuz tyranz ki unt mené,

Ynguar e Ubba sunt nomé;

Les crestiens vunt destruant,

${ }_{\mathbf{v}^{\circ}}^{140}$ Chasteus e viles asegant,

$\mathrm{Ne}$ en pernent autre rancun

De crestien for le chef nun.

Cil deuz tyranz pur verité,

776 Seint Eadmund unt decolé,
$694 i$ cf. $\$ 48$.

696 Cf. $\$ 53$ i.

721 Corr. puepensa.
vv. 719-60 Nec tamen illam a proposito suo revocare ausus fuit immo concessit ei ut ipse optaverat castam permanere. Acta sunt igitur hec apud orientales Saxones juxta mare in villa que Chic ab incolis regionis illius nuncupata est, anno dominice incarnationis sescentesimo quinquagesimo tercio. Quam villam rex in possessionem eidem virgini tradens abiit in qua et ipsa usque ad finem vite sne caste perseverando remansit (ubi edificia lapide et latere pulcre constructa habuisse dinoseitur). [Bodley, ibid.]

733 MS. avet.

738-9 I have not been able to identify these names, the former is perhaps a mutilated form of Camulodunum (Colchester).

vv. 761-806 Qua Deo in eodem loco jugiter fideliterque serviente quidam pirate paganis adhuc irretiti erroribue (cum jam yer diversas Anglie partes cedes quasi plures debacchando crudeliter exercuissent), forte illuc applicuerunt, virginemque compertam atque correptam idola blandiciis suadendo minisque terrendo adorare coegerunt. Sed virgo beatissima et blandicias respuit et tormenta derisit. Unde princeps (illius nefandi exercitus) in iram versus capite plecti virginem jussit. 
E puis comencent a errer Par la costere de la mer, $E$ en viles entrent partut 780 E ocient la gent a but, Ardent les eglises a plein, Tuent les prestres de lur mein, Robent la gent, funt envayes,

784 Par tut la u trovent abeies Le feu i mettent de lur meins, Moines ocient e noneins, U k'il viengent, ceo saciez bien,

$788 \mathrm{~A}$ destruire ne lessent rien, $\mathrm{E}$ en lur nef entrent apres, Par icele mer vunt ades, Tant unt nagé, tant unt siglé,

$792 \mathrm{Ke}$ pres de Chich sunt arivé, E puis saillirent sur cel gravier, Le pais vunt tut enchercher, Pur quere cristiene gent,

$796 \mathrm{~K}$ 'a quer heent trop cruelment. Seinte Osith fu le jur alée Si cum esteit acustumée, A une secrée fontaine

800 Quatre meschines ou soi meine, Pur sei laver $i$ sunt alées E li paen les unt trovées; N'entendirent pas lungement

804 Ainz decolent comunaument

$\nabla_{\mathbf{v}^{\circ} \mathrm{b}}^{140}$ Seint Osith ke unt trové ci $\mathbf{E}$ les autres ensemble ou li. Mes seinte Osith tut erranment 808 Son chef entre ses mains prent, Si cum n'eiit anguisse eüe, Tant veit k'al muster est venue;
La fontaine dont (ele) est alée

$812 \mathrm{~A}$ deuz quarenteines de veie E plus est loinz de cel muster A dreite veie u a sentier, $U$ seinte Osith son chef porta.

816 L'us del muster puis en entra De ses mains l'ad ensenglanté Dunt ele aveit son chef porté. L'enseigne fu apert e grant,

$820 \mathrm{E}$ lung tens puis aparissant, $E$ si raveit tut ensement En nostre tens asez [de] gent Ke l'us virent ensenglenté

824 Par ki savum la verité. Seinte Osith n'est unk arestue, Deke al auter est dreit venue, $E$ de son chef i fet present

828 A Dampnedeu omnipotent, A ly del tut s'est comandée Pur ki amur fu decolée, $\mathbf{E}$ puis repeire belement

832 E entre pilers se estent, En presbiterie del muster, $\mathrm{E}$ ses meins comence a drescer. Vers Dampnedeu son creatur

836 Pur ki suffri mort e dolur, [E] torne dreit vers l'orient

${ }_{r^{0} \mathrm{a}}^{141}$ A Dampnedeu l'esperit rent.1 Seignurs ore avez bien oï

840 De la vie seinte Osith ci, Coment pur Deu guerpi le mund $\mathrm{E}$ les richesces k'ici sunt Pur trover joie pardurable

844 Ke a tut dis ert estable,

784 Cf. $\$ 5$ в ii (a).

vv. 807-24 Que decollata se ilico erexit et caput (a corpore longius [121 $\mathrm{v}^{\circ} \mathrm{b}$ ] rotatum) ambabus manibus accipiens angelico juvamine firmis gressibus illud in ecclesiam que in honorem apostolorum Petri et Pauli antiquitus fuerat constructa detulit. (Distat autem locus sue decollationis ab illa ecclesia quasi stadiis tribus. Cumque caput deportans ecclesiam ingrederetur, ostium cruenta manu forte tetegit. Cujus rei signum nostris contemporaneis manifeste apparuit.) [Bodley, ibid.]

vv. 825-56 Hujus igitur venerabile corpus ante introitam chori ecclesie prefate a fidelibus boneste sepultum diu jacuit ubi ejus meritis multa miracula Deus ostendit. (Nam postquam pro fide Christi mortem suscipiendo occubuit atque in ecclesia illa ut dictum est sepulta fuit, sanitates diversarum infirmitatum ibi usque in presentem diem celebrari non desinunt.) Que cernentes pii populi virique sapientissimi cum ymnis et laudibus corpus ejus de eodem loco transtulerunt et in medio choro cum sarcophago honestius posuerunt. (His temporibus contigit mirabilem virum nomine Mauricium Lunduniensem fieri episcopum. Qui cum de virtutibus et miraculis sancte virginis audisset atque eam in sua parochia esse cognovisset, locum hnmiliter frequentavit, virginemque dictis et factis decenter honorificavit. Cui quoque inter alia placuit ut corpus illius de loco in quo jacuit levaret et in loco eminenciori scilicet post majus altare honestissime poneret. Quod cum devotissime fieret atque tandem venerabili corpore ad votum episcopi positum esset, balsamum quantum habuit sibi jussit afferri atque allatum supra sacrum corpus gaudenter effudit et effundendo ampullam vitream in qua fuit coram omni multitudine que advenerat guttatim excussit. Sed hanc ampullam coram omnibus ut dictum est evacuatam [122 $\mathrm{r}^{\circ}$ a] alio rursus tempore satis meliori balsamo usque ad summam Dei munere repletam episcopus recipere promeruit. Nam non multo post contigit quendam ministrum ejusdem episcopi adeo infirmari ut nec loqui nec aliquid movere se posset sed quasi animam statim redditurus extensus jaceret. Quod cum episcopo nuntiatum fuisset ampullam predictam sibi afferri jussit ut saltem 
Trovée l'ad finablement

Sachez ke nen dotum nient,

Kar Deu le rey de majesté

848 Apertement l'ad ci mustré

Par miracles gentils e granz

Ke pur ly fet aparissanz,

Fort serreit tut a acunter

852 Mes partie volum mustrer,

$\mathrm{U}$ vus porrez tres bien oir

K'a Dainpnedeu fet a servir,

Ky leaument le servira

856 Sanz gueredon ne s'en irra.

Cum nus avum pur verr oy,

$\bigcup$ Acune feiz avint issy

Ke grant tempeste out en la mer,

860 Horriblement la fist emfler,

Jeter guages e verser undes,

Lcides, horribles e parfundes;

$\mathrm{Ki}$ dune i furent, bien vus jur,

864 N'esteient pas del tut seur.

En grant loure de la tempeste

K'en mer feseit si grant moleste,

Par la tormente sunt chaciez

868 Nefs estranges e travaillicz;

Une hore aval, un autre amunt,
Tant cum tut dreit venues sunt

${ }_{r 0}^{141}$ En cel havene puis al d[e]rein

872 A seinte Osith le plus prochein.

Les mariners i unt ancré(e),

Sigle abatu e bien teoldé(e),

Ilokes vunt cum est custume

876 De tens attendent suautume,

En havene sunt bien lungement

Pur atendre le prospre vent,

E vunt tel hore est a la terre

880 Pur vitaille e garison quere;

A seinte Osith revunt suvent

A oreisuns ou autre gent.

Un jur i vunt les mariners,

884 I y envoisez les juvencels, Si cum esteient custumer

E pur orer vunt al muster.

A ceo k'aukes i unt esté

888 E sus e jus par tut alć,

Li uns d'eus cum esteit alant,

Trove un marbre gisant

Pres deuz espaumes de lungur,

$892 \mathrm{E}$ pleine paume out de laur.

E cil la prist delivrement,

Si l'enporta tut belement,

F si comence a purpenser

896 K'en son pais la vout porter,

ejus ablucionem super linguam infirmi distillaret si forte gustu illius liquoris aliquid verbum confessionis peccatorum edere posset. Mira res. Nam eadem ampulla episcopo allata optimo halsamo Deo munere plena inventa est. De quo cum infirmus parum gustasset, mox sanitate recepta surrexit et letus quo volnit deambulavit, episcopo et omnibns qui aderant valde admirantibus et Deum in sancta virgine Ongitha laudantibus. Translatio autem hujus gloriose virginis tercio nonas octobris sub Mauricio venerabili episcopo ut prefati sumus facta est et altare ad capnt ejus endem die dedicatum.) [Instead of the passage in brackets, MS. Lansdown has only: Deinde Mauricius Londoniensis episcopus corpus ipsius levavit et post majus altare posuit.] Cujus festum nonas Ootobris manifestamus esse colendum quia eodem die pro Christo agonizans mortem temporalem sabiit et in gaudium sempiternum cum corona martirii gandenter intravit. Translatio vero ejus jubente domno Ricardo successore Mauritii, in crastino id est octavo idus Octobris celebratur quia ut isdem venerabilis episcopus dicehat inconveniens erat tamprope ante illius martirii festum translationem ejus celebrare. (O quot et quantis miraculorum signis hec bestissima virgo quanti sit meriti non solum suis compatriotis verum extraneis manifestata est. Sed multis propter fastidium legencium omissis unum ad memoriam referre delectat.)

851 Cf. $\$ 59$.

vv. 857-86 Tempore enim prefati pontificis Ricardi quidam [122 $\left.\mathrm{r}^{\circ} \mathrm{b}\right]$ theutonici cum quadraginta navibus alleciis onerstis sicut per singulos solent annos de Gernemutha redierunt et ad portum sancte Osgithe qui lingua incolarum Westmutha vocatur omnes tandem convenerunt. Qui ex singulis navibus elemosinam collectam more solito ad ocolesiam miserunt ut virgine pro eis intercedente ventum prosperum quo repatriarent recipere meruissent.

874 This refers to a Scandinavian custom of erecting an awning over the deck when in port called tialz; in St Gilles we find Funt un tialz desus le tref, E puis s'en issent el graver... 930-1; the word is Old Norse tjald (Germ. Zelt; Eng. tilt). Cf. $\$ 5$ в ( $\mathrm{x}$ ), \&59.

883 Cf. $\$ 3$. The rime words were doubtless in the original, marinel; juvencel.

890 Corr. a terre gisant. Cf. Latin text, or 1.990.

vv. 887-904 Cumque oblatione facta quidam illorum qui eam attulerunt in porticu anstrali consedissent ęt ad invicem de amenitate et situ loci loquerentur, contigit forte quoddam frustum marmoris ibi velut neglectum aut projectum in pulvere jacere. Quod cum quidam ex illis latenter rapuisset, secum sociis ignorantibus illud ad navem portavit. 
E pense bien k'en son muster

Al pais domer avera mester.

Le marbre porte si s'en vait

900 [De]vers la marine tut dreit, Entre sa nef de meintenant,

E del marbre ne fet semblant, A compaignon mot ne sona,

${ }_{\mathbf{v}^{0} \mathrm{a}}^{11}$ Kar plai n'en tint, bien le mustra.

A tant es vus si unt [bon] vent,

Dreit e portant a [lur] talent,

Drecent lur mast e cordes tendent,

$908 \mathrm{E}$ le wydas par tut amendent,

$\mathrm{E}$ puis unt trait lur sigle amunt,

Ancre sakent e si s'en vunt.

De cele nef oir porrez

912 U li marbre fu enz portez,

Kant li autre vunt herneschant,

E lur aferes adrescant,

E s'aturnent e lit e ci,

$916 \mathrm{E}$ cil refunt tut autresi;

Mes kant unt le mast bien fermé,

Ancre saké et veil levé,

E les autres par tut s'en vunt,

920 Cum cil ki vent a talent unt,

Unk cele nef ne fist semblant,

K'en ewe fust ne tant ne quant,

Ne pur force, ne pur saver,

924 Ne la porrunt del liu mover.

Les mariners levent lur main

Chescun d'eus se seignie a plein,

Dient entre eus: 'avez veu

928 ' Itel merveillie ne unkes fu;

'Dunc n'avum nus le vent portant

'Cum cil autre ki vunt devant

'Le governail bien ataché

932 'De gurderis ancre saké

' $\mathrm{E}$ drecié mast, sigle amunt trait

' Ke deit ke nostre nef ne vait?'

Puis comandent les compaignons

$936 \mathrm{~K}$ 'il augent tut a avirons, ${\mathbb{\mathbf { v } ^ { 0 }}}_{\mathbf{1}}^{\mathbf{1 4 1}}$ De totes parz pur asaer

Si rien ne vausist lur nager,

Enteims pur mut estre grevé

$940 \mathrm{Ke}$ del havene seient geté,

Les juvenceus pruz e legier(s)

Se peinent mut del efforcer(s),

$\mathrm{E}$ chescun d'euz i met sa main,

944 Des avirons ferent a plein.

Mes plus semblant ne fist la nef

Ke maison fet le cuchetref ;

Bien la porunt conduire ariere

948 Mes avant en nule manere;

Costeant veit e en belif

Dreit cum cheval ke est restif.

Kant cil veient ke n'ad mester

$952 \mathrm{Ne}$ lur sigler ne lur nager,

Parolent en estreitement

Cume cil ki sunt mut dolent.

'Scignurs,' fet l'un, 'ça entendez,

956 'Nus sumes mal bailli assez

' $\mathrm{E}$ cel veurn tut a estrus

' Ke le ire Deu vient sur nus,

'Pur nos pechez bien le savum

960 'Cest encumbrer en mer avum;

'Criums merci cher compaignons,

' Pur Deu le veir e pur ses nons

' Ke cil ke se savera copable,

964 'Encumbré de peché del deable,

' Ke il put sei fere confes

'De penitance prendre apres

' Ke Dampnedeu merci en ait

968 'De ceo dunt il ad mesfait;

'Pur Deu seigniurs ki ke ceo scit,

${ }_{\mathrm{r}^{\circ} \mathrm{a}}^{142}$ ' $\mathrm{Ke}$ conisant seit ore endreit,

' Ke pur un sul e sun peché

972 'Ne seum tut ci perilié;

' Kar s'il n'i ad amendement

' Ci demorum finablement.'

A ceo (ki l')unt longement parlé,

976 E entre sei chescun pensé,

906 Cf. St Gilles: Kar ore vente a lur talent 1032.

vv. 905-24 Quo facto vento surgente prospero anchoras subtra[h]unt vela suspendunt et versus patriam omnes cum gaudio volucri cursu tendunt, preter unam navem que velut in terra sic in aqua juxta litus fixa remansit.

vv. 925-50 Nec tamen naute navis ejusdem tardius ant pigrins aliis anchoras subtraxerant, immo velum usque ad mali sumitatem trahere studuerant. Sed nec aliqua instrumenta nec velum Deo jubente suum tunc poterant officium habere. Naute itaque dolentes et causam penitus ignorantes per navem discurrunt, funes expediunt, contos exponunt, remos deiciunt, clamando pellunt, trahunt nec quicquam proficiunt.

934 'How comes it about that...' cf. $\$ \$ 53$ r, iv, 59.

945-6 The sense of these two lines is obscure and cuchetref $<*$ colloca trabem is ap. parently not known elsewhere. I am inclined to think it is the awning referred to in line 874 footnote. The meaning would appear to be that the ship was no more like a ship than a 'cuchetref' is like a house. Cf. $\$ 59$.

vv. 951-98. Fessi ergo considunt, peccata plangủnt, vota vovent, emendationem vite Deo et sancte Osgithe omnes flebili voce promittunt. In terea isdem homo de quo superius mentionem fecimus in medium prostitit, se graviter peccasse dixit et marmor quod abstulit omnibus ostendit.

961 Corr. compaignon.

962 Corr. son non, cf. $\S 43$ iii. 
De ses mesfez de ces pechez.

A tant s'est drescé sur [ses] piez

$\mathrm{Ky}$ enporta le marbre issi.

980 'Seigniurs,' ceo dist, 'pur Deu merei

'Endreit mei voil primes parler

'Le men mesfet ne voil celer,

'Si mesfet deit estre nomé.

984 'Ceo k'en mon quer ay recordé

'Poy de chose est, me[s] nekedent

'Dirray le vus tut erranment.

'A seinte Osith estoie aléz

988 ' $\mathrm{Ou}$ autre compaignons asez,

'En son muster u jeo entray,

' Un marbre petit i trovay,

'Si l'enportay par aventure

992 'Par geu le fis e enveisure,

'Ci l'ay porté, ici l'ay miys

'N'enquer celer ceo ke en fis,

'Or vus en ay le veir gey

996 'Beaus compaignuns pur veir vus di,

'Si de ceo ay vers vus mespris,

'Faire en voudray tut vostre avis.'

Quant l'unt oï funt [mut] grant bruit

1000 A une voiz s'escrient tuit :

'A Dampnedeu graces rendum,

' Kant tant enquis suvaus avum

142 'Or n'i ad en for retorner

1004 'Le marbre ou uus volum porter,

'A seinte Osith tut dreit alum,

'E Deu e ly merci crium,

'Rendum le marbre a son nuster,

1008 'Mut covient iluc Deu prier

' $\mathrm{K}$ 'il nus pardoint icel peché

' Ke mes ne seum travaillié,

'Cum nus avum esté devant,

1012 'Kar ci sumes trop sujurnant.'

Sicum unt dit issi le funt,

Issent de nef e si s'en vunt;

A seinte Osith sunt dreit alé;
1016 Kant en muster furent entré, Sachez ne sunt unkes restu, Dekes al auter sunt dreit venu, A genouilliuns iluec euché,

1020 Crient merci de lur peché, Le marbre $i$ unt offert e mis

E Dampnedeu sovent requis E seinte Osith sa chere amie

1024 Mes ne seient en tel baillie, Ne travaillié ne malmené

En havene u tant unt demoré;

E communaument voé unt

$1028 \mathrm{Ke}$ checun, tant cum viverunt, Lour offrende ferunt porter A seinte Osith e presenter, Ke Dampnedeu omnipotent

1032 A aler lur doint sauvement. Al pople k'il unt iluee trové Curn lur avint unt cunté, Permunt congé e si s'en vunt,

${ }_{\mathrm{v}^{\circ}}^{142}$ E en lur nef puis entré sunt, Levent sigle hastivement; Si tost cum i feri le vent, La nef se torne a tel randon,

1040 Come colum devant faucon, Deu fist iluec [tres] grant vertu E miracle tres bien seu, Kar cil s'en vunt par cele mer,

$1044 \mathrm{E}$ poesté unt de sigler Les nefs ke peca sunt aléez $\mathrm{E}$ loin en mer avant passéez, Cil ki tant esteient ariere,

1048 Ne sai coment n'en quel manere Fors cum Deu le vout en maesté, Lour compaignons unt ja passé; Errent e vunt siglant afort,

1052 A tant vienent plus tost a port; $E$ les autres passent de tant Cum ariere esteient devant. N'at cil saciez k'il n'eit oy,

986 The scribe writes now erraument and now erraument. I have chosen the former orthography in each case.

vv. 999-1032 Quod etiam omnium hortatum ad ecclesiam referre festinavit. Navis vero interim quasi hominem $\left[122 \mathrm{v}^{\circ}\right.$ a] expectans Dei jussu ibidem donec ille rediit immobilis permansit.

1033 Cf. 866.

1034 Cf. $\$ 64$.

vv. 1033-54 Que[m] redeuntem recipiens velut a vinculis soluta prosilivit et post precedentes ocior volucre advolat. Et licet a conspectu illius alie jam longe abierint, hec tamen citius omnibus portum desideratum ingressa est. Ex quibus postmodam quidam in Anglia reversi coram altari sancte Osgithe omnibus qui aderant ista subtiliter sicut gesta fuerant narraverunt et quod ille vir per quem hec acciderant Antwerpensis [gloss in a beautiful xiii century hand: Andwerpia villa est in Brabantia et portus maris] natione et Theobaldus nominatus sit fideliter intimaverunt. Pro quibus omnibus Deum laudemus ipsamque virginem humili prece deposcamus nt nobis peccatoribus apud Deum nostrum Jhesum Christum subvenire dignetur qui vivit et regnat cum Deo patre in unitate Spiritus sancti Deus per omnia seculorum secula, Amen. Explicit vita vel passio beate Osgithe virginis.

1049

1051 This should perhaps be written au fort, common in the meaning enfin. 
1056 Coment la nef siglat issy,

Ke mut ne seit esmerveillié,

Kant enquis unt e cerché

$\mathrm{E}$ entendunt la verur,

1060 Mercient Deu le creatur,

E seinte Osith la gloriuse,

Martir e virgine preciuse,

Pur ki Deu fist si grant vertu

$1064 \mathrm{Ke}$ apertement le unt veu.

utre miracle apres orrez

A De seinte Osith si l'entendez. Une femme contraite esteit

1068 Vers Hereford de Wales dreit,

${ }_{\mathrm{v}^{\circ} \mathrm{b}}^{142}$ Ke ne poeit en nule baillie

De li mover sanz aïe,

Des membres ne se poeit ayder,

$1072 \mathrm{Ne}$ al lever ne al cucher,

De ses membres tut dis estoit

En grant anguisse e grant desdroit, Asez ala par Engleterre,

$1076 \mathrm{~A}$ oreisuns les seinz requere, Par tut u ele oï aveit

$\mathrm{Ke}$ Dampnedeu vertu feseit, $\mathrm{Ne}$ puet uncore estre oie

1080 Kar Dampnedeu ne voleit mie.

Kant alé tant aveit par tut

Ke ses amis esteient mut

De li partir ja travaillié,

$1084 \mathrm{E} \mathrm{au}$ derein mut ennuié.

Ne saveit mes a requere,

- Seint ne seinte en Engleterre,

$U$ ne aient ja fet venir

1088 Fors seint Edmund le bon martir.

Ia chaitive fu (mut) anguissuse,

$\mathrm{E}$ de garrir mut desiruse,

Tant ad prié, tant ad requis,

$1092 \mathrm{E}$ ses parenz e ses amis;

A grant peine granté li unt

De li porter a seint Edmund.

Sa suer par non ke mut l'ama

$1096 \mathrm{E}$ par terre tant la mena,

Ore a derein tut ensement

Icest labur pur li enprent.

Si l'aturnast a meuz ke poeit

1100 Vers seint Edmund s'en vunt tut dreit.
Tant errunt petites jurnées,

${ }_{r^{\circ}}^{143}$ Cum furent bien acustumées,

Ke nequedent a chief de tur,

1104 Le seurveillie devant le jur

Seint Michel venues $i$ sunt

E la contraite porté unt

A grant travail dekes al muster

1108 Pur faire la iluec veillier.

Mes kant vint tut dreit al vesper

Li secrestains la fait oster,

E estrussé li ad mut bien,

1112 Suffrir ne vout pur nule rien

K'ele la nuit seit al muster,

$\mathrm{Ne}$ pur orer ne pur veillier,

Oster la fist demeintenant

$1116 \mathrm{E}$ cele en fet doil mut grant.

Quant de l'eglise esteit ostée

A ceus a dit ki l'unt portée:

'Pur Deu amur merci vus cri,

1120 ' Ne me portez [tres] loinz de ci, 'Metez mei ci dehor's cest us

'Lessez mei iluec, ne vus quer us

$\mathrm{E}$ cil refunt tut autre tel

$1124 \mathrm{E}$ puis se vunt a lur ostel.

Tute la nuit iluec remaint

La contraite ke mut se plaint,

E crie Dampnedeu merci

$1128 \mathrm{E}$ seint Edmund tut autresi,

Ke puisse aver amendement

De mal ke la tient forment.

A meuz ke sout vers Deu orat,

1132 Tute la nuit iluec veilliat,

E pres del us s'est ajustée,

Tut dreit cum vint en l'ajurnée

${ }_{\mathrm{r}^{\circ} \mathrm{b}}^{143}$ La cheitive ke tant i crie

1136 Un petitet s'est endormie.

De hors cel us u ele jut

Un hom en blanc li aparut:

'Diva,' ceo dit, 'ki es tu ci

1140 ' $\mathrm{Ke}$ tute nuit me cries si

'Ke demandez, ke vus aveir?'

Cele li dist: 'sire pur veir,

'A Dampnedeu jeo vinc ici

1144 ' A seint Edmund tut autresi,

' Ke jeo requer pur son [seint] non,

' $\mathrm{Ke}$ il m'envoie guarison.

'Mut ay par Engleterre erré

1148 ' $\mathrm{E}[\mathrm{n}]$ oreisuns pur ma sancté,

1058 Corr. encherché as in 1. 794.

1059 The tense and form may be original (cf. $\$ 55$ i) but it seems a scribe's error for entendu unt; cf. 1.1270.

1069 Omit $K e$.

1070 Both here and at 1. 1176 nule (nul) may have been omitted.

1074 This would appear to be a scribe's error for destreit. Cf. Marie de France : La dame conut bien le pleit. Mult est sis quers en grant destreit [Guigemar, 801-2] but the word may have existed.

1085 Requere has no object in the phrase, qui may be inserted.

1099 Corr. si l'aturne? Cf. 1. 1131.

1116 Cf. \$ on Hiatus. 1130 Corr, de cel mal.

1108 Cf. $\$ 48$.
1141 Vus $(<v o l e s), \quad$ Cf. $\$ 25$. 
'Mes ne oi pas dekes a cest jur, 'Ci sui venue a chief de tur.' Il li respunt demeintenant

1152 'Uncor t'estuet aler avant; ' Kar Deu n'ad pas purveu issi

' Ke guarisun eies ici.

'A seinte Osith t'estuet aler,

1156 'Iluec veillier, iluec orer,

'Kar Dampnedeu t'ad destiné

'En icel liu aver sancté.'

'Sire merci,' fet la contraite,

1160 'Icest respons trop mei desheite,

' Kar chier sire, ceo sachiez bien,

'De seinte Osith ne say jeo rien,

'Ne sai u est ne en quel terre,

1164 '[Ne] seinte Osith ne say u quere,

'Unk mes de li n'oy parler'

'E si resui lasse d'errer,

'E cil ke tant porté[e] m'unt

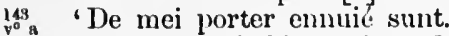

'Avant de ci, bien sai en fin,

'Voie ne sevent ne chemin'

Cil li respont: 'si demandez

1172 'A gent ke ci demain verrez,

- Aucun troverez ell cest entrée

' Ke vus enseignera lir veie;

'Iluec guarrez cum vus ay dit

1176 'Puis en alez sanz respit.'

E cele li dist: 'sire merci

' $\mathrm{Ki}$ estes vus que parlez ci?'

E il respunt mut humblement:

1180 'Seint Edmund me nomment la gent.'

La contraite mut en pensa,

Puis l'endemein quant gent trova

K'esteient a cel us entrant,

$1184 \mathrm{Si}$ lur demande demeintenant

Ver seinte Osith la dreite veie,

Ke pur Deu seit asenseic,

Tant a enquis e demandé

1188 Le dreit chemin li unt mustré,

Tant ad parlé a ses amis

Ke il ja sunt en chemin mys;

Vers seinte Osith la funt porter

1192 Vint jurs i mistrent al aler,

$\mathrm{E}$ travaillient se a merveillie

$\mathrm{Ke}$ venu(e) i sunt dreit la veillie

De seinte Osith kant est la feire

1196 Funt la contraite al muster trere, Dreit a hore porter la funt

Kant li chanoine a vespres sunt,

A ceo k'esteient haut chantant
1174 Cf. $\$ 15$.

1177 Cf. § 5 в $\mathrm{r.}$

1201,1215 Cf. $\$$ on hiatus.

1231 Cf. $\$ 5$ B Ir.

1243 The usual form is nient (cf. $\S 18$ and line 1392), we take nuient as an Agn, form

1176 Cf. 1.1070.

1186 Corr. Kele....

1220 Corr. serur...port. Cf. $\$ \$ 43$ iv., 54 i.

1240 Cf. 1.1250.
1200 Cil vierent ci demeintenaint,

${ }_{v^{\circ} \mathrm{b}}^{14}$ Ke la cheitive unt portée

E einz al muster avalée

Puis [en] vunt al us del chancel,

1204 Acenent les seigniurs mut bel,

K'il unt plus pres del us trové,

Requis li unt pur l'amur Dé

Ke la contraite k'unt portée,

1208 A seinte Osith si longe veie,

K'a sa fertre l'osent porter,

Ke tote nuit en puisse orer;

Ele est a seinte Osith par non

1212 Venue par avision.

Tant unt requis, tant unt parlé

Ke li seigniur li unt granté;

la contraite est remuée

$1216 \mathrm{E}$ a la fertre amunt portée.

Sa suer ke l'ad portée tant

En haut se crie maintenant

- Ma dame seinte Osith merci,

1220 'De ina suer ke vus porte ci,

'Pur ki me sui tant travaillie,

' $\mathrm{E}$ sufferte ay mainte haschie;

'Portée l'ai e sus e jus

1224 'Tant sui lasse ke ne puis plus

'Or la vus ay ci fet porter,

'Avant de ci ne say aler,

'S'ici ne puet estre amendée

1228 'Ja n'iert pur mei avant portée.'

E sa suer l'ad iluec lessée

Devant la fiertre dreit cuchée,

E cele remaint la nuit issi,

1232 E Dainpnedeu crie merci, E seinte Osith [alsi] par non

${ }_{r^{14}}^{14}$ Ke li trameste garison.

Puis unt a matines soné,

$1236 \mathrm{Li}$ chanoine sunt assemblé

Lur matines vunt comencant

$\mathrm{E}$ mut a tant les vunt chantant

Cum a tel feste estoit raisun;

1240 Kant vint a la sime lescun

La contraite geta un cri,

$\mathrm{E}$ pleint e gient e plure si

Ke nui[ent] estoit escuter;

$1244 \mathrm{Um}$ la (co)manda assez cesser

Ke ne desturbast le servise

Mes ele ne pout en "nule guise

Al comandement garde prendre,

1248 Kar ailliurs aveit a entendre;

Ne put son cri amesurer

Le sime respons vunt chanter

Regnum mundi li ordiné,

for noiant, a variant of nient. 
1252 Tut dreit cum sunt en vers entré, La contreite s'escrie tant,

Les nerfs li vunt tut crus[i]sant, Deu reclaime omnipotent

$1256 \mathrm{E}$ seinte Osith tut ensement; Kant longes estoit travaillie Sur piez se est a dreit drescie, $\mathrm{E}$ ver le auter veit tut dreit,

$1260 \mathrm{E}$ loe Deu a grant espleit, E seinte Osith sa chiere amie, Par ki sa preiere est oïe. A chanoines cuntat e dit,

1264 La grant vertu ke Deu li fit, Tut par ordre lur ad cunté, Coment par terre aveit erré, ${ }_{r^{0} \mathrm{~b}}^{144}$ Coment aveit ailliurs failli, 1268 Com[ent] Deu l'ad [a]dresciée ci. Kant li chanoine e li seigniur Entendu unt bien la verrur, Tut loent Deu omnipotent, $1272 \mathrm{E}$ comencent mut hautement Te Deum laudamus par tut; Par cel muster demandent mut Quel noise seit ke la munt funt;

1276 Tut a un frus curru i sunt Deu comencent mut a loer, $\mathrm{E}$ funt les seins par tut soner, Kant la contreite bien entent

$1280 \mathrm{Ke}$ guarie est finement,

E mes ne sent de ses dolurs, Venue est devant les seigniurs, E si lur dit; 'seigniurs merci,

1284 'Dampnedeu m'ad guarrie ci

'E seinte Osith (la) vostre avoée,

'L'amie Deu verraie esprovée,

'Mut aim cest liu e mut l'ai cher

$1288^{\prime} \mathrm{U}$ Deu m'ad si fet adrecer,

'Si pur Delı me voilliez tenir, ' Ja nul jur ne voudrai partir,

'En icest liu finablement,

1292 'A Deu e seinte Osith me rent.' E li seigniur pur l'amur Dé Ke ele remaine unt gr[a]anté. Cele est remise e mut se peine

1296 Trestuz les jurs de la semeine, A plus k'ele puet travaillier Entur l'uveraine del muster; Penible est en $m u[t]$ de manere, $\underset{\mathbf{v}_{\mathrm{a}}}{144}$ Nomeement de trere piere,
E el k'ele puet a cele eglise, Longes remaint en cel servise. Mes deables est mut enginius,

$1304 \mathrm{E}$ de tut biens trop envius, Kant Deu a fet ses granz vertuz, E ses miracles bien veuz, Pur seinte eglise enluminer

$1308 \mathrm{E}$ ses amis reconforter;

Peise deable estrangement E se efforce peniblement D'esteindre ceo pur verité

$1312 \mathrm{Ke}$ Deu ad si enluminé. Dieble n'ad unkes envie, Ne de peché ne de folie, De mesfet ne de traisun, 1316 N'ad envie si de bien nun. La vertu k'est par Deu venue Mut vot turner en fanfelue, Pur fere mescrere la gent

$1320 \mathrm{E}$ ci si fist tut ensement.

Un lur vassal de la maisun, Godwine l'esquieler par nun, Tant ad forment dieble enticé

1324 Empeint a fere malvesté, $\mathrm{Ke}$ a la femme s'est ajusté Tant a requis, tant a parlé $\mathrm{Ke}$ il sunt tut a un voleir

1328 De fere la folur pur veir ; Kar nature est bien le savez Ke femme est frele chose assez Tost ad le quer asis en glu, 1332 Tost ad un mal conseil creu, $\mathbf{v}_{\mathbf{v}^{\circ} \mathbf{b}}^{144}$,Tost ad lessié [la] dreite veie, Tost est en la folur entrée, Tost ad lessé bien e honur,

1336 Tost se prent a la folur. Ceste tut ensement le fist, Lessa le bien e le mal prist, A lur folie unt liu trové,

1340 La nuit kant furent asemblé

En lit trestut primerement E li fous out fet sun talent, Seinte Osith pas ne vot suffrir

1344 Cest overaine endormir $\mathrm{Ne}$ voleit pas pur verite, Iceo mesfet estre celé; L'un pié de la cheitive prent

1348 Sur l'autre dreit en croiz l'estent, Si forment l'ad iloukes mis,

1254 Since $s=88$, ef. $\S 33$, an $i$ could easily be omitted, cf. $\S 59$.

1255 Cf. $\S$ on hiatus.

1275 The woman had been carried up to the shrine, 1. 1216.

1278 Seins (<sanctus) is frequent in Ofr. in the sense of bell, especially to call people together; cf. s. v. sein 2: Godefroy, vii, $365 \mathrm{c}$.

1280 Corr. finablement as in 11. 974, 1291.

1318 Cf. $§ 59$.

1322 This form is quoted by Godefroy under escueillier the scullery, and also as here a sculleryman. Cf. § 59 .

1324 Empeint $=$ urges.

1336 The sense demands $E$ tost. 
Cuché e joint e ferme asis,

Ne puet de luec plus estre ostez

$1352 \mathrm{Ke}$ s'il de clous i fust fermez.

Kant la cheitive iceo entent,

Plure des oilz e gient forment,

E grant dolur comence a faire,

$1356 \mathrm{Ne}$ puet l'un pié de l'autre trere.

$\mathrm{Li}$ pautener quant l'entendi,

Mut s'esmaie tut autresi,

E par matin est tost levé

$1360 \mathrm{E}$ as seigniurs tut dreit alé

As piez lur chiet si lur ad dit:

'Seigniurs entendez un petit,

'Pur nient le vus irrai celant,

1364 'Assez le saverez en avant, 'Meuz le vus voil dire en secrei

${ }_{f^{\circ}}^{145}$ ' K'autre le vus die de mei;

$r^{\circ}$ 'Tut ay en tel manere overé,

1368 'Contre raisun e contre Dé,

' $\mathrm{E}$ avenu est tut issi

'Pur Deu amur seigniurs merci ;

'Trestut issi sui contenu

1372 'Vers Dampnedeu e encorru.'

E li seigniurs demeintenant

La verité vunt enquerant,

L'aventure k'est avenue

1376 Trestut issi est reconue.

A rien ne muntast le celer

Semblant n'i veient demander.

La elheitive ne puet pur veir

1380 Del lit pur nule rien moveir, Les piez li sunt si traversé Cum s'il fuissent des clous fermé, Les seigniurs en sunt anguissus,

1384 Sur tute rien e doler'us,

Al muster vunt [tut] erranment

Requerent Deu communaument, E seinte Osith lur dame ehiere,

$1388 \mathrm{~K}$ 'ele ne souffre en nule manere, Ke sa ancele seit si baillie, Ne al sicele si forment hunie. Requis unt ententivement

1392 Mes tut iceo ne lur est nient. Kar n'amende en nule manere, Pur oreison ne pur priere; Contreite i vint premerement

$1396 \mathrm{E}$ contreite rest ensement. Mut longement remaint issi, Tote contreite cum vus di,

${ }_{r^{\circ} \mathrm{b}}^{145}$ Dekes li pauteners morut

$1400 \mathrm{~K}$ 'en tel manere la decut; Deu fist iluec mut grant vertu Miracle apert e bien veu,
Le jur ke il fu deviez

$1404 \mathrm{E}$ par la mort del siecle alez, La nuit apres [tut] erranment Vint seinte Osith verrayement E la chaitive deliat,

1408 De tut en tut la deliverat, Les piez li ad desseelez, $\mathrm{E}$ quitement desencumbrez; $\mathrm{E}$ cele est par matin levée

1412 E al muster tut dreit alée, E loe Deu omnipotent, E seinte Osith tut ensement. Tut dis remaint en la maisun $1416 \mathrm{E}$ sert ou grant devotiun.

ssez avun oy sovent

A Par reprover dire la gent Ke d'estriver contre aguilion

1420 N'avient a nuli si mal non. Prendre en poum example assez I'un miracle ke ici orrez $\mathrm{Ke}$ Deu pur seinte Osith mustra

$1424 \mathrm{Ki}$ garde en prent, bor le verra. Kar bel se repent de sa folie Ke par autre se chastie, E cil d'assez trop tart se repent

1428 Sur ky le flael Deu decent; Kar Dampnedeus est mut suffrant, E lungement vait deportant, Mes puis quant il fert au derein

${ }_{v^{\circ} \mathrm{a}}^{145}$ Sachez k'il ad mut dure mein; E il refist tut autresi, Seigniurs cum vus orrez ici ;

De le eveske Ricard (essample) dirum

1436 De ceo dunt vus tuché avum, Le tiers a Londres se aveit, Puis cil ke l'abbeie de Chich fundeit,

E a seinte Osith chanoines mist,

1440 Teres e rentes aset $i$ conquist, A cist Ricard le ben ne plout Ke son ancestre a liu fet out. Un jur avint ke fist mander

1444 Ses menestraus e assembler $\mathrm{Ke}$ soleient ses plais tenir, E danz Williame avant venir Son seneschal i fist par non, 1448 De Wokindone aveit surnon, $E$ en ki se fieit mut enfin Ou un Nichole e Rad'patin ; A Dakintone (co)manda aler

1385 Here and at 1.1405 corr. [tut] as in 1.1610.

1405 Cf. footnote to 1.1385.

1403 Cf. $\$ 17$.

1425,1427 Omit se, $\$ 48$ A.

1438 For fundeit cf. $\$ 55$ iv, for a comment on this line of. $\$ 71$.

1450 The apostrophe is in the MS.

1451 The sense seems to be: to forbid the ecclesiastics (cf. 1l. 1439-40) to have possessions at Chich and to take all the profits for his own use. 
1452 De luec oveskaus pur veer Ke a Chic [ne] deusent poseer; E a son eus tut apruer.

E cil s'en vunt a grant espleit,

1456 A Dakintone vienent tut dreit, Iluec tienent a fort estur Les plais l'eveske lur seigniur; En icel tens ke cil veneient

1460 A Chic chanoines mis esteient, Ke Deu serveient humblement E seinte Osith tut ensement; De terre aveient environ

1464 Dunt pussent vivre a fuison,

$\underset{\substack{145 \\ v^{\circ} \mathrm{b}}}{146}$ Estre l'aport de lur autel

K'il aveient qui d'un qui d'el Deu serveient en leauté

$1468 \mathrm{E}$ furent de grant charité.

Le seneschal a Dakintone vint Les pleiz l'eveske forment tint, D'iluec purveit queus enverreit

1472 A Chich kar ces fors mettreit Ki par les chanoines mis est[e]ient ; Oveskeus mestreit ke dureient A li acuntes; l'esveske out voil

1476 Kanoines oster de cel soil; $\mathrm{Ne}$ voleient en nule manere Oir requeste ne preiere, N'entains suffrir hum[e] parler

$1480 \mathrm{Ne}$ terme ne respit doner; Mes si cum lur est comandé Issi en ad del tut overé. Quant les chanoines l'unt entendu, 1484 Mut sunt dolent e irascu,

A Deu s'en vunt pleindre erranment,

E a lur dame tut ensement

Seinte Osith ke unt servi tant,

$1488 \mathrm{Ke}$ del surfet seit eus vengant. K'ele deust bien a lur avis Defendre les de lur enemis, A sa fiertre vienent errant,

1492 L'ymage ostent tres estant, Hors l'us l'eglise l'unt posé Cume pur prendre son cungé. Le fiertre covrent d'une here,

1496 Ceo signe ke de joie volent trere, $\mathrm{Ne}$ volent (seinte) Osith plus loer $\underset{r^{\circ} \circ}{146}$ Kar par semblant le liu n'ad cher,

Ou lermes o ou plaintes funt

1500 Asez saver ke il au quer unt, [E] seinte Osith vunt chalengant K'en cest surfet est si suffrant.

Seinte Osith pas lunges n'endure,

1504 Bien li sovient de (sa) leidengure Suffrir ne vout pur nule rien, Ke vengée ne seit mut bien, $\mathbf{E}$ de celui nomeement

$1508 \mathrm{~K}^{\prime}$ (a) achesun fu premerement, Fonteine e surse e chief par non

De faire icele envasion, Kar ceo fu l'eveske Ricard,

1512 Venu i sunt cil de sa part Ke lur terres unt envasé E lur serganz unt hors buté, Miracle avint ici mut grant

$1516 \mathrm{Cum}$ les chanoines vunt plainant, L'ymage en fiertre unt envili Cum vus avez devant oï, Meïsme le hore fu notée

1520 De plus sages de la contrée, Un mal par tut le cors susprent Ricard l'eveske horriblement Sudain e laid de grant baillie

$1524 \mathrm{Ke}$ nomé est paralisie,

Ne puet ve[ei]r ne puet sentir,

Ne puet conustre hume pur veir,

$1528 \mathrm{Ne}$ il ne se peut del liu mover.

Saillient si hume, si sergant, Entre lur braz le vunt portant, Plurent ou mut horible cri,

${ }_{r^{\circ} \mathrm{b}}^{146}$ 'Sire:' funt il, 'pur Deu merci, 'Parlez a nus, cheles k'avez, 'Vus fustes sein oreinz asez.' Asez le vunt aresonant,

1536 Mes ne respont ne tant ne quant, Ne saveit en quel siecle $f u$, Tut l'unt pur mort iluec tenu, Tut issi jut deske (le) mardi,

1540 De ci ke vint al vendredi, Ne treit a sei ne main ne pié, Les seens sunt mut desconseillié, Par cele sale vunt pleinant,

1453 Cf. $\$ 53$, III, ii.

1469-76 'The seneschal came to Dakinton...and here considered whom he should send to Chich for he would drive those out who were put there by the canons; ecclesiastics he would put there who would give him accounts; the bishop had the intention of taking the canons from that seat.'

1474 MS. ou veskes eus.

1475 Cf. $\$ 20$.

1483 Corr. li chanoine unt....

1492 The MS. is not very plain here; I am uncertain whether to read tres[tut] estant or to correct tut en estant, since it is not clear whether 'on its feet' or 'immediately' is he real meaning.

1493 Cf. $\$ 17$.

1542 For mut cf, l, 1515.

1496 Cf. $\$ 53$ I, iii $\Delta$ (the $k e$ should be omitted) 
$1544 \mathrm{En}$ la chambre funt doel [mut] grant,

Kar al quer unt grant desconfort, $\mathrm{Ne}$ sevent s'il est vif ou mort.

A tant es(te) vus repairé sunt

$1548 \mathrm{Ke}$ seinte Osith deseisie unt;

La novele mut esturmie

De lur seigniur unt ja oïe.

$\mathrm{Li}$ seneschals vien(en)t au seigniur,

$1552 \mathrm{~K}$ 'il vei(en)t suspris de grant dolur, Williame ke ja est repairez

De la $u$ il fu enveiez.

'Sire, fet il, pur Deu merci,

1556 'Un poy ver moy entendez ci,

- Si de memoire rien avez,

' $U$ nul home entendre poez,

'E raison de clerc u de lay,

1560 'Dunc entendez ke vus diray.

'Beau sire cher, tres bien savez

' Vers Dampnedeu mespris avez.

-Vers seinte Osith nomeement

1564 ' $\mathrm{Ke}$ nus avum si folement

${ }_{\mathbf{v}^{\circ}}^{146}$ 'Ja desaisie de sa terre;

'Empris avum mut fole guerre ;

'Quei k'en apres seit fet de nus

1568 'La peine chiet primes sur vus.

- Kar par autre oy avum,

'E par nus mesnes le savum,

'A seinte Osith ne peut hom rien

1572. 'Mesfaire, ja ceo sachez bien,

' K'ele n'enprenge tel vengement

' $\mathrm{Ke}$ bien parra finablement,

' $\mathrm{E}$ ore l'avez pur verité,

1576 'Par vus me[i]smes esprové,

- Pur Deu tant cum vus poez,

'Sovaus de quer vus repentez,

' Kant est issi ke n'avez mye,

1580 'Force de lange ne de baillie,

- De quer criez a Deu merci

' $E$ a seinte Osith (tut) autresi ;

' $E$ voez li sa terre rendre

1584 ' $\mathrm{Si}$ ja vousist vers vus entendre;

' $\mathrm{E}$ Dampnedeu merci crier

- Pur vus de cest mal deslier.

- Pur Deu sire, car en pensez

1588 'Bien le veum mester avez,

- Pensez de ceo ke vus ay dit,

' Pur sa ducur Deus i ait.'

A ceo ke il ad parlé si,

$1592 \mathrm{Li}$ eveske puis s'esperi,
Ke aveit longement geu,

E sanz vigur e sanz verteu ;

Al seneschal k'ad si parlé

$1596 \mathrm{E}$ de son bien amonesté, De la main destre vereiement,

$\underset{v^{\circ} b}{146}$ Le dei ou tut l'anel li tent, Kar solunc ceo k'out en corage

1600 L'anel li tent e lui degage;

$\mathrm{E}$ cil li tret l'anel del dei

E si li dit: 'beau sire or vei

' $\mathrm{Ke}$ cest gage enveer volez,

1604 'A Deu vers ki mespris avez

'A seinte Osith nomeement,

'Pur li requere acordement,

' $E$ as seigniurs de son muster

1608 ' $\mathrm{Ke}$ desaise avum autre ier.

'Ore n'i ad en quant cest entent,

'Jeo m'en irray tut erranment.'

Li seneschal est ja muntez,

1612 (E) a seinte Osith tut dreit alez,

Veint a muster, fet apeller,

E les seigniurs trestuz mander,

Sur l'autel ad [tut] en apert

1616 En $1[$ e]i de gage l'ane] offert,

Crié merci a tut entur

De par l'eveske son seigniur

E lur terre tut quitement

$1620 \mathrm{E}$ as chanoines tantost rent

E (si) requert tuz les assemblez

Ke le maufait seit pardonez.

Meisme l'oure k'est venuz

1624 A seinte Osith receuz

Pur le mesfait fere adrecier

Dunt est venu tel encombrer,

Bien unt noté, bien entendu

$1628 \mathrm{E}$ de plusurs est retenu,

K'a l'eveske demeintenant

La u esteit [com] mort gisant

14? Este vos l'ole e la vue

$1632 \mathrm{E}$ la parole ja rendue,

Meismes en point e l'ure dreit

$\mathrm{Ke}$ li gage[s] offert esteit

Gariz esteit si come si,

1636 Mes nequedent pur veir vus di, Unk(es) puis sur piez n'estoit allant,

Ne pout estre pur rien estant

Mes hum li fist une chaere,

1640 Aturné[e] en tele manere,

Ke ileukes fist quant fut errant,

$1547 E_{s}$, est, este, estes seem all to be used, together with an inserted $l e$, according to the exigences of verse. Cf. 1. 905.

1551 The copyist appears to have taken li seneschals for a plural.

1576 Cf. 1.1623 and $\$ 64$.

1624 Something is wanting to complete the sense; $e$ fu might be inserted; the scansion of past participles with feminine $e$ is not fixed. Cf. \& 5 . It seems quite likely that the words should be tuz recreuz.

1631 I can only read the initial $E$ and following 8 and also what appears to be $v$. 
Tant cum en munde fu conversant.

Le merc tut dis bien li parut

$1644 \mathrm{Ke} \mathrm{jl}$ en tel mal issi recut,

Il puet ve[ei]r, il puet parler,

Mes sur [ses] piez nul pas aler

Dekes a sa fin verraiment,

$1648 \mathrm{Ne}$ li estuit nul autrement,

La chaere u fu portez,

E par la terre tant menez,

Esteit si cume fu furmée,

1652 A seint Pol de Londres portée.

Iluec remis est a tut dis

Bien le sevent cil del païs,

$\mathrm{E}$ cil ki iluec unt esté

$1656 \mathrm{Si}$ enquis unt, la verité.

Seigniurs, freres, pur ceo vus di,

$\mathrm{Ke}$ cest(e) example avez oï ;

Ne fet a Deu pas tel juer

1660 Cum a veisin ne cum a per.

Quant contre li pechent la gent,

Il est mut suffrant longement,

Mes tut seez de ceo seur,

${ }_{\mathbf{r}^{0} \mathrm{~b}}^{147}$ Ke quant il fiert il fiert dur.

Bien resavum la verité

Seinte Osith ad grant pousté

Ke Dampnedeu li ad doné,

1668 Asez reu et bien mustré
De sey venger e de ses enemis

Ke li mesfunt en son pais, E pousté read ensement,

1672 De mut valer a tute gent, Ke Ieu vondrunt e li servir, Mut bien purrat trestut merir Quanque hum frat pur li de bien,

1676 Ne puet estre perdeu pur rien. Ele est la sus en cel pais

$U$ Deu ad mis ses chers amis, Ne puet buche de hume parler,

1680 Oreile oir ne quer penser, Ne oil ve[ei]r en nule manere Cum la joie est iluec pleniere. A tuz ceus qui Deu amerunt,

$1684 \mathrm{E}$ de bon quer le servirunt, Seinte Osith nus seit en aye Tant cum sumes en ceste vie, Ke tant i puissum bien overer

$1688 \mathrm{E}$ de peché nos cors garder, Ke puissum au jur de la fin Vers Deu tenir le dreit chemin, E meindre ou ses chers amys

$1692 \mathrm{Ke}$ unt la joie de parais ;

[E] vers nus doint sa majesté

Ke maint uns Deus en trinité.

Amen.

1642 Corr. mund as in 1. 40.

1664 Corr. mut dur. Cf. 1. 1432.

1669 The sense demands the correction des enemis.

1674 Cf. \& 59 . 


\section{LANGUAGe and Versification.}

As few Anglo-Norman texts are accessible in good editions, it seems fitting to follow the method as far as possible of one of the best of these-Professor Stimming's Boeve de Haumtone-and to present this study of the language of the Life of St Osith in the same order; we can then refer to the pages in his edition where references are made to other texts and thus economise space. We shall refer to his edition as St. and to Suchier's Voyelles toniques as S. The comparison is made all through with Central French. The two portions of the poem which, as we have stated in the Introduction, appear to be of doubtful originality, will be referred to as (A) and (B) when they call for special notice.

\section{VoweLs.}

$\S 1$. French $a$.

This sound appears regularly as $a$ in conformity with Central French as is proved by the rimes in $a$, e.g. $33,165,173,467$ etc., also in (B) 221, 227, 348. Neither the scribe nor the author knew the form in ai (cf. Vie de saint Richard'). Other pure rimes in - $a$ are: -able 17; -acle 365 ; -age 71,375 ; -as 371,653 ; -ast 119, 563.

Before $l$, the development of Latin - $a$ tonic shows hesitation; thus side by side with the correct tel : ostel 1123 (cf. $§ 4)$ we find esperital 308 (cf. $§ 59$ ) but only in (B); the remaining rimes in -al [all in (B)] are correct-305, 307.

This hesitation of the ending <-alis is found often in Anglo-Norman and Norman, commonly in the West of France, while it is correct in S.W. French dialects. For Agn. Gaimar has ostel : criminel 545; mal : estal 3129. Adgar rimes mal: reial xvii 545 ; vassals : reials

1 Vie de saint Richard in Revue des Langues romanes, Oct. 1910. We shall refer to this life again simply as St Richard. 
xxxiv 93 ; mals : criminals xxviii 105 etc. In our poem both poet and scribe knew only the forms tel and quel. Before $r$ here as elsewhere in Agn. we find sometimes $e$, e.g. herneschant 913.

In the protonic syllable $a$ remains, at least in the orthography; nothing further can be said as there are no cases of rich or over rich rime. Thus we find laur 895; flael 1428 etc.; and after a palatal chaere 1639, 1649, and the learned chanoine 1263 etc., charité 1468.

Here as elsewhere in Agn. we have chescun.

The protonic syllable has disappeared in granter 1093, though it would seem to be required in the original text in line 723 .

Protonic initial $a$ is sometimes replaced by a different prefix en as entendirent for atendirent 803; enchesoun 304 for achoison or achoise, ensemblé 428.

[St., pp. 172-3.]

\section{§2. French $\tilde{a}$.}

The Agn. custom of writing -an as -aun is rare in our poem; we find only auntes $206(\mathrm{~B})$; the rimes in -ant are all pure, cf. 61, 185, 473, 535,541 etc. and $317-9$ in (B), also those in -ance 157 and in -andre 41 ; orient and talent which hesitate in Agn. and Norman are here found only in rime with -en, cf. $\S 6$. Here, as commonly in Agn., we have uncore, a form which may be due to analogy with unc, onc $<$ unquam. In the protonic, the scribe at any rate uses en, e.g. ensenglanté $817,823$.

[St., p. 173 ; S. $§ 39$.

\section{$\S$ 3. French open $e[\epsilon]$.}

After $e$ and before $u<l$ a glide $a$ has developed; thus beau passim; this sound may then rime with -aus<al $+s$, cf. beaus : chivaus 25 (cf. $\mathrm{S}$. $\$ 58 \mathrm{a}, 59 \mathrm{~b})$. The rimes in $-e$ are mainly pure, cf. chancel : bel 1203; mantel : pasturel 331 (B); novele : ancele 433 (cf. S. $§ 15 \mathrm{~b}$ ): apele 635 ; anceles : puceles 49 ; pucele : bele 169 ; apres : confes 965 : ades; pert: sert 5 ; apert : cert 189 : offert 1615 ; tempeste : moleste 865 ; those in -ere are furnished only by terre, guerre, and quere or their derivatives, cf. 83, 380, 1075, 1085, 1163, 1565.

There is some hesitation with est, it rimes correctly with prest 571 but also with icest 543,729 ; a similar rime may be found in the Tristan of Béroul 2049 and in the Bestiaire of Philippe de Thaon 881 est : met < mittit, and with this may be compared the rime ere (< erat) : pere in Sainte Marie l'Égyptienne $511^{1}$ and Énéas 2159, 3927.

The words mariners : juvenels 883 have been corrected in a footnote to the text.

[St., p. 174.]

1 Our edition of this interesting life is to appear shortly. 
§4. French close $e$ [e].

Central Old French possessed two varieties of close $e$ which may have differed by length only:

A. Close short $e<$ Latin checked $\bar{e}$ and $\grave{\imath}$ exeept before nasal consonant. There is no satisfactory rime on this sound; richesse: tristesse 15 proves nothing as both represent -itia ${ }^{1}$ but the former should give richise; the other -chiiet : met 601 is hardly satisfactory; here chiiet of the MS. or perhaps chuet, since the right to left strokes which are usually found over $i$ are here absent, would seem to be for calet, the word does not appear elsewhere in the poem but it is found in Agn. as cheut and chiut (Central French chieut) in the Quatre Livres des Rois and in Samson de Nantuil who rimes chiut : suit (cf. Suchier $\S 32 \mathrm{a}$ ). If chuet is the correct reading of the MS., it seems likely that it is an alteration of the scribe for cheut; this ehange of spelling should indicate a pronunciation $\ddot{o}$ which varied between $\alpha$ and $\phi$ aceording to the eonsonant which followed; the presenoe of the labial nasal in met may have produced an effect of rounding (the $\breve{e}$ is very rare in rime ${ }^{2}$ ) and have caused the poet to consider the rime good enough. Another solution seems possible and this is that the poet wrote the form chiet; $i e$ standing for ieu, a reduction to be found in several Norman and Agn. poets, cf. fieu > fié, e.g. fiez : veziëz, Marie de France, Eliduc 63; this would then give a rime $i e ́: \breve{~}$; similar rimes are found e.g. clers (< clĕrieos) : fers (< fěros) St Gilles 2241 ; clerz : querz in Frère Angier, Grégoire $3 \mathrm{r}^{\mathrm{O}} \mathrm{a}$.

B. $e$ long < Latin $a$.

The instances are extremely common and words with this aceented vowel form a large pereentage of the rime words. The rimes are pure, viz.:

$$
\begin{aligned}
& \text { é } 21,93,95,129,143 \text { etc., also in (B) } 205,327 \text { etc. } \\
& \text { ée } 69,127,171 . \\
& \text { ef nef }: \text { tref } 945 \text {, also (B) } 276 . \\
& \text { el tel : ostel } 1123 \text {, ef. } § 1 .
\end{aligned}
$$

1 What should be the outcome of -itia is a much discussed difficulty; we have richise (Poème moral); richeise (Adgar $\mathrm{xxx} \mathrm{29);} \mathrm{St} \mathrm{Gilles} \mathrm{has} \mathrm{richeise} \mathrm{and} \mathrm{richaise} \mathrm{and} \mathrm{richesce}$ $356,277,549$ but only richeise (:peise) in rime; Bestiaire lias justise : mise 67 and richeise: depreise 1409. We may suppose richesce to be a younger form.

2 For the scarcity of this rime cf. S. $\$ 16$ e and Förster in Zeitschrift xxvmr, 508; the tirades of any assonanced chanson de geste will if long be found on examination to consist largely of diminutives as final words; in rimed poems, e hardly furnished $\cdot 1$ per eent. of the rimes, thus met occurs in rime only thrise times in Wace, cf. Pohl in Rom. Forsch. II, p. 544; twice in Benoit, ef. Stock in Rom. S'tud. 111, 450; twice in Énéas 3721, 8981 (metent : getent 3833); once in Adgar 29. 46 once in Ste Marie 1083, and not at all in St Gilles or the Adamspiel, while in the Bestiaire we find met : bec, the vowel of which is probably open, ef. $\$ 3$. 
er infinitives of the 1st conjugation are very numerous, ef. $\S 17$ (for targer : saver 304 ef. $\$ 15$ ).

ere rime furnished only by pere : mere $23,133$.

ez 2nd person pl. of verbs and a few other words as delez : prez 323 and the endings -atus, -atos.

Different spellings are used to denote this sound, viz.:

(i) $e$ as above and also in $e l<$ alum for aliud 1301, heent 796 , sevent 185 , set 96,544 .

(ii) ee as neez 32, cf. also $v v$. 369-70.

(iii) ei as asenseie (: veie, ef. $§ 15) 1181$.

(iv) $i$. as in the form remis for remes < remansum; this past participle is found in certain Agn. and Norman works, cf. Suchier $\S 17 \mathrm{~d}$.

[St., pp. 175-6.]

\section{§5. French atonic $e$.}

A. Before the accented syllable.

Side by side with the correct and usual form $e$ as in chevache 601, chemin 273, cheminal 307, acheminé 270, enchesoun 304, poseer 1453, repelle 378 etc. we have other spellings:

(i) a asaer 937 , manascer 703.

(ii) $i$ chival 26, 600,605; cristiene 795 ; diable 1313; ordinez 417, 1251 ; primere 157 .

(iii) o (u) sucur 125 ; solunc 1599; pour 630 ; suveus. 344 sovaus 1578; pousté 1666.

(iv) In the $3 \mathrm{rd} \mathrm{pl}$. pres. indic. -ent appears frequently as -unt, e.g. atendunt 28 etc.

Very early (certainly since the fourth decade of the twelfth century) Agn. words are found in which atonic $e$ is either omitted or not counted in scansion. The earliest to show this reduction seem to be nouns in -ure as the porture 380 , enveisure 992 of our text and a compound like bonurté and perhaps bonuré 127 ; next in order come past participles in - $\ddot{e} u$ as $j \ddot{e} u$, vëu, cf. 1153; and granté, cf. 1214, then the corresponding preterites as our decut 1400, jut (: apercut) 1139; about the same time is to be placed the mutation of the feminine $e$ of adverbs: drein 871; memes 246, 1570 (but cf. 1576); seurement (cf. 88), veraiement.

B. After the accented syllable.

It is a sign of late thirteenth century Anglo-Norman to find the final $e$ wanting ; except for $u n^{\prime} 869$ and $e l^{\prime} 1573$, this occurs only in (B) disai 201, merveil 266 (: conseil), chai (:obli) 281, but c̣. $§ 55$ II, chausé 335. 
It is doubtful whether the rime verité : voe 527 is a case of disappearance of final $e$; al though only voée seems to be known in the sense of vow, it is possible that a masculine form may also have existed; it should be noticed that the adjective is in the masculine. In the Haveloc we find avowé as a masculine (:adubbé) 927.

We may now enquire into the syllabic value of this $e$ and 'we shall consider (B) apart.

I. In the following cases $e$ appears in the orthography and is syllabic.

(a) Nouns: deuble 1309,1313 ; ordenez 660 ; reïne 134, 688; seüs 562 ; in line 697 the initial word mes should be omitted.

( $\beta)$ Pronoun : ele 1388.

( $\gamma$ ) Verbs: (i) past participles: creu 1332 ; leue 67 ; seu 68, 1042 ; peu 115; veu 444, 561, 927, 1064, 1306, 1402 and 309 (B).

(ii) other parts of the verb: deusent 1453; deseelez 1409; poseer 1453; veum 21; veeit 168; veeir 348 (B).

$(\delta)$ Adjectives and adverbs: derein 1097 ; verai 59 ; nomeement 1300, 1605.

( $\epsilon)$ The $e$ is also counted after a vowel with following consonant: contrée 186 ; guarrie 1284 ; portée 1217 ; venue 1212 ; voie 1170.

$(\zeta)$ The word sire which in Agn. often counts for one syllable only (cf. St Richard $§ 77$ ) is here dissyllabic, e.g. 1142, 1161, 1587.

II. In the following cases $e$ appears but is not counted.

(a) Nouns: deable 696, 1303 (cf. I (a) above); havene 877 etc.; in abeïes 784 the line appears to have been altered by the scribe who has inserted la unnecessarily.

( $\beta)$ Pronouns: une 536 ; ele 1246, 1301, 1388, 1572; cele 1177, 1231 etc.

(y) Verbs: purveu 1153, especially with the combination mute +liquid: enbeverez 493 ; troverez 1173; severez 1364; deliverat 1408; avera passim; since teoldé 874 is not found elsewhere, it is difficult to decide, but as tialz (see footnote to 1.874 ) is dissyllabic the verb should be also, the line could be emended by omission of bien.

$(\delta)$ Adjectives and adverbs: veraie 1286 ; vereiement 1597 ; seurement 88 ; meimes 493, 575; cume 1398.

(є) Preposition : dekes $=$ dek' 1018 and elsewhere.

An $e$ may be inserted contrary to etymology and will then not be counted, e.g. creient 5 ; empouerist 692 ; perdeu 169, 1676; seurveillie 1104.

In the section we call (B) the accented vowel is generally silent 
in any position, thus: age 264 for eage; ewe 273 ; sust 253 ; seumes 338 ; peust 360 ; deust 359 ; preist 228 ; veistes 329 ; veimes 334 ; venir 261 ; appela 241 ; amedouz (fem.) 341 influenced by ansdous; une 290.

\section{§6. French $\tilde{e}$.}

[St., pp. 176-84.]

The nasal is invariably represented by en and the rimes are all pure, there is no confusion of en and an (cf. $\$ 2)$. Rimes in -ent (41 in all) are pure as e.g. prent : follement 9 ; entent : sovent 101 etc.; even talent and orient rime with vent 906, premerement 1341 and with rent 837 ; the same is true for (B). The purity of the rimes is carried through even before a heavy consonant group, e.g. prendre: atendre 657 ; entre : ventre 611.

As in Central French we have volentiers 119, 250. The change of the suffix $a$ to en as in enchesoun 304 (compared with the correct form achesun 1. 1508), common in thirteenth century Agn., is attributable to the scribe. Cf. $\$ 1$.

[St., pp. 184-5.]

§. French $i$.

This vowel appears regularly as $i$ : belif 947 ; dis 1653 etc.; the spelling $y$, frequent in the latter part of the thirteenth century (cf. Stürzinger, Orthographia gallica, rule 17), is little used in our poem; we find however ly 549; say 1162 (but sai 1164); noyse 536; ymage 1492 ; Ynguar 770.

The rimes in $-i$ are pure, consisting chiefly of past participles of the second conjugation, e.g. 1l. 57, 149, or of ci, si, merci etc., e.g. 187, $351,353,471$. Rimes in $-i e$ are pure also, e.g. ll. 75, 123 etc. The same is true of the following rimes: -ir (mainly infinitives) 75,413 , 453, 457, 497 etc.; -ire 445, 455 etc.; -ist as fist : prist 117 ; -it 463, 495,1175 ; -is 393, 399 etc.; -ise 361, 693, 1245; -iz 233. This $i$ comes regularly from -iei as $m i 278$ and may be in rime with $i<$ Latin $i$; cf. 1. 471. In the protonic $i$ appears also as deliat 1407; otria 249 ; septimus is represented by sime 1240 by analogy with dime, disme and the ending -ime.

In Agn. the spellings $e i$ and $i e$ are found sporadically, e.g. preisa 165,168 ; vierent 1200 .

[St., pp. 185-7.]

§. French $\tilde{\imath}$.

This sound is represented by - in as fin:chemin 27, 1169; enclin 637 ; the rimes in -in are pure as too are those in -ine 697 ; -int 215, 425,485 . There is only one case of -ein for -in, viz. veint 1613. For creient see $\$ 18$.

[St., p. 188.] 
$\S 9$. French open $o[0]$.

A. $\quad$ < popular Latin $a u$.

This sound is almost exclusively represented in Agn. by 0 and it would appear to keep its open quality intact; there is only one rime in our poem--chose : pose 533; other words are: or, unkore, noe 591; estorer 741 ; loer 56; usım 653; oster 1115; otreia 222; and the various parts of the verb oïr. The reading out 433 should doubtless be ot as in line 95 while the outcome of apud figures often as ou 238, 628, ef. $§ 24$. For this ou cf. Miss Pope's Etude sur la langue de Frère Angier; St., p. 189 and S. $§ 13$ c.

B. $0<$ Latin checked and atonic $\breve{~}$.

This sound is also generally represented by o; e.g. col 281; cors 347 ; fors 348 ; fort 610 ; folie 1425 ; novele 1549 ; orer 886 ; volage 239. Since the rimes in -ose are so rare in Agn. we may quote the following:

Adgar chose:glose 36.42 ; : rose 9.143.

Simund de Freine chose: pose St G. 855.

Vie de Ste Catherine chose : ose 1299 : pose 2063.

Chardry repose:Theodose S.D. 831 ; rose : disclose S.D. 623.

Vie de Ste Marie l'Égyptienne chose:rose 467.

Vie de St Richard chose: parclose 27.

Disputeison de l'ame et du corps chose : rose iv 3,6 .

Suchier $(\$ 13$, a 4 ) admits as providing open $o$ in French a number of borrowed words where $\zeta$ or $\bar{o}$ are in open syllables.

[St., p. 189.]

$\S 10$. French close $o[0]$.

French close $o$ has doubtless the sound [u], i.e. ou in Agn. and the MSS. use this symbol very extensively. In our poem the words riming on this sound have generally $u$, e.g. creatur: seigniur 9 ; honur:amur 39 ; tur :jur 1103, 1149 etc.; other than in rime the stressed syllable is always represented by $u$, curuce 684 ; dutuse 109 ; joiuse 230 ; mustre 1 ; tut is also found as tuit, e.g. 48. In the syllable under secondary stress both $o$ and $u$ are found and occasionally ou: sovent 100 ; doter 35 ; mustrer 343 ; purrum 75 ; oure, houre 865 but hore 569 ; nus, vus, cum, but, mut, dunc always appear with $u$ and spellings with $u$ form about eighty per cent. of the whole; the spellings ou and $u i$ seem rare: voua 124 ; tuit 381 ; ou $<0+$ vocalised $l$ remains.

[St., pp. 190-91.]

§.11. French $\tilde{o}$.

Although $u$ is so common for $o$, $u$ is less frequent than on. In the rime words on occurs with only two exceptions-ll. 675, 773; the 
first plural ending is always -um; conseil and compounds of cum- are regularly com-; doner is always so spelt; son is much commoner than sun, but curiously enough while -ons is always so spelt, unt is preferred without exception, the same is true for -unde 587; we find both long and lung, longement and lungement; the ending -ion is constant. The spelling -oun is rare.

[St., pp. 191-2.]

$\S 12$. French $u$ and $\tilde{u}$.

The sound is represented by two spellings, $u$ and $u i$, the second is rare; there seems to be only one example in our poem: estuit for estut 1648. The rimes are pure, e.g. those in (i) $u 81,115,505,523$ and in (B) $195,309,311$; (ii) ue 67, 443; (iii) urne 875 ; (iv) ure 1, 355, 701,991 ; (v) us 107, 561, 1223; (vi) ut 181, 1137 ; with one exception aventure : oure 547. As is common in Agn. de usquam appears as deke, dekes.

The nasal $u$ is rare, it occurs once only in rime and offers no varieties in orthography.

[St., p. 193.]

$\S 13$. French ai.

The sound ai began early to develop into $e$; among the first words to show this are ewe 273 , lermes 1499 or where ai is followed by a heavy consonant group, e.g. mestre 209, nestre 193; later the change is found in any enclosed position. The change is more complete in Agn. than elsewhere and occurs in any position; the sound varies between close and open $e$. When ai was final it appears to have retained its diphthongal pronunciation throughout the thirteenth century. The change is explained as taking place through ei and all three spellings, $a i, e i, e$, are to be found. Examples of these are: contraite 1067 ; fait 138 ; feit 478 ; treit 168 ; fet 4 ; mes 238 ; trere 1196 . This last spelling is perhaps commoner than either of the others. The reduction of $a i$ to $a$ seems only to occur in unstressed syllables, e.g. paens 763. The spelling ai occurs also for ei, e.g. disaie: tuchaie 201 (B). The rimes in $a i$ are strikingly pure: (i) ai diray: lay 1559; otherwise this rime is furnished only by the ending of the first person of the preterite or future as in 1l. 199, 315,989; (ii) ait mesfait : plait 111 ; ait : vait 553 ; cf. also 1l. $669,933,967$ against an incorrect rime vait: dreit 899; (iii) aire pure for faire : traire 1355 and here : trere 1495 while for feire (< fēria) : trere 1195 we may assume that $e i>a i$ before $a i>e$ and this is supported by the modern spelling fair; the single rime in -aite is pure also, despite the spelling, contraite: desheite 1159.

[St., pp. 193-6.] 


\section{$\S 14$. French aĩ.}

This sound is generally represented by ein, e.g. meins 785 ; pleint 1242 ; seinte no other spelling; seinteté 236 etc. The rimes are with one exception pure, e.g. mains : noneins 785 (the spelling of the MS. is retained); main : à plein 781, 925, 943 (plein<plano, cf. G. Paris, St Gilles ${ }^{1}$ xxvii); drein : morlhein 871 but main : sein (< sinus) 667. The rimes in aine are, however, confused, funtaine : meine 799 ; peine : semeine 1295 ; that in -aint is pure, remaint : plaint 1125 . The result of an enquiry I have made into these rimes in Agn. texts of the twelfth century ${ }^{2}$ is as follows:

1. Philippe de Thaun:

(a) Comput. No examples of confusion.

(b) Bestiaire. Onc doubtful case, cf. Walberg, Introduction, p. xlviii.

2. Reimpredigt. No faulty rimes.

3. Gaimar. One faulty rime in ain, viz. plain : serein 767. Vising in his Etude sur le dialecte anglo-normand $d u$ xi $\ddot{\imath}^{\theta}$ siècle, p. 84 quotes as instances of confusion grifaine : quinzaine 3007 and plein: main 4033, but grifaine of the first is regularly found in rime with words in -aine and aigne, and plein of the second example might conceivably be for vlain, such is the reading of one at least of the MSS.

4. Adgar, Miracles. Fiftcen cases of confusion.

5. G. de Berneville, St Gilles. No confusion.

6. Sainte Marie l'Égyptienne. Only one clear case of confusion, quarantaine : ceine 747.

7. Le Donnei des Amants (Romania $\mathrm{xxv}$ p. 497 seq.). sein is found twice in rime with main 787,801 and once with vilein 831.

8. Chardry. 'Two cases of confusion.

Our poem is therefore in this particular not more advanced than several poems that are older.

[St., p. 196.]

§15. French $e i$ (later oi).

This sound occurs in Agn. correctly only as ei, e.g. dei 1598; eire 258; feire 1195 (cf. $§ 13$ ); feiz 346; neir 331 ; also $e i<e+n$ and $l$ mouillees as in deignast 343 ; esmerveilliee 302 . The following spellings are also found: (i) $e$ in aparer 617 ; aver 98 ; mescrere 1319 ; (ii) $i$ in

1 So also in Wace: E prent quanque il treue a plein, Rou 1698.

2 I have, of course, noted special works on the subject as Vising, o.c. (1882) and Rolfs, Adgarlegenden in Vol. I (1883) of the Romanische Forschungen, though it will be noticed that I do not alweys arrive at similar results; this is due to the fact that better editious are now available. 
damisele 274; deviez 1403; (iii) oi in moi 244; damoisele 315, 329. [Note these are only found in (B).]

The sound is probably still slightly diphthongal; it is only found in rime with itself as is shown by the following lines: (i) -ei in fey: ley 141, 423; rey: mey 451; (ii) -eis in reis: Engleis 131; (iii) -eil in veil: peil 689; (iv) -eille in merveille:veille 1193; (v) -eir in veir :poeir 509; (vi) -eit in esteit : aveit 517; espleit : veneit 559 : dreit 784 (cf. $\S 54 \mathrm{iv})$.

Our poem contains four instances in which veie < via is in rime with feminine past participles <-ata, viz. alée 811 , asenseie 1185, portée 1207, entrée 1333. This is not a proof that $e i$ has become $e$ but that $e$ followed by a feminine $e$ has a tendency to diphthongise. There are instances of this in Frère Angier, e.g. veies: remuées (see Miss Pope's Etude sur la langue de Frère Angier, p. 11), and also in Chardry, monée $<$ moneta: dunée and aportée [Sept Dormanz 1205, 1291]. For Chardry the rime doubtless indicates that $e i$ has become $\dot{e}$ but our text presents another instance, viz. the rime levée : desree 537; here desree stands for desreie and is therefore another instance of -eie :ée. A similar rime is to be found in Aiol, a late twelfth century text with N.E. dialectic characteristics, desree : trainée, while like rimes are common in W. and S.W. dialects. It is one of the points of interest of Anglo-French to find it in agreement with continental dialects. Professor Stimming quotes (p. 175) a number of instances in which ée <-áta appears as eie, e.g. espeie, valeie. We have seen $§ 4 \mathrm{~B}$ above that $e i$ is a recognised spelling for $e$, it is frequent in the Corpus Christi (Oxford) MS. of Sainte Marie l'Egyptienne where this sound occurs in the last word of a line. There is a tendency also in Agn. to insert $i$ between the antitonic and the tonic vowel, e.g. ayé (<ætatem), veyer (< vetare) in Boeve de Haumtone; loiée (<laudata) in Destruction de Rome 32, an Agn. MS. of possibly some other dialect (cf. Romania II p. 5).

The forms aver, poer, saver etc. found frequently in rime in Agn. with infinitives of the first conjugation are probably cases of analogical formation (cf. P. Meyer in Bozon, Contes, p. lxii). Instances of this would seem to occur about the end of the twelfth century; the earliest examples I have met are in Le Donnei des Amants (dated by G. Paris at the end of the twelfth century); aver: aler 980 , : doloser 1144; the cases quoted by Rolfs (l.c. p. 209) from Adgar are only in poemsGregory and The Abbess delivered by the Virgin - of doubtful authenticity; in the remaining poems Adgar's rimes are pure. Our poem has one inștance but only in (B), the doubly incorrect targer (for targier): saver 
303 and with this nay be compared esmaer:veer (< videre) in the Legend of Gregory 1750 just mentioned.

$\S 16$. French $e \tilde{\imath}$.

[St., pp. 197-200 and 237-8.]

The sound is represented by -ein as sein 668; ameine 401; peine 402 ; for the rimes cf. $§ 14$.

$\S 17$. French ie.

The sound is represented by $i e$ and by $e$ : (i) ariere 542 ; chiet 1361 ; ciel 8,503 ; fiert 1664 ; siecle 36 (this word is generally so spelt in Agn.); (ii) aider 121; cel 377 (this spelling is rare compared with ciel which is found in texts which have elsewhere regularly reduced ie to $e$ ); meuz 89 ; mester 232 etc.; neces 154 etc.

The reduction of iée to $i e$ represented by the orthography of 11 . $1221-2$ is not supported by a rime with $i e<i+a$ etc. The rimes in $i e$ are singularly purc. In the passages that may be ascribed to the original author no cases of mixed rimes, ié: $e$, are to be found; posé : cungé 1497 ; loer:cher 1497 are in the Episode we call (A). In the main body of the poem there are 145 rimes in é (é,ée, ées, ef, el, er, ere, $e z$ ) and 49 in ié (ié, iée, iées, ier, iez). In the passage we call (B) there are 18 pure rimes in $e ́, 3$ in ié and 8 mixed, viz. ll. 207, 209, 229, 231, $301,303,329,367$. Suchier has compiled a list $(\$ 29)$ of words which are found, now in rime with $i \dot{e}$ and now with $\dot{e}$; of these our text contains-regné : ensemblé 428 : volunté 745 ; malvesté : enticé 1323; deviez (<de + vita + are) : aler 1403; crier : deslier 1585; oublié : esté 629. These may be left out of our calculations. The question of mixed rimes in Agn. is a very interesting one. In the earliest poets such infractions are rare but they steadily increase till a pure rime becomes a mere matter of chance. I have drawn up some statistics of the proportion of these mixed rimes in Agn. writers : thus we find

$\begin{array}{lcccc}\text { Author or work } & \text { No. of lines } & \text { Pure } & \text { Mixed } & \begin{array}{c}\text { Proportion of } \\ \text { pure to mired }\end{array} \\ \text { Gaimar } & 6534 & 586 & 9 & 65 \cdot 1 \text { to } 1 \\ \text { Adgarl } & 6870 & 612 & 10 & 64.2 \text { to } 1 \\ \text { St Gilles } & 3794 & 489 & 12 & 40.9 \text { to } 1 \\ \text { Haveloc } & 1106 & 129 & 6 & 21.5 \text { to } 1 \\ \text { Sainte Marie l'Egyptienne } 1534 & 171 & 13 & 13.3 \text { to } 1 \\ \text { Sainte Osith } & \text { (A) } 278 & 36 & 2 & 18 \text { to I } \\ & \text { (B) about } 204 & 21 & 8 & 2.62 \text { to } 1\end{array}$

1 Adgar has the peculiarity of finishing go many of his legends with the words...Dame chiere: preiere that it is necessary to make some allowance; we have deducted therefore 40 from the number of pure rimes and from the number of total lines; the two poems named in $\$ 15$ have not been considered for this list; they contain many cases of mixed rimes. 
I am not inclined to lay much stress on the proportion of pure to mixed rimes as a factor for determining the date of Anglo-French poems, but it is interesting to see how the proportion of mixed rimes corresponds roughly to the date of production. The matter of the dialect is too complicated for any one phenomenon to be decisive; it is necessary, as G. Paris stated (St Gilles, Introd. p. xxv) as long ago as 1881, to know something of the writer's upbringing. There is also the larger question: how many poets whose works show anglicisms are really English born or only immigrants? It seems fairly certain that Frère Angier came from a S.W. French province and that his language had become contaminated by residence in England $^{2}$; the same, I believe, is true for the author of the Destruction de Rome (published in Romania I, pp. 1 seq.) who would seem to be a native of one of the North or North-Eastern provinces and by a lengthy stay in England could be content to allow such words as celer (1. 269) to find a place in a tirade of ié assonances or chargié (942), asiegier (1461), Oliviers (1501) etc. into others that contain only $e$ assonances.

[St., pp. 201-2.]

\section{$\S 18$. French $i \tilde{e}$.}

The sound is represented by $i e n$ as in bien 5, 1270 ; criendre 106; it is reduced to -en only after $i$ as terrïen 60 ; crestïens 768,795 . The spelling creient (ll. 3, 5) may also be noted here (cf. $§ 5$, ii $\epsilon$ ). The rimes in -ien are all pure; bien : rien 167, 389; mien : rien 437. Other Agn. authors are less careful; thus we have in Gaimar ancienz:anz 1785 ; in Adgar crestiens : sens 5.101 : tens 6.223 etc. Mention apart must be made of the word nient; it is monosyllabic in lines 47 , 707,1363 and dissyllabic in $576,663,845$. It is generally found in rime with words in -ent as entent 663 and with adverbs in -ment, e.g. 11. 576, 846, 1392. This phenomenon is noticed in the earliest Agn. texts: thus in the Bestiaire (nient is not in rime in the Comput) we find that it is both mono- and dissyllabic and that it rimes in the same way as here (cf. Walberg's edition p. li). Gaimar has nient: descent 4019; Adgar nient: gent 31.33 : maintient 30.175 etc.; St Gilles neent (and nent 489): pulent 222 : verraiement 1100.

[St., p. 203.]

\section{$\S 19$. French ieu.}

This sound occurs in a very limited number of words and the spellings $e u$ and $i u$ are the most frequent; our text offers: Nominative

1 See the work quoted in $\$ 15$. Thi; would seem to be true for Marie de France, whose later works show anglicisms such as the earlier ones do not contain; see G. Paris' review of L'Espurgatoire in Romania xxiv p. 290 seq. 
case, Deus 117, Dieu 434, Deu 1423; Acc., Deu 31, and Dampnedeu very frequent for both cases. The form used for purposes of the rime is commonly in Agn. ${ }^{1}$ and many French dialects Dé, e.g. : overé 1206. We find also in our poem geu 992 ; giu 478; liu 192, 924.

[St., p. 204.]

$§ 20$. French $\partial i[$ ij].

Very few words with this diphthong exist; it is represented here by oi and oy, e.g. oi (< habui) 318 ; soi (< sapui) 320 ; poy (< paucum); this last is the common form in Agn. In the dialect of our text we find that $\breve{\gamma}+l$ mouillée gives oil and we have doil 1116; oilz 502 ; and voil : soil 1475. The word soil does not appear in Godefroy in the Latin sense of seat which it appears to have in line 1476 but only in the sense of souiller. We have also the rime estoire : memoire 97 ; this is a stock rime in Old French, in fact only the learned words can be used for the purpose. Instances of this are-Bestiaire, gloire : victoire 205. Gaimar uses as rime words: estoire, gloire, memoire, tempoire, victoire, see Il. 3239, 3311, 5711. Adgar uses estoire and memoire. Most Agn. scribes write -orie but this is not the case in our MS.

§21. French ó $[$ oi $]$.

[St., p. 204.]

The diphthong is somewhat rare, it occurs in Agn. as both oi and $u i$, e.g. voiz 383 ; anguisse 517 ; $u$ i appears to be preferred in the protonic as anguisuse 389 and fuison 1464.

[St., p. 205.]

$\S 22$. French oi. This too is rare, it appears as oin and uin, e.g. doint 349 etc., luintain 187, puignant 549. It does not appear in rime.

[St., p. 205.]

$\S 23 . \quad F r e n c h \grave{o u}[\mathrm{ou}]$.

This sound only occurs in our text in verb forms: out $<$ habuit 1441 ; plout<placuit 1442. This favourite rime in Agn. only occurs here twice and with the foregoing words. For the ending -out in imperfects of the first conjugation, see $\S 54$ iv.

[St., p. 205.]

1 The treatment $\sqrt{f} e u$ and $i e u$ in rimes needs special iuvestigation: for Deu it is interestiug to note that certain texts only rime with a limited group of words e.g. Gaimar Deu : feu (germ. fehu) 4321, : leu 1410; Adgar, Deu: Jueu 5. 13 : Andreu 19. 51 and this is also true for Braudan; whereas Philippe de Thuon, Fantosme, Sanson de Nantuil, St Gilles, Ste Marie and also Wace rime Deu with any word in $\ell<a$. But for the rarely occurring Nominative Des or Dens the rimes available seem to be few (cf. $\$ 32$ ). Thus in the Bestiaire Des : csperitels 195; Gaimar : remes 3601. The rime of $e(u) s$ so frequently with remes may be more than a mere coincidence and may indicate a labialised pronunciation of $e$ (cf. $\S 4$ ) for we have the rimes remes : tex in Benoit, Troie 19495 : Idomenes ibid. 28921, : ostels, Wace, Kou III, 4869, 4888 aud frequently in Chrestien do Troyes, : Kes, Yruin 2179; : ostels, Erec 5697; : Gres, Cliges 3623, 4212. 
$\S 24$. French óu [ou].

Few words only contain this sound. Here we have dous and in (B) deuz 346 and amedeuz 341. The outcome of $u b i$ is $u 188$ and ou 367; ou is often found for apud (cf. $\S 9$ ) and in its place our MS. has eu (ll. 390, 392).

[St., p. 206.]

$\S 25$. French $u e$.

This sound is represented in a variety of ways :

(i) ue: estuet 392 ; iluec 617,1108 ; puet 190 ; suer 1095, 1217, 1220, 1229; quer 305.

(ii) u: estut 35 ; iluc 1008 ; vus (for vues or veus < vŏles) 1141.

(iii) oe: poeple 488 ; iloec 298.

(iv) 0 : ilokes 875 ; volent 664 ; bor (also bour 31 ) 1424.

(v) eu: leus 323 ; peut 121 ; seur 242.

(vi) As the outcome of aviolus our text has aels instead of aieus. We have considered voil and soil in $\S 20$; the form seur is found in (B) in rime with amur 221 and with ducur 241, cf. $\$ 43$ iv.

[St., pp. 206-7.]

§ 26. French uथ̃.

The spelling uen seems almost unknown in Agn. Peter of Peckham (St Richard) has suens side by side with seens, which form occurs in our text 1542; the pronoun from homo is l'em 94, otherwise homo and bonum only give hom and bon.

[St., p. 209.]

$\S 27$. French $u i$.

This sound is found in our poem represented by $u i$ and $u$. Most of the rimes are pure, e.g. bruit: tuit 999 ; ennui:sui 643 ; fuive : destruire 767. In Norman and Anglo-Norman $u i$ is often reduced to $u$ when $s$ follows and in Agn. in conjunction with other letters. This $u$ may then rime with original $u$, e.g. jut : duit 297,1121 ; us < uis : us $<$ usum 1121. There are no cases in our poem of rimes of $u i$ with $i$ such as are to be found elsewhere, e.g. Gaimar qui (< cogito) : midi 1645; transit: quit 5137; Adgar qui:ami 28. 143. Nor are there instances of $u i$ : oi as destruiz : croiz, Adgar 30. 31 .

[St., p. 209.]

\section{Consonants.}

$\S 28$. Liquids.

There is no rime in our poem to prove that $l$ is vocalised; no pout: volt as e.g. St Gilles 3619 ; on the other hand there is nothing to prove the contrary. The same is true curiously enough for Adgar, Haveloc, 
and Sainte Marie l'Égyptienne, while the proof of vocalisation for Gaimar rests on the evidence of a single rime enchascout : volt 2003.

It is possible that the rime of $11.601-2$ discussed in $\$ 4$ is a case of the vocalisation of $l$. This absence of any decisive rime must be a matter of pure chance. Here as in many Agn. works $l$ often remains in the orthography as in al 281, 518 and del 691 ; these forms are normal ; our poem has also aels 205 (cf. $§ 25$ ); malgré 96 ; malvesté $1324 ; l$ is inserted in alme 691, and by the scribe reduced to $u$ in espaumes 891 ; paume 892 ; voudrai 642 . It has disappeared after $u$ in ducur 178. Sporadically in Agn. we find $a+u<l$ reduced to $a$; instances in our text are chevache 601; acune 858; sav(er)ast 119'. This is not a mere scribe's error as is proved by the English save. Other examples I have met are savaciun in St Richard 6 and maveis in Modwenna $17 \mathrm{~d}$.

The $l$ mouillee is represented by $-i l$ when final, e.g. conseil : merveil 265 and intervocalic by -illi- as ailliurs 6 ; fillie 130 etc., or by -ili- as perilié 972. There is no rime in our poem of $l$ with $l$ mouillée as is often the case in Agn., e.g. soleil : feeil in Bestiaire 2233; gopil : il in Gaimar 281 etc. fedeil : conseil in Adgar 28.57; Marsile (= Marseille): vile in St Gilles 1035.

The metathesis of $r$ seems only to occur in pernum 100, pernent 773, pernunt 1035. Anglo-Norman texts show great confusion with this consonant and spell the same word, now with $r$ and now with $r r$, as e.g. orez 108, orrez 113. A number of cases of double letters are treated together in $\S 41$.

The pronunciation of $r$ in Agn. is very weak, so that $r$ in combination with another consonant may be found in rimes with that consonant. Instances of this are found from the earliest times, thus : Bestiaire, sage : large 7 ; cors : enclus 291 ; Gaimar, ancestre : geste 827 ; for Adgar certes : estes is in the Gregory legend 1468.

In Agn. texts $r$ and $l$ are often found in rime as Bestiaire, nature: nule 103; Gaimar, apostoile : Theodorie 1387; Adgar, cler : alter 30.95, while elsewhere he only uses the form altel (: el 2.93); St Gilles, apostoile : estoire 561. No clear case of this exists in our poem as the rime mariners : juvencels 883 is best emended as in the footnote to the text.

[St., pp. 210-5.]

$\S 29$. Labials.

There is little to note about this group of consonants; $p$ is inserted

2 For this reading ef. $\$ 53,1, B$. 
in Dampnedeu (cf. $\S 19$ ); $b$ after $m$ has disappeared in our text, e.g. colum 1040; $f$ remains in the orthography before flexional $s$, as cerfs 558, nefs 868 ; $f$ final remains in tref 275 and in the rimes nef : tref 945 ; belif : restif 949 . For rimes of -um, -umb- see Walberg's edition of the Bestiaire, p. lvi.

[St., pp. 219-20.]

\section{$\S 30 . \quad$ Nasals.}

Final $n$ and $m$ are not separated in the rime; non < nomen: gueredon 7 ; : compaignon 419. This is, however, not very conclusive as a distinction is made in the Comput, where un and um are confused but not $n$ and $m$ preceded by other vowels. The distinction is perhaps only a chance one since in the Bestiaire crestïen rimes with l'em, while Gainar has faim : demain 325. Adgar would seem to treat these consonants as does Philippe in the Comput but Suchier-Reimpredigt, p. lii-attributes this to pure chance.

In the group $r \cdot n$ as ivern 267, $n$ disappears in the rime, e.g. $j u r$ : blanchur 597 ; tur : dolur 63 . This change is noted from the earliest times; though in the Comput the rimes are not quite conclusive, in the Bestiaire we find jur : honur 249, in Gaimar jur : seignur 319 and in Adgar jor : salveor 3. 7. Satisfactory proof may often be lacking as is the case for Sainte Marie and the Reimpredigt. The change is early on the continent, Chrétien de Troyes has retor: amor in Yvain 6511. Before labials $n>m$ as emfler 860 ; empensé 632 ; it seems to have disappeared from wydas =windas in St Gilles 803 etc. (cf. Chaucer, windas, see Skeat's Glossarial Index). $N$ mouillée is written -gni- when medial as seigniur 651 or $n$ as remaine 725 ; $n g$ when final as lung 275 (for various spellings of $n$ mouillée see Hildebrand in $Z f r P h$. viii 321). In compounds of cum, $n$ frequently disappears before $v$ as cuvint 426 , couveite 445 .

[St., pp. 215-9.]

\section{$\S 31$. Dentals $t, d$.}

Dentals isolated between two vowels have disappeared as is shown by the following rimes: mie : vie 11, vie : amie 75 , amie : hunye 441 etc. With final dentals matters are not quite so simple in Anglo-Norman. The isolated dental become final in French has been lost in our poem in

(a) substantival and verbal roots :

fei < fidem : lei < legem 423; cri : ci 1119; crei : rei 255 . These often rime with themselves as $v e i<$ vido for video $:$ de $i<$ ditum $=$ digitum (cf. Adgar 17. 949). 
(b) terminations, é $<$ atum; $i<i t u m$ or palatal + edem; $u<u$ utem, utum :

Dé : overé 1367 ; gey : di (< dico), nerci : issi 649 ; veu : fu 927. (Abstract substantives and the past participles often rime among themselves as in the ll. 21, 93, 95, 129, 143, 153 etc.)

(c) preterites in $i<i v i t$ :

entendi: autresi 1357 ; fini: issi 351 ; nasqui : si 187. (Preterites of this kind are also found in rime with past participles: as servi: trai 695 and also preterites, servi : guerpi 57.)

(d) preterites in - a, -at rime only with themselves or with a future: alat : enmenat 411; deliat : deliverat 1407; mustra : verra 1423. This proves nothing however as other rimes in $a$ are rare, practically only $l a$ and $j a$.

(e) strong preterites and other words with fixed $t$ rime with themselves :

mist : cunquist 1439 ; fist : dist 1263 ; vait : ait 554 ; aït : dit 1589 ; petit : lit 495 ; plait : mesfait 111 . In line 904 we find the form plai (cf. plait above), this is rare (see Godefroy) but certain Norman texts have it in rime with words without fixed $t$, as plai: delai in Chronique des ducs de Normandie 16252 (for other instances, see Walberg, l.c. p. $\mathrm{lx}$ ).

$(f)$ There is an isolated case of $-s t$ riming with inchoative present: ist< exit : empourist 691 (cf. Adgar, nurist : dist 17.669 and $\S 59$ ). Esperit appears normally in rime with fixed $t$, e.g. vespit 463 ; in Adgar the word is in rime with eslit 6.154 and with delit 6.309 .

This would prove that final $t$ is lost. If the $t$ is silent in the cases above mentioned, a fortiori will it be in -et<-at; although we may admit cases of hiatus yet the cases of elision are regular and prove that the $t$ was entirely lost by the time our poet wrote.

When we compare this phenomenon with other Agn. texts we find:

Philippe de Thaun, Comput: one case of $t$ riming with fixed $t$ but in a line that is not well supported by MS. evidence; it may be a case of analogy.

Bestiaire: no cases.

Gaimar: some cases of preterites in -i, e.g. partit: dit 2514. These are best explained as analogical forms, but it should be noted that certain verbs in $-i r$ show a tendency to join the weak class.

Adgar: no cases.

Sainte Marie: instances only with weak forms as $(f)$ above.

The rime païsant : manant 18. may be compared with tyrant, 
parchment, ancient. Wace, Ambroise and Gervaise and other continental writers admit these forms which are due to analogy with the ending of the present participle and words like enfant. [St., pp. 221-4.]

\section{$\$ 32,33 . \quad s, z$.}

The distinction between $s$ and $z$ (Latin $t+s$ ) at the end of words is absolute ${ }^{1}$. Rimes in -is, e.g. quis : ris 477 ; mis : amis 671 ; assez : entendez 113 etc. are all pure. There are only two apparent exceptions respiz : envis 459 , delez: pres 323 , but these are clearly mis-spellings; envis is for enviz (<invitus) : marriz in Benoit and with pluriz in St Gilles 3240 and pres. is for prez (<pratos). There are one or two instances of confusion in the Bestiaire but Adgar', St Gilles, Ste Marie, Le Donnei des Amanz and St Richard are all alike in making the distinction.

The $s$ before consonant is silent for our poet; this is proved by the following rime in -est, i.e. fest (= fait) : plest 197; other rimes are not quite satisfactory; dist (for the past participle) : fist 217 is not conclusive as it occurs in (B) and dist: respit 499 is probably a mistake for dit. Other rimes are the strong perfects in $11.111,1337$, cf. $§ 31$, while the rimes in -est, viz. est : cest 543 ; est : prest 571 , prove nothing.

For the scribe the mutation of $s$ is proved (i) by the omission of $s$ as in acemée 171 and when final in for 774, 1003 (cf. St Gilles 914); (ii) by its insertion when not authorised by the orthography, e.g. aturnast 1099, mestez 436, neste 353 etc.; (iii) by the characteristic Agn. change of $s+l$ to $d l$ in madles 47, entremedlé preserved in English meddle $<$ medler $<$ mesler.

As to the spellings we find $s$ or ss where we now write $c$ in purchasé 477 , issi 483 and also $s c$ for modern $s s$ in richesce 167.

The outcome of -itium is -ise in our poem, e.g. servise : guise 1245, : eglise 1301. The former pair is also in the Bestiaire 2269. On the continent we have servise : aprise in the Cligés 1779 and with esprise in the Enéas 1269.

[St., pp. 224-30.]

$\S 34 . \quad c=\mathrm{k}$.

This sound is represented in our text by :

(a) $k$ in kar 110 etc., illokes 875 .

(b) $q u$ in quor.

(c) $c$ in iluec 617, iluc 1008.

[St., p. 231.]

1 The rime sëus (cf. $\S 43$ iv $(b)$ ) : vëus 561 is a poetic licence; the word sëus is rare; I have only found it once in rime in Agn., viz. Simund de Freine : seïs : fus (St Georges 1044).

2 In the Gregory legend we find : enfes : enpensez 1576, while Gaimar has purpens : denz 181-2; feiz: reis 923 and some dozen further cases of confusion. 
§35. $q u$.

The spellings $k$ and $q u$ are found indifferently: thus we have $k y$ 101, kant 108, unkes. 191 (but not unques) and qui 1178, que 406, quant 99, quanque 164.

[St, p. 234.]

$\S 36 . \quad c[\mathrm{ts}]$.

This sound coming from $c$ followed by $e$ or $i$ or from $c j$ or $t j$ preceded by consonants other than $s$ is retained here, as in ciel 8 , comence etc. The neuter pronoun is ce, ceo (cf. $\S 50$ ). The spelling -sc- is met here as elsewhere (e.g. Gaimar, Adgar, Simund de Freine etc.) as in manascer 703.

[St., p. 233.]

$\S 37 . \operatorname{ch}[t]]$.

This sound comes regularly from $c+a$ or $p j$; the first of these sources is represented by chant 306 ; chef 64 ; chemin 1261 and-with the spelling sc-by seneschal 1469, meschines 800 ; and the second by sachez (cf. $\$ 54)$. Certain words occur in Northern French forms: desaka 289 ; saké 932 ; secke 353. As is common in Agn. the present subjunctive of saveir is sace (cf. $§ 55 \mathrm{III}$ ).

[St., pp. 235-6.]

§38. $g[\mathrm{~g}]$.

The $g u$ coming from Germanic $w$ is written $g$ or $g u$ before $a$ and $g u$ before $e$ or $i$ : gages $(=$ vague=wave) 861, also Énéas 246 ; garder 1688; garrir 1090 ; garisun 1234; guerpist 7. Original $\mathrm{cr}>$ gr here as elsewhere, graventa 292 ; granter 1214.

[St., p. 236.]

$\S 39 . \mathrm{g}\left[\mathrm{d}_{3}\right]$.

We find both $g$ and $j$; the former generally before $e$ and $i$ as gesir 299 , geta 1241 but only jekes 191, and medially, targié $576 ; j$ is preferred before $o$ and $u$ as joïr 199, jur 301 but gal (for jal) 306 .

$\S 40 . \quad h$.

[St., p. 237.]

Aspirated $h$ is retained in haschie 1222, hernaschant 913, hors 561, huchent 540 , hurte 600 , and $h$ appears in certain words when it should be absent: herbe 192, houre 861, hui 334; we have abit 654 and oure often.

[St., p. 239.]

$\S 41$. Double letters.

Anglo-Norman scribes are especially fond of double letters, our text presents the following:

ll apellé 128, 636.

rr dirrai 376, 986 etc.; querrez 67 ; guarriz 1175; irra 250; verrai 1286. 
dd redde 587.

cc succurs 294.

ss essample 100.

[St., p. 239.]

$\S$ 42. The article.

The nom. sing. form $l i$ is very common and is retained even when the noun has lost its distinctive nominative form, e.g. li tierz jur 330; li pautener 1356 ; it is also found with elided $i$ before nouns beginning with a vowel as $l e m$ 94. In the pl. masc. $l i$ is also very common but the $i$ of this form for no clear reason is not elided before a vowel ${ }^{1}$. For the article in combination with $a$ and de cf. $\S 28$.

A few remarks may be made on the syntax of the article:

1. Nouns are frequently found without the article, when the noun has a general sense as a rei 426 ; apres saluz 326 , cf. too 1l. 836, 876, $1303,1309,1330,1432$; and after certain verbs, e.g. hurte chival $600^{2}$.

2. With parts of the body, side by side with the use of the possessive adjective, e.g. $667,670,817,818,825,925$ etc., we find as piez lur chiet 1361; les piez li ad desseelez 1409.

3. As a demonstrative ${ }^{3}$ :

Seinte Osith fu le jor alée 797 = that day.

A l'aube del jur la matinée $269=$ that morning.

The use of le jur seems fairly common in Old French as: Cel jor $i$ fu Looïs alevez, E la corone mise desus l'altel; Li reis ses pere li ot le jor doné. Cour. de Louis 45-7. His father had given it (sc. the crown) to him that day. Further in Agn. Gaimar: Franceis, Engleis le jor perirent 5418; St Gilles has several instances: Male guarde firent le jur, Quant il perdirent lur seignur 3661-2 and also 11. 719, 2640, 2797; Haveloc: Malement lur avint le jor 110. The persistence of the demonstrative force in modern French as shown by the phrases de la façon (e.g. Est-ce de la façon que vous voulez l'entendre, Molière, École des Femmes, iv 4), de la sorte, pour le coup, à l'instant, etc. has been pointed out by Tobler, Ver. Bei. ii p. 4.4 .

1 Cf: Rydberg, Zur Geschichte des franzïsischen ə, p. 420 seq.

2 Cf. Fredenhagen, Über den Gebrauch des Artikels, Halle 1906, p. 48.

3 Brunot, Grummaire historique I, p. 232, remarks that the primitive nature of the article continued to be felt in the sense that it could take the place of the demonstrative and quotes : Ço dist li reis: 'Al Jesu e al mien!'= for that (sc. the honour) of Jesus and my own (Roland 339 ; cf. too 3145 ibid.). 


\section{Declension.}

\section{$\S 43$. Nouns.}

We shall consider first of all the musculine nouns and shall place in a first paragraph (i) correct forms assured by the rime; in a second (ii) such forms as are rendered certain by the scansion or are correct in orthography; in a third (iii) those which may be easily altered without any detriment to rime or scansion; and in a fourth (iv) such as are proved incorreet by rime or scansion.

i. (a) Nom. sing. guez (: passez) 603; sire (: desire) 445; li reis (: meis) 625, (: Engleis) 131, 140.

(b) Acc. sing. barun (: non) 743; seigniur (: creatur) 4.

(c) Nom. pl. ordiné (: entré) 1251 ; pasturel (: mantel) 332; also pä̈sant 185, seigniur 1269 , sergant 1549, parent 379 , barun 582 .

(d) Acc. pl. chiviaus (: beaus) 26 ; also ordenez 659 , sëus 562 etc.

ii. (a) Nom. sing. li cerfs 558, 581, 584; li fous 1342 ; li paens 140 ; li pauteners 1399 ; li seneschals 1399,1551 ; li sacrestains 1110 ; hom 181, 586, 717, 1130 etc.; li reis $61,444,475$ etc.; li marbre 912 ; li gages 1634 .

(b) Acc. sing. le cerf 580 ; creatur 3 ; mund 15 ; ivern 267 ; faucon 1040; barun 483; conte 743 ; sanc 142 ; home 180, 1558 etc. etc.

(c) Nom. pl. li chanoine 1198, 1269 (final $e$ elided); li chien 567 etc.; li brachet 565 ; li paen 802 ; li seigniur 1293 .

(d) Acc. pl. chivalers 395, 439; seinz 38; enfanz 141 etc.

iii. Add sign of nom. sing. to fet and defet $707-8$, change les chanoines to li chanoine 1483; omit $s$ of compaignons and in line 962 change ses nons to sun non; the change seems authorised by the fact that the adjective is in the nom. pl.

iv. (a) Acc. for nom. peil (: veil) 689; vent (: -ment) 1038; compaignon (: non) 420. There is some excuse for this violation of the rule for peil; the ending -eil is rare in Old French and has been 
replaced except in peil > poil while veil has early disappeared (it is retained in English). Other words feeils<fidelis, chameils<camelus have undergone suffix change, cf. Suchier $§ 65$.

(b) Acc. pl. for nom. peres : meres 23.

We may add a few indeclinable nouns: sëus (<segusius) 248; mes 318 ; sens 248 ; cors 348 .

It may be said then that the author is surprisingly accurate in his use of the Old French declension. There are of course numbers of words which might be corrected and to which no reference has been made, they may well be attributed to the scribe; such are colum[s] 1040; li seneschal[s] $1611 ;$ li $\operatorname{cerf}[\mathrm{s}] 588$ etc. etc. It does not seem plain whether finement (: prent) 37 is an infraction or not, there is no other instance of a Latin neuter in -ment in our text. The forms ancestres 22 , prestres $417^{1}$ as well as compuingnon 420 can hardly be considered as incorrect for Agn., the first and the last appear with the single form for both cases already in the Oxford MS. of the Roland. There is only one instance (1. 1377) of an infinitive used as a noun in the nominative case in rime and it is without $s$, the same archaism is found in the Comput 985, 2791; the scribe has added an $s$ in 1. 942; for other cases see 1l. 413, 618, 715, 1192.

There is little to remark about proper names; none appear in the nominative case in rime except Editz (: diz) 233. This is one of those poetic licences with names so common in Gaimar. For nouns of the Latin third and fifth declensions it seems impossible to say whether they take an $s$ or not. There is little to be said of feminine nouns. In Old French there are few feminine nouns belonging to the class with varying stress. From sóror, sorórem we have nom. suer, acc. serour, pl. serours, but owing to the frequent use of the form suer as a nominative of address, serour is early lost and a new pl. suers even formed. As early as the Roland suer (1. 294) in the accusative is found in an ué assonanced tirade. Further serour is found as a nominative and even with an added $s$. Similarly are treated words denoting persons having a nom. in $-e$ and an acc. in -ain as none, nonain. Our text seems to offer instances of these changes. Thus in 1. 223 the rime with amur seems to indicate that the form seur should be changed to serur; it would have the further advantage of correcting the scansion; while in 1. 242 the form serur is assured by the rime with ducur and ele may very properly count as one syllable only; $\operatorname{cf} \S 5$ ii $(\beta)$. The form serur

1 Another reading is suggested in $\S 44 \mathrm{~A}$ iii. In Adgar we find proveire as nom. (:aneire) 10.36 and as acc. 13.56. 
seems particularly suitable as it is a later form and these lines are in (B). In 11. 1095, 1217, 1229 suer (notice the older spelling) is correct, while in 1.1220 the adoption of the form seror would enable one to alter the incorrect porte (cf. $\S 55 \mathrm{i}$ ) and would bring the accented final syllable seruir into what we may assume was its position intended by the poet, viz. the fourth sylliable. In l. 371 we have nonain as nominative. In our text the oblique cise can still be used in a limited sense for the genitive, particularly in such stock expressions as la Deu ancele 49, 635; les seinz Deu 38; Deu enemis 963; pur Deu amur 40, 660; also before proper names as le jur Seint Michel 1105; and when the genitive expresses a person, les plaiz l'evesque 14.58, 1470.

There is little to remark as to gender. Honur wonld seem to be feminine but the evidence of the adjective trespassable (: estable) 17 is unconvincing. Fiertre appears both as masculine and feminine 1491, 1495 ; in the only other case that I have met it in Agn. (St Richard 1639 ) it is masculine; by analogy probably with châsse it became and remains feminine.

\section{$\S 44$. Adjectives.}

The adjectives may be treated in the same way as the nouns and divided into (i) such forms of adjectives and past participles as are correct judged by rime and scansion; (ii) such as may be considered correct from scansion and orthography; (iii) such as may be corrected without detriment to scansion or rime; and (iv) such as are proved incorrect by rime or scansion.

A. Masculines.

i. (a) Nom. sing. -ez<-atus, iz<-itus, -uz<-utus are assured by the following rimes: aprestez (: entendez) 29 ; passez (: guez) 604; evaniz (: partiz) 622 ; restuz (: venuz) 615 ; and also in the following: 493, 720, 912, 987, 1351, 1352, 1403, 1404, 1553, 1554, 1621, 1649, 1650.

(b) The nom. pl. without flexional $s$ is assured by the following rimes: alé (: trespassé) 31 ; lé (: mangé) 491 ; avenu (: vertu) 82; grant (: siwant) 567 ; seur (: jur) 864; also the 11. 115, 207, 607, 623, $769,792,885,888,939,941,954,1010,1015,1016,1017,1018,1050$, 1057, 1083, 1084, 1236, 1340, 1381, 1382, 1484.

ii. (a) Nom. sing. fols 20 ; bons 37 ; gariz 1635.

(b) Nom. pl. luintain 187 ; lé 379 ; venu 767 ; also 956,1168 , 1257, 1484, 1512.

iii. The following could easily be corrected :

bon 2; fort 61,279 ; 138 ; lung 275 ; blanc 558 ; institute of Media 
venu 548,584 ; conissant 970 ; contenu 1371 ; sein 1534 : the lines $417-8$ might be emended Proveire esteient ordiné, $E$ seint evesque puis sacré.

iv. The following are incorrect according to rime or scansion:

Nom. sing. puissant (: refusant) 61, 673 ; fort (: port) 609; restif (: belif) 950 ; and also $100,963,983,1346,1546$ and in (B) parfund (: punt) 277 ; bruiant (: despant) 279.

After a neuter subject, whether expressed or not, the attributive adjective is normally neuter, i.e. without flexional $s$, in Old French, but the feeling for the neuter is too vague for this to be an invariable rule; the masculine is often found (cf. Brunot, Grammaire historique, I, p. $223 \mathrm{C})$. . The neuter seems to be preferred in Agn., e.g. Bestiaire: E cuvenable esteit 71 etc.; Gaimar :...pur ço est dit (: murdrit) 4053; co est dreit (: esteit) 5112 etc.; Adgar: ceo n'est dreit (: toleit) 8. 133; St Gilles: il es dreit 2091. The examples for our text are in lines 58, 851 (cf. §59), 1369.

B. Feminines.

Taking the feminine adjectives and participles in the same order there are few infractions of the ordinary rules of declension.

i. The adjectives in -abilis are correctly trespassable : estable 17; grant (: tant) 536; : apurissant 819 ; other lines showing correct forms are: $69,73,127,170,362,363,367,368,387,421,439,443,462,797$, $810,826,1117,1167,1215,1262,1375,1389,1411,1651$.

ii. The existence of forms as grant is assured by scansion in 1l. 138, 806, 1063 and lasse by 11. 1166, 1224.

iii. Changes could be made without detriment to the text in: granz 16, mené 377 ; also in 11. 464-5, 651, 829, 1106, 1167.

iv. Except for tele (already common in the twelfth century) all the infractions are found in (B), e.g. repairé 231 ; remansu 268 ; irascu 312.

$\$ 45$. Agreement of the past participle.

The rule for the agreement of the past participle when the object precedes is observed in the following cases assured by the rime: 444 , $481,678,809,1118,1201,1202,1222$ and with the object following in line 421. (This may be because of the rime.)

The rule is broken in the following cases in rime: 148, 688, 1513, 1514, 1516 and in (B) 285, 288. Lines 200 apris[e] and 719 fet [e] could be so emended.

The non-observance is sometimes convenient especially in (B) and (A) as rimes otherwise impossible are thereby secured, e.g. 1513-4. This is common in thirteenth century Agn. poems, cf. St Richard $\$ 50$. 
$\S 46$. The past participle with reflexive verbs.

Only one case of agreement of the participle with the subject in the masculine seems to occur in rime: s'est partiz 621 ; but there are a large number of feminines, e.g. 269, 302, 409, 440, 709, 829, 1133, 1136, 1258. There are other cases correct in the interior of the line, e.g. 617 , while many others could be emended by the simple addition of [z]; e.g. 496 , $570,978,1359$; one case only of non-agreement seems assured by the rime, viz. 1325 .

$\S 47$. Adjective.

A. Possessive (possessive pronouns are also included).

The ordinary weak forms are all found and the following alone are noteworthy : si (nom. pl.) 1529 ; le men 982 ; le mien 437 ; la sue 505 ; un lur 1321 ; les seens 1542 .

B. Demonstrative and indefinite.

Our text has nom. sing. cil 140,512 and cist 1441 side by side with icest 1160 and cest 1090. 'The correct forms are doubtless those of the poet but none of them appear in rime. Cist is found in line 582 as nom. pl.; the acc. pl. is ces; we may notice also cil autre as nom. pl. 930 ; itel and tel as feminines 928, 1024, and the use of the definite article with these adjectives as des ces 763 . For the indefinite we note aukes de...231, 448 etc.

$\S 48$. Pronouns-personal and reflexive.

The strong-accented forms of the personal pronoun are found :

i. After prepositions : par mei 244; pur li (masc.) 58; a ly 497; a $l i$ (fem.) 244; pur lui (fem.) 196; ou li (fem.) in rime with ci 806 ; also 11. $670,1165,997,149$.

ii. Before an infinitive: sei fere 965 , also $530,534,641,1168$; further after an infinitive: pur prendre sei 721 .

iii. After an indicative: purpense sei 721 ; also the weak form: e traveillent se which should perhaps be emended to sei 1193. (Cf. Bestiaire: Deus la furmat e concut lui 3105.)

iv. After a past part. and before a finite: $E$ estrussé li ad...1111.

v. As a disjunctive : $l i=$ elle, cf. $11.282,361,636,800$.

vi. With reflexive verbs instead of sei as de li mover 1070 .

It is doubtful whether this form may be used before a finite verb. The Agn. MS. of Sainte Marie l'Égyptienne 991 has e li turna ver's Orient but this may be an incorrect reading, although it turns accurately the Latin: ad Orientem conversa; the continental versions have ele garda or esgarda. 
The pronoun ele has often the syllabic value of $e l$ ', e.g. 312,319 , 1246 and is sometimes so written, but oftener not.

All the weak forms are found, $l i$ seems to be the usual form for both masculine and feminine; lui only appears once, line 196 . The pronoun en appears in its modern use in line 387 and also in its Old French sense of ' in the matter,' e.g. 714, 998. In line $272 u=\dot{a}$ laquelle and in line 694 $i=l i$ (cf. Brunot, Grammaire historique, I, p. 226, Bestiaire: Quant diable at tempté Saint ume e espruvé Ne mal n'i pot truver, 1167-9 Jordan Fautosme: Fors la cité de Lundres, $u$ nul ne set sa per, Chronique 912 and Modwenna: A tele vus cumanderai, $U$ jeo tres bien m'afierai 527-8). The $i$ of $l i$ is elided in line 286 (B), cf. St Richard $\S 52$.

The syntax of the pronoun in our poem is no longer that of the oldest texts; whereas any of the pronouns may be omitted as subjects, all may be used: There is probably a slight difference, thus in 11.1143 , 1145,1146 there is some stress implied by the sense which is quite absent in $11.1147,1149,1150$. Instances of omission are very numerous, e.g. ll. $660,757-60,917,1004,1100,1141,1175$; instances of use $661-2$, 968,1327 etc. There is a good instance of a strong use of $i l$ in line 686 and of $i l$ as disjunctive in 493 .

There are two cases which may be discussed separately. In 11.698 and 1108 we find the weak forms of the pronoun used after an infinitive and the infinitives are complemental depending on a preposition. The common usage here is to employ the strong form which should precede the infinitive. In the first of these two cases the pronoun is perhaps spurious, the line has a syllable too many and that in a portion of the poem where the scansion is on the whole accurate; it may have been introduced from the following line; further the MS. has a sign ? which is employed elsewhere to indicate a pause in the sense, e.g. l. 752 after servir. We propose to emend by omitting the la. In the second case the position may be due to the desire of the poet to avoid hiatus of the final $e$ of faire with initial $i$ of iluec. To explain the use of the weak form the poet may have had in mind another construction, viz. the use of the weak form with a finite verb having an infinitive complement; this form would, and generally did even till the seventeenth century, precede the finite verb but it might immediately precede the infinitive; thus in the Bestiaire in two consecutive lines we have an example of each construction: Nel volent esculter, Volent le desturber 1715-6.

The use of the pronoun after the infinitive is a peculiarity of N.W. texts. According to Rydberg, o.c. pp. 587-95, it is confined to those 
districts that to-day form the départements of Eure, Seine inférieure, Calvados, Manche and Seine et Oise, i.e. nearly all Normandy and a part of Ile de France. It is natural therefore to find cases in AngloFrench works. liydberg quotes a large number of instances but in most of these the strong form of the pronoun is preferred.

The use of the weak form of the pronoun after a finite verb seems to be rare; I can only find in my notes two instances, viz. Tristan of Béroul laidissent la 775 ; and Roman de Troie: Honora les de grant maniere, 1204.

$\S 48 a$. The pronoun with reflexive verbs.

Whereas in Old French the number of reflexive verbs was greatly increased by the inclusion of verbs that were intransitive in Latin, there was a tendency to omit the reflexive pronoun and to render the verb intransitive; this was specially the case with the present participle and the infinitive. Numerous examples are quoted by Brunot, op. cit. p. 237 ; cour text offers examples in 11. 939, 1425-7. The verb which seems to have most easily dispensed with the pronoun is se lever; our poem offers the following instances: Bien leva devant (corr. ainz?) le chant de gal 306 ; Et par matin est tost levé 1359; $E$ cele est par matin lcvée 1411. Examples are very numerous; thus: Bestiaire : Kar se culchiez esteit (the elephant) Par sei ne levereit 1545-6. Examples do not seem very plentiful in Gaimar ${ }^{1}$ or Adgar ${ }^{2}$ but St Gilles contains many, e.g. Par la cité leve le cri 753 ; le soleil leve 916 , also 2739, 1379, but $I l$ s'en levat 622 . Sainte Marie has similar cases and so too Marie de France : Li chevaliers cuntre els leva (Guigemar 769 and ibidem 673, 869 and Deuz Amanz 214), also Haveloc 708 Le cri lieve en la cité; ibid. 903 Haveloc est levé en piez. In the Roland we find Isnelement sur lor piez releverent 3575 and Crestien de Troyes, Erec: Quant a lor eise orent sopé Et des tables furent levé...501-2. There is a full discussion of this phenomenon as far as the seventeenth century is concerned in $\$$ 60-61 of Haase, Syntaxe frunçıise du xviie siècle.

$\S 49$. Pronouns-relative and interrogative.

The scribe prefers the spellings with initial $k$; we have many instances of $k i$ or $k y$, e.g. 2,39, 101 etc. but very frequently ke stands for the nominative; this form must often be due to the poet since this

1 I remember no case with lever but we find Quand il turnad de la cuntrée (cf. 11. 3675, 4013), compare with Cil s'en turmad 4513 (cf. 4237).

2 I can only recall Establi a sei itel uq Que chaune nuit leva sus, Si ala puis en cel nustier 18. 31-3. 
$e$ is elided with the following vowel, e.g. 1317, 1375 (not elided 1092); $k i$ may stand for older cui 112,830. The poet uses the relative que in the feminine 805 but this may be only a spelling and of no importance. The neuter is ke 1141 and also kei 312,319. Note too ceo que 406 referring to a phrase.

\section{$\S 50$. Pronoun-demonstrative.}

The forms are fairly correct: nom. sing. cil 9, 12, 453, 1171; incorrect cel 237 (B); non. pl. cil 1043, 1047, 1123, 1455; acc. pl. ceus 21 ; fem. cele 1177. The poet is fond of the longer form icest which is twice in rime 544, 730. We. have also cesti as acc. 626 and ceo is common; for the compound demonstrative we have ly qui 569.

\section{$\S 51$. Pronoun-indefinite.}

The following forms occur: l'em 94, 164; l'en 372 ; l'un 955 but regularly (cf. $\S 42$ ) li un...li autre 542 ; les autres 919 ; chescun (never with s) 926 etc., with verb in pl. 1028; aucun 1173; plusurs 187 as nom. pl. in (B) but not assured; for Latin totus the forms vary but the nom. pl. tuit is in rime with bruit 999 ; amdeuz and amedeuz occur in (B) 288, 341 ; tele 55 assured by scansion; mult de 1299 and aukes de are common.

\section{\$52. Adverbs.}

The adverbs are well represented in our text: petit 165 together with poi 18; aval and amunt 869 ; sus e jus 624 ; guerres 67 and gueres in the sense of nothing 680 ; leus 323 (a form also found in the Corpus MS. of Sainte Marie); meuz (= plutôt) 1365; a plein (cf. $\S 14$ ) and the Agn. form sovente feiz (cf. $\S 59$ ). As adverbial complement we may note mot ne sona 903. An interesting word is enteins (cf. $\S 59$ ). The adverbs in -ment are mostly in the older and correct forms as: comunaument 804 ; cruelment $796,807,986$; delivrement 556 ; erranment 986 ; estrussement 499 (this adverb is derived from estrossé and should therefore be estrosséement, such seems to be the form in Erec 5592); forment 507 ; leaument (not lelment as often in Agn.) 835. Dreit and tut dreit are found but not abusively as is often the case in Agn. (cf. St Richard $\S 57$ ); tut and trestut are common 558, 658, 606 and so too are a tut 780 and del tut 829, 864. Note too aukes 887.

\section{§5: Conjunction.}

The question of the conjunction in our poem is almost exclusively the syntax of que as few compound conjunctions are to be found in it; 
$s i$ is still used though sparingly for et, e.g. $119^{1}, 527$ and also ne 168 but $s i$ is already for if at least for the scribe. The syntax of que is one of the most interesting chapters of Old French grammar ${ }^{2}$; we shall endeavour to sum it up as concisely as possible, adding too the examples where no que appears.

I. With verbs denoting intellectual activity.

i. knowing, believing.

A. With the indicative in the dependent clause:

Sachez ke la pucele...esteit bele 169 ; cf. 1663.

Purpensa sey...ke rien ne valut sa dolur 721 ; cf. 895, 897.

With infinitive: Cuida son livre aver.receu 287 (B).

No que: Tres bien suvez, Vers Dampnedeu mespris avez 1561.

Quide (ke) deable l'en $a$ traï 696 .

B. With the subjunctive.

Sin crei...ke seinte Osith gré le squvast 119-20 (cf. §59).

Lors ke neée fust suscherent 239.

Ke conisant seit...ke...ne seum...perilié 970-2.

ii. perceiving.

A. Quant la dame entent Ke ne volent faire nient $663-4$, also 1279.

Quant cil veient ke n'ad mestier 951, also 723, 1602.

Bien...ad aparceu K'ele $f u$ dolente 311.

No que: Kant veit ne puet estre muée 723, also 1588.

B. Entendez...ke en puissez aver aïe 123.

S'il veit ke el estre ne peust 727.

iii. declaring.

1418.

A. Dirrez (ke) granz biens i puet trover 245 , also $250,304,631$,

E...voé unt Ke chescun ferunt porter 1027.

Il resona ke a lui se vout...468, also 469 .

No que: Bien l'os dire, fols est pur veir 20, also $499-500,515,703$, 707.

Ceo signe (ke) de joie volent trere 1496.

B. Ke dunc fust nonein ne di pas :371.

Unk cele nef ne fist samblant k'en ewe fust...921. iv. Impersonal verbs.

A. Un jour aveneit...que Modwen aleit 825 .

1 119. In this line the si en of the MS. is to be read $\sin$.

2 We shall follow throughout the able statement' of its importance as contained in Mr Ritchie's Recherches sur la syntaxe de li conjonction 'que' dans l'ancien français, Paris 1907. 
Acune feiz avint...ke grant tempeste out...859.

Ke deit ke nostre nef ne vait? 934, cf. $§ 59$.

B. When the principal clause is negative or hypothetical the mood is the subjunctive; there are no cases of this in our text.

II. With verbs denoting emotion.

A. Old and Middle French prefer the indicative mood and we find: Molt se merveille que ge puis tant targier (Cour. de Louis 1108) and $\mathrm{Je}$ regrette de tout mon cueur que n'est icy Picrochole (Rabelais). AngloNorman, however, seems to prefer the subjunctive though some instances of the indicative are to be found, e.g. ("est merveille ke jo m'en tais (St Gilles 116), also ibidem 245, 2877 and Ce peise mei ke jo sui vive (Ste Marie 414). Our text offers no instances of the indicative.

B. Edith s'est emerveilliée Ke fëist Osith tant targer 302.

Li chanoine s'en vunt plaindre a Deu...Ke seit eus vengant 1483.

Pur estre grevé Ke de havene seient jeté 939.

N'estut doter Quel jur deive del siecle aler 35.

III. With verbs denoting volition.

i. will, command, prayer, obligation.

A. The only case of the indicative is one in which no que occurs.

Tant a requis...Le dreit chemin li unt mustré 1188.

B. Jeo voil ke le veil me donez 645 .

A seinte Osith a graanté Ke remaine tut autresi 723, also 1294.

Suffir ne vout K'ele seit al mustier 1112, also 1505.

Deu a purvueu issi Ke guerisun eies 1153.

Comandent...K'il augent tut a avirons 935.

Covient...Deu prier K'il nus pardoint icel pechié 1008.

Covent ke jeo meste mun don 736 .

So also with demander 1184; orer 120 ; crier 1127, 1232 ; reclamer 584 ; requerre 176, 403, 659, 1143, 1296, 1386, 1620; conseillier 208 and less usual phrases as crier merci ke...471; metre en preres ke 341-4; prendre penitance ke...966; estre en aïe ke...1685.

No que: Deu unt requis... Mes ne seient en tel baillie 1024, also 403-7.

ii. preventing, forbidding.

1. Requert Deu li purgart...ke ne perde 407 .

2. Metrai mon poer, Ke ne seie...hunie 437.

3. A seinte Osith ne puet hom mesfaire K'ele n'enprenge 1571.

4. Comanda aler...pur veer Ke a Chic [ne] deusent poseer 1453.

The use of ne seems absolute and as it occurs in exs. 1, 2, 3 I add it in 4 also. 
The indicative is sometimes found when a resolution is expressed: Puis ceste hure mettrai...Tute ma force...ke mes travail n'avrez $11.531-3$.

IV. The co-relative.

The use of the co-relative is not frequent in our text; ço is by far the commonest: Ceo demander serreit folie 322; Mes tut seez de ceo seur ke...1664, or adverbial expressions as L'alme del cors pur poi n'en ist 691 .

V. Que consecutive: que $=$ si bien que.

A. Et par les gerrons...prist Son mantel ke le livre obli 282-4.

Et travaillient sei...ke venu $i$ sunt 1193 and with the expressions si ke, si cum, tel ke, tant ke...

La contraite...plure si ke noiant esteit escuter 1242.

Ele prend tel vengement ke...bien parra...1573.

Tant veit $k$ 'al muster est venue 810, also 1213, 1224, 1326.

No que: Seinte Osith l'a si forment mis...Ne puet...plus estre ostez $1347-51$.

If the result is positive, the verb is in the indicative.

VI. Que final. (The verb is always in the subjunctive.)

Modwen a Edith Osith enveia Ke bien la preist...227.

Le liu Nonnepol est apellé $\mathrm{Ke}$ ja mes en siecle ne seit celé 370 , also 363.

The so-called optative use can be included here; with and without que.

Amis ke Deu vus doint sauveté 327 .

Seinte Osith nus seit en aie...1685.

Deu penst de mei par sa merci 666 .

VII. Que causal.

i. $\quad$ que $=$ weakened car.

Ke Deu ama e Deu servi 57. This use is uncommon in our text and in the example here quoted Ke might conceivably $=$ qui.

ii. $q u e=$ puisque.

Only in the phrase quant est issi where quant replaces que 665, 729, cf. $§ 59$.

iii. que with interjections (the ordinary tense is the pres. indic.). Allas, ke il ne set icest 544 .

$\mathrm{Ha}$ Deus ke Edith ne seust l'aventure. (This is possibly corrupt and should read: $D_{\text {i }}$ ! k'Edith ne sot...but the subjunctive might stand though it is very rare $^{1}$, for the scansion in that case see $\$ 5 \mathrm{ii}(\zeta)$.)

1 Mr Ritchie l.c. p. 74 quotes only two cases of the past subjunctive and very few of the past indicative. 
VIII. Que temporal.

quant).

i. With expressions of time (que is here often replaced by

En icel tens ke cil veneient 1459.

Le jur ke seinte Osith alat, Kant li reys...l'enmenat 411-2.

Meïsme l'houre k'est venuz 1623.

E gueres plus ne demora, $K$ 'il autre feiz la resona 468.

Seinte Osith a ceste houre en pris Ke sur son chief...677.

L'ure quant sunt li chien passé 607.

ii. With compound conjunctions expressing :

- (a) simultaneous action:

Dementers $k$ 'en coste vie fu 195 .

A ceo k'esteicnt haut chantant 1199 (cf. § 59).

$(\beta)$ posterior action:

$A$ ceo ke il ad parlé ci 1591 (cf. §59):

A peine out Modwen sa voiz fini Ke de l'ewe Osith ne issi 351.

(For a discussion of this phrase, see $§ 59$.)

Dekes a Deu s'est si rendue 709.

No que: Hui est le quart jur trespassant, Puis n'en oi mes...317.

$(\gamma)$ anterior action:

De ci ke vint al vendredi 1540 .

Si cum (= aussitôt) Edith aveit veu 309.

IX. Que comparative.

Bons fu ki...aime son creatur Plus ke ne fet autri 4.

Kant sa dolur a fet assez Ke tut put estre alessez 719-20.

Mes plus semblant ne fist la nef $K e$ maison fet le cuchetref $945^{1}$.

Si l'aturne a meuz ke poeit 1099, also 1131.

Meux le vus voil dire...K' autre le vus $d^{\prime} e^{2} . .1365$.

$\mathrm{X}$. Que concessive.

As above with temporal clauses quant competes with que, so in causal, final and concessive clauses cume or cum is often found; this is the case in the only example in our text:

$\mathrm{Ne}$ sai cument...Fors cum Deu le vout...1049.

It would seem here, however, that cum or que should be omitted, cf. $\S 70$.

1 Cf. $\$ 59$.

2 Note that this que stands for que que, English than that. This nse was retained in old French long after it had become impossible to omit que. Grammarians suggest that this should be avoided by using que si as: Il vaut mieux que l'innocent perisse que si toute la nation allait se révolter contre César (Massillon). What I believe to be the oldest instance of 'this is in the continental version of Sainte Maric: Por ce est il plus avenant Que tu le doinses or a moi Que se je le donasse a toi, cf. Ritchie o.c. p:93. 
$\S 54$. The conjunction, contd.

The value of the conjunction for determining the date of a literary work has been suitably foreshadowed by $\mathrm{Mr}$ Ritchie in the work mentioned in the foregoing section. The matter is bound to receive henceforth the closest attention of philologists. It is an additional test to be applied ${ }^{1}$. Unfortunately linguistic tests applied to French works produced in England are always sonewhat unsatisfactory, since they may be at once more archaic and more modern than continental French works of like date. We remarked in $\$ 17$ that some knowledge of an author's upbringing is necessary and this often has to be arrived at by long and not always incontrovertible methods. Thus the Vie de saint Gilles, judged from the linguistic point of view, is perhaps as old as 1150 while certain matters of fact referred to in it place it certainly some quarter of a century later. Applying the test of the conjunction to our poem, we find that no violation of twelfth century syntax occurs in the-main body of the work; it is only in (B) that we find a peine... que which, as is stated in $§ 59$, I have not met with outside fourteenth century texts.

\section{$\S 55$. Conjugation.}

\section{Persons :}

The first person singular takes neither $e$ nor $s$ when these are not authorised by the etymology as is proved by the following rimes or scansion : comant (: puissant) 674; cri (: ici) 640, 1119 ; jur (: seur) 863, also aim 1287 ; os 20 ; vei (: dei, cf. $§ 31) 1602$; crei (: rei) 253 ; di (: oï) 1657, (: si) 1636 ; rent (: finablement) 1292, also promet 527 ; quer 1122 ; enquer 994. The form porte 1220 is probably due to the scribe and the line should be emended as suggested in $§ 43$; yet forms with $e$ occur as early as Brandun (circ. 1120-5) while doins 737 is already in the Roland and the Couronnement de Louis.

The first plural ending is only once written -ums 961, otherwise it is $-u m$ but of course sumes 956 ; the ending -um is many times in the rime but always with itself, e.g. 1005, 1435 etc., 337 (B). The third plural has occasionally -unt (cf. $\$ 5$ iv), e.g. 28,759 etc.

II. Infinitive :

The verbs of the first conjugation are never confused with those of the third as saver: demorer in Beuve de Haumtone 898 except saver : targer 304 in (B), cf. $\S 15$. This phenomenon is common by the middle

1 Thus Mr Ritchie applying this test to the Pèlerinage, considered by many to be an eleventh or very early twelfth century text, doubted that it should be ascribed to a date earlier than the end of the tolelfth century, while M. Coulet (Études sur le Voyage de Charlemagne, Montpellier 1907) (arrived at a like conclusion from other considerations. 
of the thirteenth century and there are some cases in the rimed sermon Deu le omnipotent, e.g. penser : aver (altogether five cases in 122 sixains), but in certain texts as St Richard (circ. 1270) the confusion is limited to the commonest verbs as: aver, saver, ver, poer, valer. There are no instances of this in Gaimar, Adgar or St Gilles. The form obli (: chai) 283 suggests an infinitive oblir; such barbarisms are frequent in late thirteenth century Anglo-French.

\section{Present:}

The third person singular of aler is vait in rime with trait 669 and also dreit 899 , cf. $\S 13$; the third plural of dire is dient 927 . In the subjunctive the verbs of the first conjugation have no $e$ in the third person, e.g. penst 627 ; purgart (: tart) 407 ; pardoint 327 etc. ; aït 1590. Other forms are augent 936 ; donez (i.e. doniez) : targiez 645. The third sing. of saveir is sace but not in rime. This is the normal form for Anglo-Norman; it is in rime with grace in the Bestiaire, in St Laurent, Tristan and Adgar and with place in Ipomedon 2297; from prendre we have prenge 1573, already in the Bestiaire 20 and Donnei des Amanz 74.

IV. Imperfect :

The imperfect ending -out of the first conjugation is only once -finout ${ }^{1}$ (: plout) 212 found in rime contrary to the practice of most Agn. writers; the ending -eit which is often added to verbs of the first conjugation is here found only in three instances: two in (B) 201, 225 and one in (A) 1438. From estre we find ert 267 and estoie 987.

\section{Preterite:}

For $t$ of the weak forms cf. $\S 31$. The preterite of chaïr is chaï and of remaindre remist; this form is already in the Bestiaire but here only in (B); that of voleir is vout 453 and vot 237. The first sing. of saveir is soi 320 . There are no 'dedi' perfects; thus the preterite of tendre is tendi. The form $f u$ is alone used both before vowels and consonants.

VI. Future and conditional :

From doner we have durra 500 and dureient 1474; from laissier (laier) larrum 299; from estre ert 29 ; further plereit 242; parra 1574; the shortened forms of faire, e.g. frai, are common in Agn. since the Bestiaire and so too the trisyllabic forms avera etc.; these last are, however, always dissyllabic according to the scansion.

VII. Imperative :

The only form of interest is diez 328 .

1 This form even is not quite certain; the correct reading might be fin out; the tenses would then be in better agreement. 
$\S 56$. The government of verbs.

Very little attention has been paid to the government of verbs in Old French in the standard grammars; I am therefore contenting myself with a simple constatation of facts as they are in this poem.

aidier vers 121, 126; here the prepositional complement seems rather to belong to avancer or amendement.

apendie de 754 ; usually with $a$ or envers, e.g.

Deus ne fist terre qui envers li n'apende;

Il i (=lui) apent Baviere et Alemaigne. Cour. de Louis Il. 16-7. comander (or mander, cf. $§ 59$ ) followed by infinitive without preposition $577,1244,1451$.

comencer without preposition 498, 275, 697, 777, 834. Cf. Burghardt, Über den Einfluss des Englisclien auf das Anglonormannische, where many cxamples are quoted with $a$, de or no preposition; cf. too St Richard $§ 67$.

donner vers 1693: no instance in Godefroy. Cf. La Folie Tristan (MS. Oxford) 323.

entendre vers 1556, generally with $a$, cf. 1. 87; no instance in Godefroy. mesfuire a 1572 ; the usual construction is with vers; Godefroy $v$ 282.

mesprendre vers 1562 ; one instance in Godefroy viii $203 \mathrm{~b}$; in the Donnei des Amanz 74 with devers.

oir de with infinitive 429 and with entour 89; no instances in Godefroy.

se peiner de 429,618; the examples in Godefroy are of much later date than our poem.

servir a 8,759. This would appear to be a latinism, though classical Latin uses the dative or apud; I have only met this construction in religious and didactic work (cf. Koschwitz, Commentar p. 90, §4). The same construction occurs in Sainte Marie l'Egyptienne 1. 81. Godefroy $\mathrm{x} 669 \mathrm{~b}$ quotes this use in a somewhat different sense.

tucher de 201, 1436. No examples in Godefroy of this use till modern times.

$\S 57$. Periphrastic constructions.

Our text contains many such constructions:

i. aler + present participle 1374, 1501, 1516, 1530, 1535, 1543.

ii. estre + " " $" 317,474,889,1488,1637,1641,1642$.

iii. venir + " " 63-4, 1491.

1 Further Vie de saint Andre, 1. 397, Et s'a mon Deu ne vus servir in MSS. Paris, Arsenal 3516 and Oxford Canon. Misc. 74 . 
The reading l'ad tut refusant 1.62 should perhaps be $l i$ ra... Instances of these constructions may be seen in plenty in mono-rimed tirades of Renaut de Mortauban.

\section{$\S 58$. Voleir with the infinitive.}

This construction with a future sense has been called an anglicism by Burghardt, $l$. c. pp. 57-74. Without a fuller examination of continental didactic work I am not inclined to accept this view, but the frequency of the construction in Agn. is striking; since, however, the main output of Agn. literature is religious, the source should perhaps be looked for rather in late Latin didactic literature. Our text presents: voil + infinitive in $11.65,648,742,981,982,1364$; volum + infinitive in line 1004; voudrai in line 642 and voudrunt line 1673.

\section{$\S 59$. Words and phrases.}

se aveir 1437. G. quotes this in the sense of 'se conduire'; its meaning here is rather the Latin one.

barun =husband 483 , cf. Panuce, published by me in Romania XXXviII p. 421 .

brache $=$ braquet 562 . Godefroy only quotes brachet, ef. 1. 565. Cf. Bestiaire of Guillaume le Clerc 224.

cheminal; see footnote to line 307.

chescum 190; Agn. scribes adhere carefully to the form chescun or chascun but in its adjectival use it is often found as e.g. en chascun endreit-a group of four syllables-where the scansion seems to demand chaque, a form of only rare occurrence in the thirteenth century (cf. Brunot, Grammaire, ii 320).

comander and mander 577, 1244, 1451; the confusion of these two verbs seems to be complete.

cuchetref 946. This word does not to my knowledge occur elsewhere; I believe it is the sail that was used as an awning and known in St Gilles as tialz (see below teolde). Professor Brandin has kindly suggested to me that the line may perhaps have read $K$ 'en maison fet le archetref; I have not found archetref nor indeed architrave earlier than the sixteenth century. In favour of cuchetref may be cited several meanings of couch in the N.E.D. Professor Weekley suggests to read Ke maison fet ne (for le) cuchetref.

deit 934 ; see footnote to line, cf. $\S 53$ iv $\mathrm{A}$.

despire 166. This verb seems to be exclusively Norman and Agn. empaourir 692. This would seem to be a variant of espaourir, cf. 
Pèlerinage 709 where the MS. has aspourie; espoörie is also in St Auba.. 1. 486 and Vie de St Grégoire by Angier, 1934.

enfundré 296. Godefroy quotes enfonder and variant enfondrer. The line should perhaps be corrected enfondrés; cf. $§ 65$.

enteims 157, 939, 1479; according to G. Paris, Introduction to $S t$ Gilles p. xvii this word is not often found after the twelfth century. It occurs in the Donnei 1. 375.

entrussément 499, estrusser 1111 . The adverb and the phrase $a$ estros are very common, but the verb does not appear in Godefroy in the sense it has here of 'to drive out.'

envilir 1517. Godefroy does not quote this form but only aviler and enviler in the meaning here implied. (For en-for $a$ - see $\$ 6$.)

esquieler 1322 quoted by G. under escueillier = scutellarius, a scullery man. I am indebted to Professor Suchier for this hint.

esperital $308=$ inspiration, only as an adjective in $\mathrm{G}$.

frankeleins 490 . G. docs not quote this word; its meaning is evidently a small freeholder.

fanfelue 131s = modern French fanfreluche.

gerrons 282 = the flaps of the cloak.

glu 1331, cf. Godefroy, Supplément.

gré $=$ willingly 119 . G. mentions this use but quotes no examples; I have met no other instance; the line should be emended as in $§ 53 \mathrm{I} \mathrm{B}$.

krenke 290, apparently an English word such as Agn. writers often introduce into their works, e.g. D'ales en engleis dist hom Ely' (Vie de Sainte A udrée, folio $102 \mathrm{v}^{\circ} \mathrm{b}$ ).

menestraus $1444=$ servants; in this sense its use seems to be confined to didactic literature, e.g. Alexis $65 \mathrm{~d}$; Chardry, Sept Dormanz 766.

merir $1674=$ reward; cf. G. v $259 \mathrm{a}$ and the late Latin distinction between mereo and mereor.

ostez 574. Godefroy quotes ostes s.v.; 'exclamation employée pour repousser l'idée d'une chose'; it occurs very frequently according to him in the Roman du saint Graal. If this form is correct, it is interesting to consider it as an imperative forming an interjection and to compare it with the English out! which may possibly be formed on the same model.

overaine 1344 appears to be workwoman whereas uveraine $1298^{\circ}$ seems to have the meaning of work to be done about a place.

oveskaus 1452, 1474 = ecclesiastics; G. only quotes as adjective.

1 This is translated directly from Bede, cap. xvII (xIx), ed. Plummer, p. 246 Unde et a copia anguillurum...nomen accepit. 
seüz $562=$ hunting dogs $\}$.

suchier 339 < suspicari

G. Paris, Introduction to St Gilles

p. xviii, remarks that these two words seem not to be found after the twelfth century.

soil 1476. In the sense this word bears here, G. quotes no clear instances (cf. $\S 20$ ). There is another instance in Sanson de Nantuil (apud Bartsch, Lang. et Litt. col. 152. 35).

tackes 281 = the fastening of the cloak, i.e. fibula, as found in the glossary mentioned in Documents manuscrits (P. Meyer) p. 126 and published in the Jahrbuch für rom. u. eng. Literatur vii 37-8.

teoldé 874. The verb teolder is unknown elsewhere; as stated in the footnote, the word refers to the raising of the awning (tialz) over the waist of a ship when in port, its object was to increase the accommodation. Boats with tilts-tilt-boats-were in use on the Thames in the last century. In line 874 bien should be omitted.

veage 375 appears to have the meaning 'phase'; in this sense it is not quoted by Godefroy.

volage $239=$ a frivolous person; not quoted by $G$. in this sense till 1386. Chardry has the word with the meaning frivolity: Car jeo sai ke par volage, Estes entrez en tele rage. Sept Dormanz 431-2.

a ceo que 1199, 1591. As will have been seen from $\S 53$ viii, this conjunction is used to imply both simultaneous action with and posterior action to that of the principal verb. In the first sense its use is older than in the second and it should be noted that the first occurs in the older portion (O) and the second in (A). Mr Ritchie only found en iceo que in the twelfth century works he examined.

a merveille 215. This is an early use of the locution; generally one finds in Old French merveilles in this sense; this occurs in the same MS. in the life of St Audry, folio $117 \mathrm{v}^{\circ}$ a. Early instances of a merveille and a merveilles are to be found in Roman de Troie 1175, Guillaume de Palerne 36, and the Lai de l'Ombre 557, but merveilles is found as late as the Agn. life of Edward the Confessor (1256) 1. 638.

a peine...que...ne 351. This seems to be the oldest case of this conjunction in the sense of scarcely... when... I have not found it before Froissart: A paines estoient Flamenc cheü, quant pillart venoient... and Chevalier du papegai: il n'ot mie a peine finie sa parole, quant il vit venir ung chevalier (quoted from Tobler, Vermischte Beiträge, iv, p. 40). Notice that in each case que is replaced by quant. The cases of a paine que quoted by Mr Ritchie, p. 27, as e.g. Cant je.tres bien $i$ pens, a paine ke n'en plor; Poème moral $337 \mathrm{~b}$, are not temporal conjunctions. 
aveir cher $415=$ aimer.

aveir en pris $677=$ to delight in.

curant sur ventre 612 , cf. courir ventre à terre.

$n ' i$ en ad 730, cf. modern $i l$ n'en est rien.

est a venir $261=$ devenir. This is perhaps an anglicism, ef. St Richard $\$ 71$, e.g. sui a venir $=j e$ dois venir. Note this instance is in (B).

fere son bon 508, 513= faire son talent.

fort serreit 851 (cf. $\$ 44 \mathrm{~A}$ ) = it would be difficult to... There are analogous cases in G., cf. Forz serreit...

quant est issi $665=$ since it is so.

qui d'un qui d'el 1466 = what with one thing and another.

saver moun $554=$ to learn the truth about; cf. Romania 1909, p. $420,1.4$.

semblant, voir semblant $1378=$ they see no reason for asking; G. does not quote any example of this use.

souventefeiz 481, cf. St Richard $\$ 57$.

tantes feiz 523, cf. St Richard $\$ 57$; no example in Godefroy.

tel hore est $=$ often, cf. G. under eure, iii 672.

tut dis 1415, 1653; this phrase is common in Agn. (less frequent elsewhere); cf. Bestiaire 2696; Marie de France, Bisclavet 318.

\section{Versifiçation.}

$\S 60$. Divisions of the poem.

Certain reasons for stating that our poem as here published is the work of different periods have been set out in the Introduction; this diversity of authorship is rendered much more evident by an analysis of the versification. The oldest part may be designated as $(0)$; the portion dealing with the punishment of Archbishop Richard as (A); and the Modwenna incident as (B).

The approximate division of the 1694 lines (three have been omitted by the scribe) is as follows:

(O) ll. $1-182,387-1416=1212$ (2 ll. omitted in this section),

(A) ll. 1417-1694 =278 (1 line omitted),

(B) $11.183-386$

$=204$.

The length of section (B) must necessarily be somewhat conjectural; 11. 182 and 382 seem by their similarity to suggest the limits of the interpolation, while a few lines before and after these would seem to 
form the 'join.' Doubtless the matter of 11. 376-386 was in the original form of the poem as it is in both the Latin MSS. that are of importance-Bodley and Lansdown; the Fronch lines are as close a translation as is generally the case in work of this kind. Another factor which weighed with me in thinking that section (B) should end with line $\mathbf{3 8 6}$ is that this line is very corrupt and contains ten syllables and although it is not more corrupt than many lines in Agn. poems, yet if we take out the word home ${ }^{1}$ which seems to clash in sense with rei and re-write the line using good twelfth century forms, we get a perfectly correct line:

\section{Al pöestëif rei Syer.}

From this point onwards the lines are as regular as at any part of the poem.

$\S 61$. The couplet.

In his edition (Romania, xxv, pp. 497-541) of the interesting Agn. poem Le Donnei des Amants, G. Paris remarks : 'Notre poète est encore archaïque en ce qu'il est à peu près absolument fidèle à l'ancienne règle de versification exposée par $\mathrm{P}$. Meyer, et d'après laquelle le sens est toujours arrêté au deuxième vers d'un "couplet" et ordinairement au deuxième vers de chaque couplet ${ }^{2}$. Il va même beaucoup plus loin et se rapproche, par la structure de son vers, des formes les plus anciennes du vers octosyllabique en français. Sur les 1242 vers de son poème qui nous ont été conservés, les huit dixièmes environ ont une quatrième syllabe qui porte l'accent tonique, et qui dans plus des deux tiers finit un mot (ou est pénultième avec élision de l'e final), tandisque dans l'autre tiers elle est pénultième sans élision. Des vers restants, la plupart ont une césure féminine avec accent à la quatrième syllabe (Mires diënt que c'est santé), et il n'y a guère qu'une vingtaine de vers en tout qui ne rentrent pas dans l'une ou l'autre de ces formules. Un pareil état de choses ne saurait être fortuit il suffit de mettre en regard

1 In the life of St Audry in the same MS. the same scribe has made the same interpolation.

${ }^{2}$ In Romania, xxII, pp. 1-35, M. Paul Meyer shows by very many examples that except perhaps for Adgar who is not careful of the couplet (it should be noted that, whereas English retains the etymological meaning of couplet, in modern French the word implies tirade or stanza) Agn. poets adhere to the rule of ending the sense with the couplet. He adds that for saints' lives this is generally the rule. The rule is observed too in those of the fourteenth century as e.g. Bozon, cf. Zeitschrift für rom. Phil., xxxIv, p. 295 and in those I have published, ef. footnote 4 of the introduction to St Osith. $M$. Meyer shows that the poet of importance who dared to break this rule is Chrétien de Troyes closely followed by Raoul de Houdenc. I am not aware that any Agn. writer has followed these innovatious to any great extent. 
de notre poème les 1200 premiers vers de Perceval ou de Méraugis pour voir que la proportion est tout autre....'

Considering our poem from the same points of view we find that the rule of the couplet may be said to have been observed almost without exception. Thus ll. 137-8 do not seem to be satisfactorily coupled and ll. 301-4 seem rather to be built up of three lines and one line, although the sense is complete with the fourth line, while ll. 329-331, $1471-3$ and 1474-6 form what are probably the only real infractions; it should be noted, however, that these occur in (B) and in (A) only. Groups in which the sense is complete in two couplets are of course numerous, e.g. 1295-8, 1299-1302, etc. These infractions in (B) and in $(A)$ are one of the many points for ascribing them to a later period than the main body of the poem.

$\S 62$. The line-syllabism.

Añ analysis of each separate line of the poem gives the following results :

A. Metrically correct octosyllabic lines:

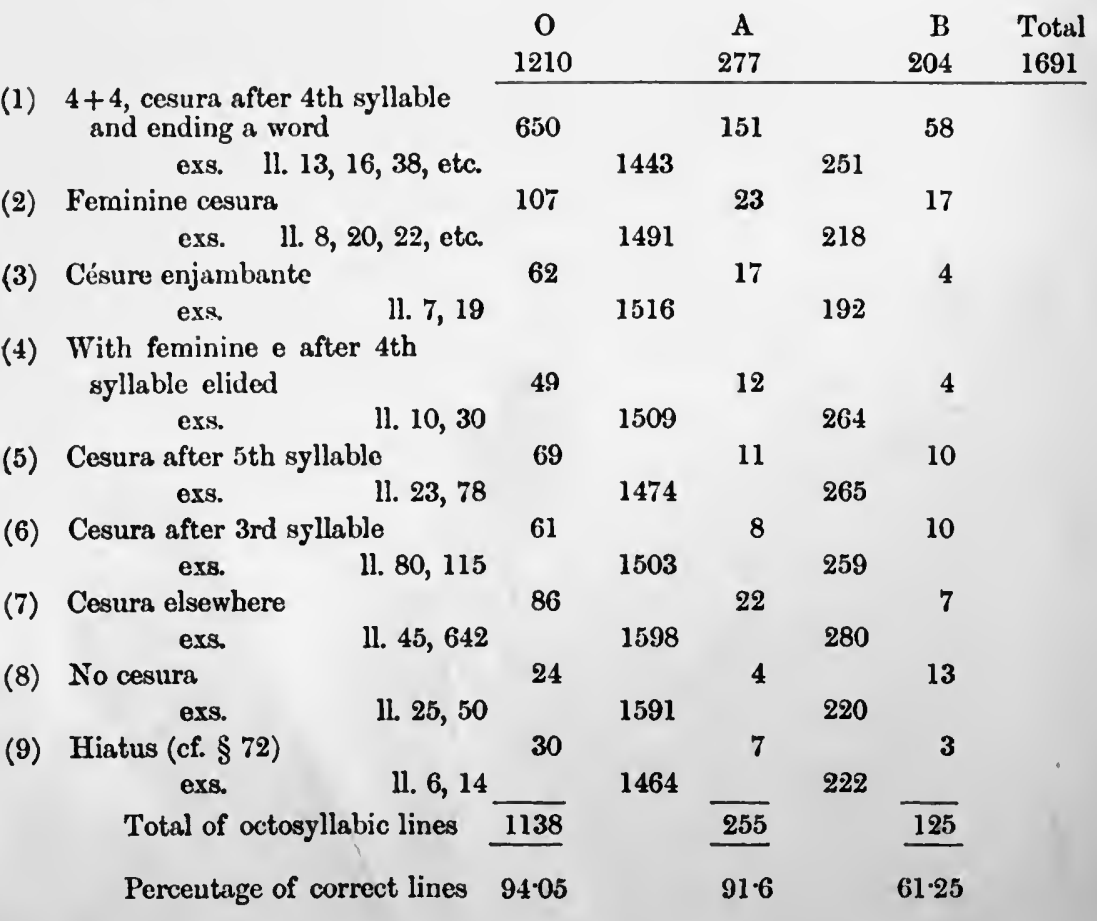


B. Metrically incorrect lines :

(a) Catalectic lines:
(1) 7 syllables $(4+3)$
1I. 32
7
(2) $7(3+4)$
exs.
2
$2 \quad 2$
291
11. 572
$\cdot 1576$
256
(3) 7-no cesura
ll. 808

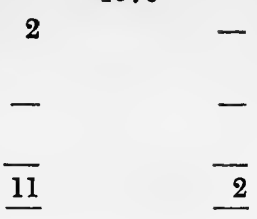
(b) Hypercatalectic lines:
(5) $9=(4 v+4)$ epic cesura exs.
11. 542
7
5
(6) $9=(5+4)$
1580
249
221

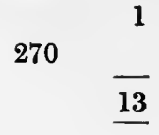
(7) $9=(4+5)$
Il. 898
4
10
232
exs.
ex.
(8) 9-cesura elsewhere exs.
1I. 155
(9) 9-no cesura
exs.
Il. 86
(10) Emendations made in footnotes or $\$ \$ 64-70$
Total of incorrect lines
$\underline{61}$
Percentage of incorrect lines
$5 \cdot 95$

Comparing our results with those obtained for the Donnei, we find that they are almost precisely the same; in that poem two-thirds of eight-tenths (i.e. eight-fifteenths $=664$ lines $=53.3$ per cent.) are divided $4+4$ or else have elided $e$, corresponding to our numbers (1) and (4); taking these for $(O)$ we find our result is $57 \cdot 8$ per cent. and for $(O)$ and (A) together 57.9 and for the whole poem 54.6 per cent. The remaining divisions do not exactly tally with our divisions; G. Paris does not state how he regards lines in his poem like:

(1) Tant poeit munter la parole 297,

(2) Esgardai les pomers floriz 13,

(3) Qï ses oiselès chanter 15,

which would have been classified in our tables under numbers (5), (6) and (7) respectively; but if we add the classes (3), (5), (6), (7) and (8) together we arrive at $24: 9$ per cent. which is practically the equivalent of 
his four-fifteenth (or one third of eight-tenths as he says). That our poem should show an excess of regularity over the Donnei is quite in accord with the soberness of the subject.

$\S 63$. The line-position of the cesura.

The question whether the octosyllabic line has a cesura is much debated; the answer is certainly in the affirmative for a number of the oldest poems as the Pussion, vie de S. Léger, Gormunt et Isembart, Roman de S. Michel, Voyage de Brandan, Roman de Thèbes, and the answer is possibly also the same for the Roman d'Enéas, Roman de 'rrvie and the Romun de Rou and a great deal of the work of Marie de France. In a recent dissertation ${ }^{1}$ the matter has been thoroughly gone into and a large number of texts carefully analysed. It is sometimes a little difficult to agree with the conclusions arrived at; thus e.g. the lai Les dous A manz of Marie de France is stated to have 'einen Einschnitt in der Mitte' in 224 lines out of the 254 , i.e. 88.2 per cent. I have examined this lai on the same plan as our poem (see preceding section) and should divide its lines in the following way: (1) 115, (2) 29, (3) 24, (4) $10,(5) 14,(6) 12,(7) 36$, (8) 13 . Therefore to arrive at the same results as the author of this dissertation we should need to agree that all lines except those under (5) and (6)-where the fifth and third syllable is tonic and ends the word-had an 'Einschnitt in der Mitte': but could the following lines be said to be so divided?

Exs. of (3), 24 in all :

(a) que mult est saive de mescines tant cunuist herbes e racines $107-8$.

(b) tels letuaires vus durra 113.

(c) cungie demande a s'amie 130 .

Exs. of (7), 36 in all :

(a) ne le refreschisse le cors 147 .

(b) ne m'arestereie a nul fuer 200.

(c) unkes si dolente ne fu 232.

Exs. of (8), 13 in all :

(a) car ne s'en poeit consirer 28.

(b) e par druërie l'amast 66 .

I point this out because the author indicates that a percentage of over 80 per cent. of lines with 'Einschnitt' in the middle is necessary

I Melchior, Der Achtsilbler in der altfranzösischen Dichtung mit Ausschluss der Lyrik. Leipzig, 1507. 
before it can be stated that such system is intentional on the part of the poet. I would rather take the stricter view of the octosyllable with cesura and put the dividing line at about 60 per cent. I am inclined to believe that so far as Anglo-Norman saints' lives are concerned a pause is intentional and that it is due to the influence of music which continued to work when such poems were destined only for reading aloud. A lengthy examination $I$ have in hand tends to show that saints' lives differ enormously in this particular, whereas the life of St Audry (end of xII century) has under Nos. 1, 2, 4 (cf. §62 A) only 53 per cent., another life-that of St Andrew (written probably about the same time and by a Frenchman resident in England)-has fully 90 per cent. of these lines. It would seem probable that the skill in 'psalmody' ${ }^{1}$ often attributed to pious people in Agn. saints' lives could extend to skill in reading - and a good deal of skill must have been necessary to make the ill-built lines run at all smoothly-the lives of saints.

An interesting side-light on the part to be left to the reader (or speaker) of Agn. lines is to be found in the Latin directions for the actors in the old drama of Adam²...et, in rithmis, nec sillabam addant nec demant, sed omnes firmiter pronuncient et dicantur seriatim que dicenda sunt.

$\S 64$. The irregularity of the line.

In the preceding section I stated my belief in the existence of the cesura in the Agn. octosyllable. The fact is of prime importance for the discussion of the irregularity of the line. I have stated before that the octosyllabic line is in a vast majority of cases built up of two groups; these groups may be of a varying number of syllables. Further, a feminine $e$, mute in a large number of cases as befits its unimportance in pronunciation, may become sonant when its presence is required to build a group. I will take examples of this phenomenon rather from a faulty thirteenth century text than from this life of St Osith which, so far as regards the main bulk of the poem, is fairly accurate. Thus in the life of St Richard by Peter of Peckham, written about 1270 we find:

(1) after a liquid, feminine $e$ is generally silent:

Par la bele maisun seinte eglise $^{3} 1103$;

1 In the life of St Audry we find: Adammis ont icil a non, Mult ert de grant religion; En vigile et en salmodie, Menoit iceo prudom sa vie, folio 111, verso b. This life is written by a nun, named Marie.

2 Das Adamspiel, hgg. Gross, Halle 19072, Romanische Bibliothek, No. 6.

3 This line, torn from its context, might appear to be a line without cesura, but it is not so. 
but when the tendency to group is evident we have:

De la cure k'avant aveit 788 .

(2) after a dental, feminine $e$ is frequently silent:

Que tute la bible pres oït 796;

but when it is desired to form a grammatical unity or group:

Ot comencié de tute part 174.

Dunc fu preisé de tute gent 506.

Tute l'esglise enluminerent 720 .

(3) after a nasal, feminine $e$ is generally silent:

K'a peine les piez moveir poeit 985 ;

but to form a group:

Est ceste eglise cum lune bele 1118 .

Sachez le veir en bone fei 1337.

(4) the ending -ent (-eient, -erent) is also often silent:

D'ovre de martel furent forgez 688.

- K'il aveient tel tresor perdu 755 .

Jugerent lequel le deust aver 605 ;

but to form a group:

Le loerent ke il amast 267.

(5) between consonants (especially muta cum liquida) feminine $e$ is often silent :

Priveement a sei appella 1331.

Son chapelein k'especial aveit 1321 ;

but to form a group:

Sire Williame sun chapelein 1373.

There is another phenomenon that strengthens my view, viz. that the line that has no cesura is not only rare but comparatively regular in scansion (cf. the tables, $\S 62$ ). Again in cases where the line without cesura is too long or too short it is susceptible to very simple and evident emendation. Taking examples again from St Richard we find:

(6) after a vowel, feminine $e$ is generally mute:

E jeo par l'aïe seint Richard 59;

but when the line has no pause:

Dunt joie de quer de li out 609 .

Dunt jeo esteie tant grevez ${ }^{2} 548$;

(7) after a liquid, feminine $e$ is silent:

De fere dreiture ne se feint mie 636 ;

but when the line has no cesura:

$\mathrm{E}$ issi fere le soleit 781 .

1 This line could be emended to ...itant, but the form only ocsurs once in the poem. 
A sonant feminine $e$ after a de tal, a nasal, in the ending -ent and between consonants (for mute $e$ see Nos. (2), (3), (4), (5) above) is found in the following lines:

(8) E grant multitude de gent 1531.

(9) Veeir coment l'alme passa 1516.

(10) (a) Ke mal me furent par itant 549,

(b) Pur ceo chanterent sanglutant 1684.

(11) L'ercevesque e sun chancelier 714.

When then Professor Suchier says in his memorable review of Atkinson's edition of the Vie de seint Auban (p. 26): 'Sobald der Achtsilbler 'sechs oder neun Silben hat, ist die Cäsur obligatorisch,' I should agree with him, only I should add that because of the cesurc such irregularity becomes possible. I should not like to suggest that the ear of the Agn. writer was absolutely at fault but the words of the defective hemistich must have been so pronounced as to produce an approximation to the necessary four syllables or at least have produced a similar effect. It would be hazardous to attempt to say how nearly or in what way a line containing one or two defective hemistiches was extended in pronunciation so as to approximate to the octosyllable but one or two remarks may not seem beside the point. It will be noticed in the following paragraphs and in the examples quoted by Suchier in the above-mentioned review that most of the defective hemistiches contain a diphthong and that when this diphthong had $i$ as its second component that letter is represented by $y^{1}$. This would certainly seem to be an indication as to the pronunciation to be adopted by the reader. Again that é could be diphthongal is proved by $\S 15$, while the practice of inserting $y$ or $i$ before the tonic when a vowel follows (cf. $§ 15$ ) and the frequent spelling of an accented vowel by double letters must surely point to change in pronunciation.

It seems to me that in this grouping or non-grouping must lie the explanation of the irregularity of the Anglo-Norman line.

$\S 65$. The line with 7 syllables.

A great many of the inaccuracies of an Anglo-French work in a MS. written in England must doubtless be attributed to the scribe, but one and the same scribe cannot be blamed for the difference in inaccuracy such as is found in our poem $-7 \cdot 2$ per cent. for $(\mathrm{O})$ and $(\mathrm{A})$ combined and 38 per cent. for $(\mathrm{B})$ : this must be attributed to the authors.

1 I an, of course, aware of the various criticisms of Suchier's theories, but they amount generally only to emending the exs. used, yet it is admitted by all that this may be carried too far; there is in evéry text a residue of defective lines not susceptible to emendation. 
I have stated in the foregoing paragraph that I ascribe this inaccuracy mainly to the grouping or non-grouping, but firstly all reasonable efforts should be made to secure as accurate a text as possible. First I will consider the short lines in (O) and in (A).

32 This line consists of one correct group and another that has three syllables. I suggest that the second group was originally bor fu cil nez, but here also the word bour with diphthong suggests another explanation.

92 This line is emended in a footnote.

572 This line is defective in its first hemistich; this half line would possibly be read tut a pi-yé, the diphthongisation being rendered possible by the following vowel of alé ; the emendation trestot a (or de) pie is also possible.

808 This line having no cesura should be emended; the emendation is obvious, the Latin text has caput...ambubus manibus; our text should therefore have dous mains.

844 Defective in the second hemistich; the future ert may be read iert diphthongally; it would be possible to emend to sera.

958 Defective in the second hemistich; here the diphthong of vient seems to explain the deficiency. I have noticed a short line again and again in Agn. with this word.

968 As there is no cesura emendation is necessary; I propose to insert tut: De tut ceo....

1034. After the impersonal verb avint a relative ceo should be inserted, cf. l. 322 .

$1186,1254,1336,1385,1405$ are emended in the footnotes.

1576 In the text I have corrected on the grounds that the longer form appears in 1.1623 , it would perhaps be better to allow the form of the MS. to stand and to insert a necessary co-relative ceo before esprover, the line would then fall into the two group system.

In (B) the faulty lines are much more numerous; in cases where the cesural pause is not strongly marked or where there is no cesura I propose to emend as follows:

190 Corr. en fere cert; the phrase requires a complement.

221 Use the form Modwenne as in line 217 and explain hiatus as in $\S 72$.

225 Corr. ceo aveneit, a co-relative is lacking, cf. Erec 5809, Cligès 4440 and line 322.

256 Corr. trestut l'or.

291 Corrected in the text. 
293 Read amedeuz as in line 341 which should become amdeuz.

296 It would seem simple. to emend came en un puiz but the meaning is obscure since it is not clear whether the author means to say that Osith and her book were actually sunk in a hole in the river bed or whether the krenke is a puiz.

298 Corr. en icel duit.

323 Except for the instances in G. I have met the form leus only in the Agn. MS. of St Marie l'Égyptienne; lues mearıs in Old French ' immediately,' but neither in that text nor here does this meaning seem to come out of the context. In St Marie it is emended in various ways by the other MSS.; it seems to me that here the text should read $i l u e c$, and this would then give the required eight syllables.

363 Use the form Modwenne as in line 217.

367 The line may be read with pause after ewe; cf. $\$ 72$.

373 Read adonc.

In making so many emendations in (B) I might seem to be abandoning the principles laid down in $\S 63$, but this is only apparently the case; (B) is a thirteenth century episode and the faulty lines in poems of that century are so by reason of their hypermetric lines rather than by those that are too short; a glance at the tables in $§ 62$ will show this; there the hypermetric lines for $(\mathrm{O})$ are no more than 53 out of 1210 lines while (B) has 69 out of 204; therefore the short line in a thirteenth century poem is much more likely to be due to the scribe than is the hypermetric line'. In St Richard the lines with seven syllables did not amount to five per cent. of the incorrect lines and were readily emendable.

$\S 66$. The lines of 9 syllables with epic cesura.

The existence of this cesura is generally denied for all except a few of the oldest poems, e.g. the Passion :

Jesus reis magnes est sus montez 26.

Chantet aveient de Jesus Crist 28.

Sus en la piedre li angles sist 401.

In other poems it is generally taken as a negligence. In certain Agn. poems however it occurs so frequently that I am inclined to see in it a recognised practice, a natural outcome of the division of the line

1 This phenomenon is nat without exeeptions, e.g. in the life of $\mathrm{St}$ Thomas (ef. $\$ 66$ ) out of 432 lines 163 are short and 21 only too long according to the editor, while in the life of Edward the Confessor (1265) the first 700 lines contain 135 short lines and about 30 of 9 syllables. 
into groups; it seems logical to assume that if the line may have an uncounted syllable at the end it may also have one at the pause in the middle. I have made a few statistics of the frequency of this line in Agn. octosyllabic poems :

Gaimar: Estorie, ll. 1000-2000 3 cases (1l. 1058, 1590, 1887).

Adgar: 'Theophilus legend (No. 17) 2 cases (1l. 62, 1005).

Vie de S. Gilles

no cases.

Adamspiel

Haveloc (11. 1195)

6 cases.

4 cases (11. 78, 294, 388, 788).

In these poems then it would be impossible to consider this as more than negligences on the part of author or scribe, but when we come to poems of the thirteenth century the number of such lines increases greatly; and we find:

Fragments d'une Vie de S. Thomas (circa 1220) 6 cases in 432 lines.

$V$ ie de sainte Modwenne (first 100 quatrains) 15 " 400 ”

Chardry: Petit Plet (circa 1230) ll. 1-400 28 " 400 "

St Richard (circa 1270) 11. 1-400 43 " 400 ”

The cases of this kind of error in $(O)$ seem to be like those of the first group of poems mentioned above; that is, negligences of scribe or poet and susceptible to emendation; thus:

176 The poem seems to me old enough to suggest that ke be omitted (ef. $§ 53$, iii).

542 ariere: for this form read arier.

623 esteient: verbal endings were the first to be disregarded in scansion, especially where the cesural pause was at all marked. This I prefer to regard as a poet's negligence rather than to suggest the omission of tut.

695 This appears to be a faulty line, though tant may be due to the scribe.

696 Here ke may be omitted; deable as a dissyllable is well known and not merely in Agn.

793 saillirent: This might appear to be a case of omission of word ending as in line 623 above, but the line can be emended in two ways; by omission of initial $E$ or by changing the tense to saillent in accordance with the tense of the second line of the couplet; this last commends itself best to me since the line has no cesural pause and according to $\S 64$ the feminine $e$ should be sonant.

1184 The initial si is not required; additions at the beginning of a line are among the usual contributions of scribes. 
The cases in (A) can all be arranged in the one class; they consist in the uncounted $-e$ or $-e s$ after the fourth syllable; such lines are 1473 , $1475,1486,1580,1616$.

The cases in (B) are like those in (A) and prove that for the author the feminine $e$ after the tonic accent may be mute, especially at the middle of the line : cf. 11. 213, 217, 242, 243, 244, 247, 249, 258, 268, 281, $285,300,308,321,333,341,345,358$.

$\S 67$. The line with tonic accent on the fifth syllable.

The four cases in $(\mathrm{O})$-there are none in $(\mathrm{A})$-are doubtless all due to the scribe; the following emendations are offered:

898 avera: the tense seems incorrect, read $a$.

1033 The pronoun $i l$ may be omitted; cf. $\S 48$.

1099, $12+4$ These lines have been corrected in the footnotes.

The instances in (B) are more difficult of treatment; we must assume that the first hemistich containing five syllables must have been so pronounced as to be brought within the group. It is hazardous to do more than suggest how this was done.

215 The locution a merveille is probably due to the scribe, the usual Old French merveilles (cf. $§ 59$ ) read with mute $e$ would reduce the line.

232 Perhaps a shortened form of the name was used.

241 We may assume the pronunciation $a p^{\prime} l a$; cf. $\S 64$ (5).

274 The pronunciation of damisele seems to have been whittled down to damzel (cf. English damsel) and the same form must be assumed for lines 315,329 , while in line 347 where the expression is more formal and the line without pause, we have the normal number of syllables.

\section{Corrected in text.}

313 The feminine $e$ of bele must be mute (cf. $\$ 64(1)$ ); this seems natural in a stock phrase like bele suer.

316 It seems difficult to assume the pronunciation livr' but instances are plentiful in Agn.

$343-4$ It is suggested in footnote to omit ke; cf. $\$ 53$ (iii).

350 This line has probably been altered by the scribe; though the raising of Lazarus is often referred to in Old French literature, e.g. Jhesus lo Lazer suscitet (Passion, 1. 30), the phrase al tierz jur seems rather to refer to Christ's own resurrection; of Lazarus we read: Venit itaque Jesus et invenit eum quattuor dies jam in monumento habentem (Joannis xi, 17). I would propose to read: Ki fu suscitez al tierz jur (For the 
tense in use in Agn. cf. $E$ cum il fu resuscité in the Agn. anonymous translation of the Evangile de Nicodème, line 11, S. A. T.). I would further support this emendation by remarking that in none of the Latin texts of the Life of Modwenna which the author may have used as sources for the account of Osith's immersion is there any reference to Lazarus.

351 This should perhaps be explained as in 1. 232 ; for a peine cf. $§ 59$.

$\S 68$. The line of 9 syllables with accent on the fourth, i.e. with hypermetrical second hemistich.

212 In thirteenth Agn. the definite article is apt to lose its syllabic value, thus the second hemistich will form a group of four.

273, 284 Read $e w^{\prime}$.

374 Old French reine has been influenced in its development by rei, itys best to take this as an instance of its modern form reine with diphthongal $e i$.

380 enseinement: the feminine $e$ is mute as in St Richard $3, \S 64$.

$\$ 69$. The line of 9 syllables without cesura.

According to my remarks in $\$ 64$ these lines should all admit readily of emendation. Note there is only one in $(\mathrm{O})$ and two in $(\mathrm{B})$.

88 The initial $K u r$ is to be omitted as an addition of the scribe.

195 Two solutions are possible (i) that the initial syllable of dementer's is slurred-a frequent occurrence in Agn., cf. Suchier, Seint Auban, p. 34; Simund de Freine, p. liv and St Richard, $\$ 72$. (ii) that the $e$ of vie is silent. Such lines are not uncommon in Agn., cf. St Richard, $\$ 100$. It must have been left to the choice of the reader to decide on the proper pronunciation.

226 The word loinz may be an insertion due to the scribe; in the life of Modwenna written by Geoffrey of Burton we find: Modvenna fecit manere Atheam cum sorore regis (sc. Editha) in uno [sc. monasterio, cf. 11. 218-9], ipsa vero vacans orationibus aliquantis diebus solitaria mansit in altero... Editha...desideravit mittere aliquid ad magistram suam venerabilem Modvennam. Vocavit itaque puellam Osid et dicit ei: vade, inquit, ad dominam...et defer ei ex parte mea volumen istud. The narrative gives us to understand further that the place of Modwenna's retreat was close by: . 
$\$ 70$. The line of 9 syllables with cesura elsewhere.

Like the lines of the preceding paragraph, these should mostly be subject to emendation. We will first consider these in (O) and (A).

155 Read example en prist de chasteté.

528 Read v'ray and cf. $§ 5$.

1049 Omit cum and cf. $\S 53 \mathrm{x}$.

1449 Omit $E$.

1451 Correct manda; cf. $\S 59$.

1496 Omit ke; cf. $§ 53$ i, iii, A.

The following in (B):

262 The feminine $e$ of message is. mute.

266 This is a case of doubtful scansion like line 195 in the preceding paragraph; either omit kar or consider the final $e$ of fere as mute.

268 Read pucel.

272 Read Modwen as suggested in $§ 67$.

290, 344, Corrected in text.

327 Omit ke; cf. $\S 53$ iii.

365-6 Omit $j a$ and for Osith read ele; the name is rendered unnecessary by its appearance in the following line.

384 The enjambement entails a pause and brings about the mutation of the final $e$ of pere.

§ 71. Other irregular lines.

These occur only in (B) and to a much less degree in (A).

$205-6$ The great irregularity of these lines is probably due to the scribe; in the list of Penda's pious descendants we only read of daughters (ll. 151-2) so that possibly the author only wrote:

E uncles de grant poësté

E auntes de grant chasteté.

210 Read nul' maner'.

214. Read Modwen' as suggested in $\S 70$ and abess'.

235 The first hemistich must be read exampl' trova.

247 Read distrin' and el'.

255 Corrected in text.

261 Read v'nir; for other instances cf., authors quoted, $§ 69,1.195$.

276 For comensa read the equivalent se prist; se prendre like comencier may be used without prepositional complement in Agn.

277 Read river'; the line is otherwise faulty. 
286 The first hemistich consists of six syllables; it may have been read as al prendr' s'abaiss'.

301 This line has no cesura and so should be easily emendable: the sense seems to require 'when the dawn of the fourth day came.' I propose to adopt the reading of the MS. for line 269, en la matinée, and read Del quart jur en la matinée.

304 The original may have read achoise or ochoise and the group have been intended to be read achois' saveir; for initial en cf. $\S 6$.

305 Read El quer....

308 The second group would be read par sperital; the word espirit and its compounds are of varying syllabic values in Agn.; cf. Simund de Freine, p. xlix.

328 Omit vus and read verté, a well-known form collateral with verité.

329 The first hemistich must be read V'istes un' damsel'...; cf. $§ 65$.

330 Read La matinée tierz jur fu hier; the article may be readily omitted with expressions of time; cf. 1.334 and Se vus y demoures tier jur li empereres et si traiteur ont pourparlé une grande traison: Robert de Clari, La Prise de Constantinople, xxiii, 16 ; cf. Fredenhagen, Über den Gebrauch des Artikels, p. 17.

333 Read Un' tel pucel'.

372 Since there is no cesura, this line should be emended; it should probably read Kar l'en le tendreit a fols gas.

386 Cf. $§ 60$.

The following two lines are in (A):

1438 The phrase de Chich is probably a gloss inserted into the text by the scribe.

1440 The aset seems to be spurious and may be deleted, it looks like the mental reservation of an interested scribe.

\section{§72. Hiatus.}

The question of hiatus in Old French poetry has received full and careful attention in the vast work of Rydberg on the history of feminine $e$ in French ${ }^{1}$. It is extremely difficult to classify this phenomenon and examples appearing in one class may well find a place in another. The most salient divisions seem to be: A. Logical hiatus, where a final feminine $e$ counts so as to allow the word its full syllabic value and to give it special prominence in the line. B. Metrical; in which the final feminine $e$ must be counted to preserve the measure and the acceptance

1 Gustaf Rydberg, Zur Geschichte des franzözischen ə. Upsala, 1896-1907, p. 1039. 
of which division entails the acceptance of the theory of a cesura. C. Grammatical ; in which a feminine $e$, becoming final by the decay of the dental ending yet remains unelided before a following vowel. D. Analogical ; in which an $e$ after a heavy consonant group retains its syllabic value even before a vowel.

As instances of these we will quote from Rydberg, taking Agn. exs.:

A. Out Rome/en baillie (6 sylls.), Comput, 1923.

$\mathrm{Ki}$ d'Africke/ala requerre (8 sylls.), Tristan (de Thomas), 665.

B. De la nue/eisut s'en sunt (8 sylls.), Brandin, 1667.

C. Ainz que pusse(t)/entrer païs (8 sylls.), Brandan, 616 .

D. Pur espuser l'altre/ Ysolt, Tristan (de Thomas), 307.

Our poem contains examples of all these kinds; the first group may be subdivided as follows:

A. Logical :

(a) Proper names - these may need special prominence;

1. Querondone/est apellée 184.

2. Modwene/en l'un sujurna 221.

(b) A pause in the sense;

1. Et martyre/a chef de tur 64 .

This may be a case for emendation to the form martirie which may occasionally be found in rime with words in -ie.

2. E cele/en fait doil mut grant $\mathbf{1 1 1 6 .}$

3. Ceste overaine/endormir 1344.

(c) With ne, ou, ke, le;

(a) ne: Ne ci ne/ailleurs ja ne pert 6 .

$\mathrm{Ne} /$ en pernent autre rançun 773.

It would perhaps be better to adopt the Agn. form raansun (cf. St Gilles, 2986). For other instances, cf. ll. 676, 1072, 1087, 1163, 1390, 1528, 1681.

( $\beta$ ) ou: Et meindre/ou ses chers amys 1691 (cf. below D).

( $\gamma) k e: \mathrm{Ke} /$ en puissez aveir aïe 124 .

Also the lines 469, 805, 1146, 1327, 1403, 1487.

( $\delta) ~ l e:$ K'apertement le/unt vëu 1064.

(d) Vowel followed by $e$ (et);

Ly reis escute/e entent 549 (cf. below C).

B. Metrical :

1. Ke de l'ewe/Osith n'issi 352.

2. L'ewe/ou Osith fu neée 367 .

Also lines 1201, 1215, 1649. 
C. Grammatical :

Bien li puisse/ estre gradée 410 .

D. Analogical : after a heavy consonant group;

1. Seinte/e dure e preciuse 74 .

2. Et a creindre/e a doter 106 .

Also lines 152, 159, 222, 233, 488, 687, 720, 1079, 1464, 1569.

Modern editors are admitting a greater use of hiatus than formerly. It is certain that it plays a great part in Agn. I have on hand a very lengthy inquiry into the octosyllabic line and its development, and in the 20000 lines I have already examined its use is evidently very extensive. It would be, however, premature to communicate any of the results here.

\section{§ 73. Enjambement.}

The feeling that secures careful observation of the rule of the couplet will make for regularity in the line itself and enjambement will be rare. The shortness of the octosyllabic line renders an overflowing into a successive line necessary occasionally. Thus Chrétien de Troyes and Raoul de Houdene do not shrink from this licence. An enjambement such as the following ${ }^{1}$ savours of flippancy:

Et n'an i a nul, qui n'et un

Baston cornu de corneillier (Yvain, 5514-5);

but others such as

1. Quant dui chevalier sont ansanble

Venu as armes an bataille (Yvain, 1694-5).

2. Chascuns grant piece mal tailliée

En ot; bien en furent peu (Songe d'Enfer ${ }^{2}$ ),

are very common.

Our poem contains the following cases:

In (B), 5 cases :

Et par les gerrons a sei (le) prist

Son mantel ke le livre obli $282-3$.

Also lines 385-6, 399-400.

The enjambement that takes up the whole of the following line is very common in the octosyllable.

1 Quoted by Tobler, Versbau, and Kastner, Versification; I quote it according to the last edition of Yvain.

2 Quoted from Bartsch, Langue et Litterature, col. 244, 1l. 10-20. 
In (A) there are the following 4 cases:

D'iluec purveit queus enverreit

A Chich, kar ces fors mettreit 1471-2.

Also lines 1474-6 (2 cases), 1499-1500.

In the remainder of the poem we have only two cases :

$\mathrm{E}$ pense bien k'en son muster

Als païs doner a(vera) mester $897-8$.

Ele est a seinte Osith par non

Venue par avision 1211-2.

Both of these are of the kind that is normal. In this particular again we see that the deviations from early continental usages occur most frequently in (B), less frequently in (A), and that they are not prominent in $(\mathrm{O})$.

A. T. BAKER.

ShEFFIELD. 

The Modern Language Review is published four times a yoar. in October, Jannary, April and July. The Annual Subscription ie 12s. Bd net post free, payable in advance, single numbers 48. net with the corresponding equivalents in foreign monies, To members of the Modern Language Association, the Roview can be supplied ot the special anoual subscription of 78 . 6 . payable in advance.

Iditorial communications of general kind and contribations relating espocially to German should be addressed to the Editor in chief Prof J, G Robertson, 90, Regent's Park koad London N.W. those dealing with Fnglish should be sent to Mr G. C. Macaulay, Great Sholford, Oanbridge, and those which relate to the Romance languages to Prof H Oelsner, Taylorian Institution Oxford. Booka for reviey should be addressed to the Editors of The Hodern Languge Revieus c/ Mr C. F. Clay, Cambridge University Press, Fetter Lane, London. E. O.

The collaboration of all interested in linguistic and literary research is invited, and contributions are not restricted to the English langugge

Subscription moy be sent to any bookseller or to Mr C. F. Olay. 




$\checkmark$ 


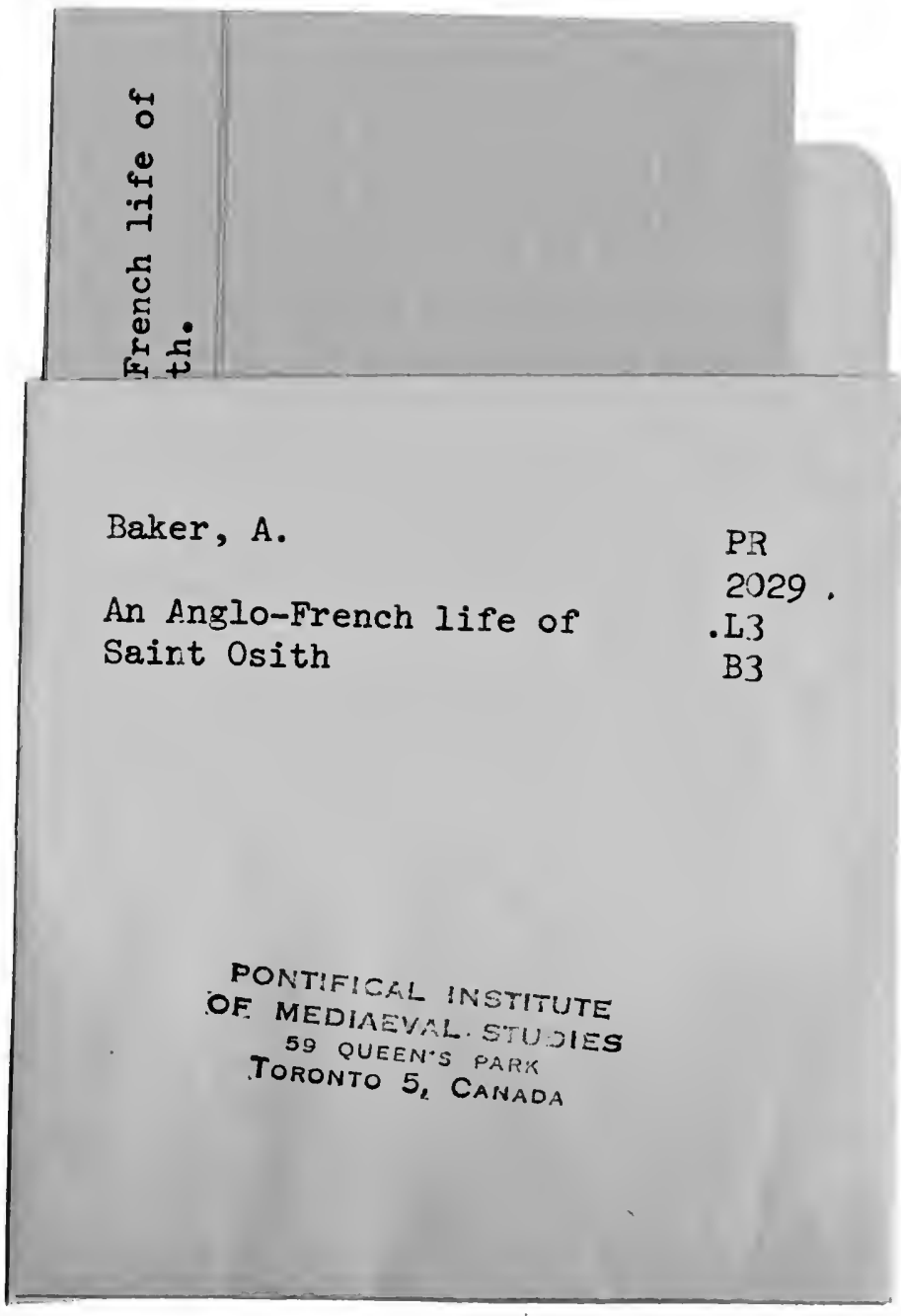


